\title{
La démocratie connectée : ambitions, enjeux, réalité
}

sous la direction de

Michaël Bardin, Marthe Fatin-Rouge Stefanini

Priscilla Jensel-Monge, Caterina Severino

\section{5}




\section{La démocratie connectée : ambitions, enjeux, réalité}

\section{Michaël Bardin, Marthe Fatin-Rouge Stefanini, Priscilla Jensel-Monge et Caterina Severino (dir.)}

DOI : 10.4000/books. dice.5837

Éditeur : DICE Éditions

Lieu d'édition : Aix-en-Provznce

Année d'édition : 2018

Date de mise en ligne : 11 février 2022

Collection : Confluence des droits

EAN électronique : 9791097578046

\section{OpenEdition \\ Books}

https://books.openedition.org

\section{Édition imprimée}

Nombre de pages : 142

\section{Référence électronique}

BARDIN, Michaël (dir.) ; et al. La démocratie connectée : ambitions, enjeux, réalité. Nouvelle édition [en ligne]. Aix-en-Provence : DICE Éditions, 2018 (généré le 09 janvier 2023). Disponible sur Internet : <http://books.openedition.org/dice/5837>. ISBN : 9791097578046. DOl : https://doi.org/10.4000/ books.dice.5837. 


\section{RÉSUMÉS}

Présentant une version enrichie de la Journée décentralisée de l'AFDC organisée à Toulon par le CDPC Jean-Claude Escarras et l'ILF-GERJC, en novembre 2016, cet ouvrage explore l'impact réel de la «révolution numérique» qui modifie, chaque jour un peu plus, nos modes de vie. Cette révolution technologique a une incidence sur la vie démocratique et sur le fonctionnement de nos institutions. Certains assurent déjà qu'internet garantit un meilleur pluralisme de l'information ou expliquent que les blogs, forums et autres réseaux sociaux sont les nouveaux lieux des débats de société. D'autres mettent en avant que ces mêmes réseaux sociaux offrent les moyens aux citoyens d'exercer une sorte de contre-pouvoir. L'Estonie, ou plus récemment la France, ont donné la possibilité aux citoyens de participer à l'écriture de leur Constitution nationale ou au processus législatif via internet. Internet semble ainsi offrir de nouveaux outils à la démocratie. Cependant, cette démocratie connectée n'est-elle pas qu'une illusion? Les représentants sont-ils vraiment plus accessibles? Les citoyens se sentent-ils réellement plus impliqués? Peut-on réellement espérer un renouvellement de la vie démocratique grâce au numérique ? Des risques de dérives semblent poindre. Dès lors, peut-on les éviter ou du moins les anticiper pour mieux les contenir? L'ensemble de ces questions est abordé tout au long de la journée d'étude dont cet ouvrage est issu.

\section{MICHAËL BARDIN (DIR.)}

Maître de conférences à l'Université d'Avignon, membre du LBNC (EA 3788), membre associé du CDPC Jean-Claude Escarras de l'Université de Toulon (UMR-CNRS 7318 DICE).

\section{MARTHE FATIN-ROUGE STEFANINI (DIR.)}

Directrice de recherches au CNRS, Aix-Marseille Univ., Université de Toulon, Univ. Pau \& Pays Adour, CNRS, DICE, ILF-GERJC, Aix-en-Provence, France.

\section{PRISCILLA JENSEL-MONGE (DIR.)}

Maître de conférences, Aix-Marseille Univ., Université de Toulon, Univ. Pau \& Pays Adour, CNRS, DICE, ILF-GERJC, Aix-en-Provence, France.

\section{CATERINA SEVERINO (DIR.)}

Professeur, Aix Marseille Univ, Université de Toulon, Univ Pau \& Pays Adour, CNRS, DICE, CDPC Jean-Claude Escarras, Toulon, France. 


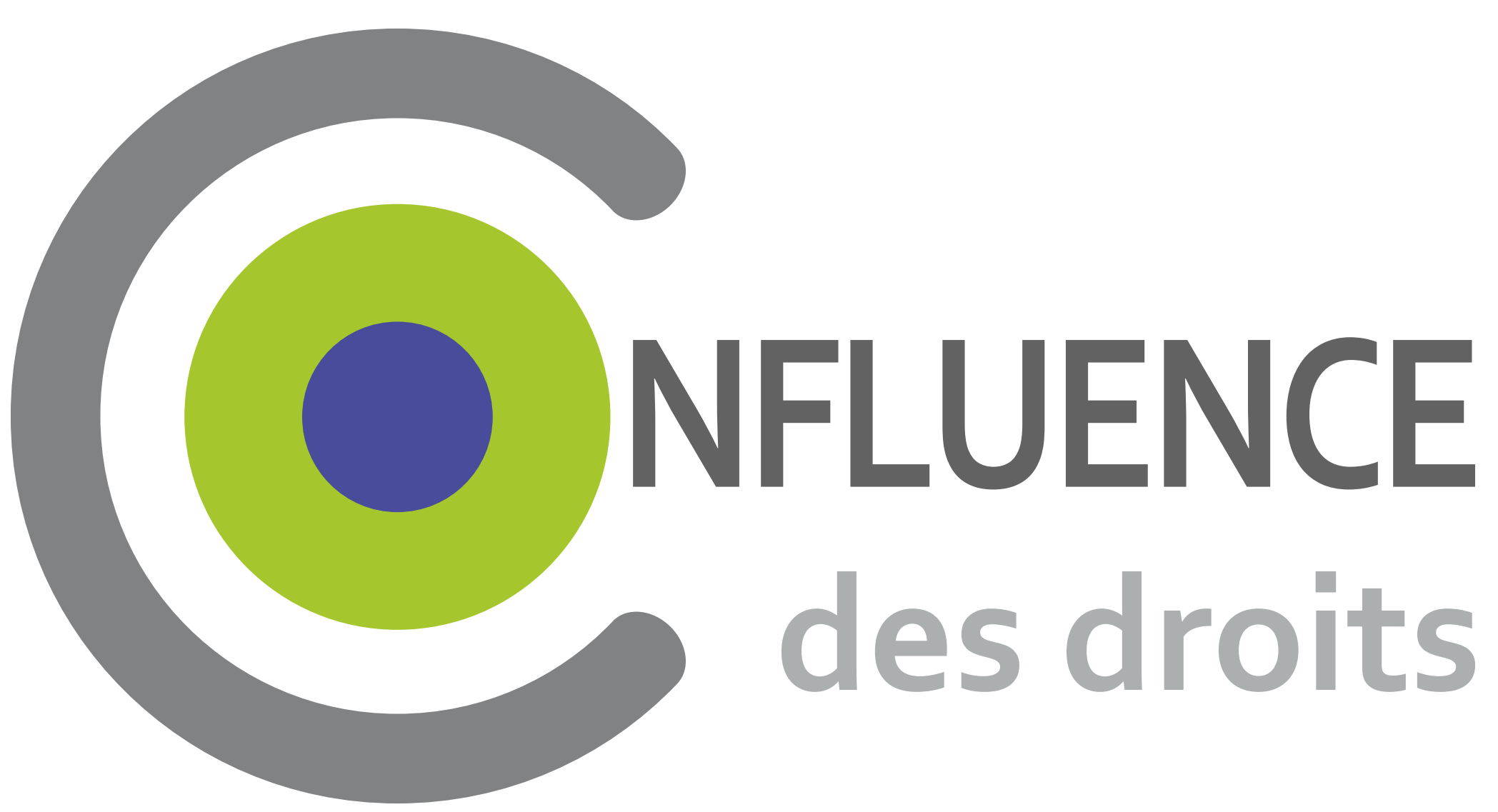

collection d'ouvrages numériques 



\section{NFLUENCE \\ des droits}


MALJEAN-DuboIs Sandrine (dir.), Circulations de normes et réseaux d'acteurs dans la gouvernance internationale de l'environnement, Confluence des droits [en ligne]. Aix-en-Provence : Droits International, Comparé et européen, 2017.

ISBN : 979-10-97578-00-8

FATIN-Rouge STEFANINI Marthe, SEVERINo Caterina (dir.), Le contrôle de constitutionnalité des décisions de justice : Une nouvelle étape après la QPC ?, Confluence des droits [en ligne]. Aixen-Provence : Droits International, Comparé et européen, 2017.

ISBN : 979-10-97578-01-5

RuBıo Nathalie (dir.), La fabrication du droit de l'Union européenne dans le contexte du « Mieux légiférer ", Confluence des droits [en ligne]. Aix-en-Provence : Droits International, Comparé et européen, 2018.

ISBN : 979-10-97578-02-2

TABAU Anne-Sophie (dir.), Quel droit pour l'adaptation des territoires aux changements climatiques ? L'expérience de l'île de La Réunion, Confluence des droits [en ligne].

Aix-en-Provence : Droits International, Comparé et européen, 2018.

ISBN : 979-10-97578-03-9

\section{http://dice.univ-amu.fr/fr/dice/dice/publications/confluence-droits}

ISBN : 979-10-97578-04-6

ISSN : 2556-1162

UMR Droits International, Comparé et Européen (DICE)

Espace René Cassin

3, avenue Robert Schuman

13628 Aix-en-Provence

dice-editions@univ-amu.fr

Références électroniques :

Michaël bardin, Marthe Fatin-Rouge Stefanini, Priscilla Jensel-Monge, Caterina SeVerino (dir.), La démocratie connectée : ambitions, enjeux, réalité, Confluence des droits [en ligne]. Aix-en Provence : Droits International, Comparé et européen, 2018.

Disponible sur Internet : http://dice.univ-amu.fr/fr/dice/dice/publications/confluence-droits ISBN : 979-10-97578-04-6 


\title{
LA DÉMOCRATIE CONNECTÉE : AMBITIONS, ENJEUX, RÉALITÉ
}

\section{ACTES DU COLLOQUE DU 10 NOVEMBRE 2016}

\author{
sous la direction de \\ Michaël Bardin \\ Marthe Fatin-Rouge Stefanini \\ Priscilla Jensel-Monge \\ Caterina Severino
}

Colloque organisé par le CDPC Jean-Claude Escarras (Université de Toulon) et I'Institut Louis Favoreu - GERJC (Aix-Marseille Université) dans le cadre des Journées décentralisées de l'Association Française de Droit Constitutionnel 



\section{SOMMAIRE}

Propos introductifs. Ambitions et limites de la démocratie connectée Caterina Severino, Professeur à l'Université de Toulon

Partie I. Citoyens et numérique : de la communication par internet à l'idée d'une République numérique

Propos introductifs à la séance

Jean Gicquel, Professeur émérite de l’Université Paris I (Panthéon-Sorbonne)

Le citoyen co-législateur : quand, comment, pour quels résultats ?

Ariane Vidal-Naquet, Professeur à l'Université d'Aix-Marseille

Le numérique comme outil de contrôle des gouvernants

Jean-Philippe Derosier, Professeur à l'Université de Lille 2 - Droit \& Santé

Garanties démocratiques et numérique

Sophie Lamouroux, Maître de conférences HDR à l'Université d'Aix-Marseille

Numérique : activisme et influence politique

François-Bernard Huyghe, Directeur de recherche à l'IRIS

Partie II. Gouvernants et numérique : entre véritable opportunité et risque d'instrumentalisation

Propos introductifs à la séance

Pascal Jan, Professeur à l'Institut d'Études Politiques de Bordeaux

Les partis politiques face au numérique : entre adaptation stratégique et émergence de nouveaux mouvements

Michaël Bardin, Maître de conférences à l’Université d'Avignon et des Pays du Vaucluse

L'utilisation des réseaux sociaux par les parlementaires : instrument de revitalisation de la démocratie représentative?

Priscilla Jensel-Monge, Maître de conférences à l'Université d'Aix-Marseille

Les effets des réseaux sociaux dans les campagnes électorales américaines Idris Fassassi, Professeur à l’Université de Picardie Jules Verne

Propos conclusifs. Quel avenir pour le citoyen dans la « démocratie numérique »? Marthe Fatin-Rouge Stefanini, Directrice de recherches au CNRS, UMR 7318 DICE, ILF-GERJC 



\title{
Propos introductifs \\ Ambitions et timites de la démocratie connectée
}

\author{
Caterina SEverino ${ }^{1}$
}

La «révolution numérique » est en marche. C'est ce que l'on nous raconte tous les jours et, il faut dire, c'est ce que l'on constate au quotidien : l'outil numérique a modifié et va sans doute encore bouleverser nos modes de vie.

Mais, quel impact cette révolution technologique peut-elle avoir sur la vie démocratique et même sur le fonctionnement de nos institutions? Certains assurent déjà qu'internet garantit un meilleur pluralisme de l'information. Certains nous expliquent que les blogs, les forums de discussion, les réseaux sociaux sont les nouveaux lieux des débats de société. Certains mettent en avant le fait que ces réseaux sociaux offrent une meilleure visibilité des opinions personnelles de tout un chacun, voire permettraient aux citoyens d'exercer une sorte de contre-pouvoir. Certains pays, comme l'Estonie ou plus récemment la France, ont donné la possibilité aux citoyens de participer au processus législatif via internet, d'autres pays ont, quant à eux, déjà consulté leurs citoyens grâce à l'outil numérique pour qu'ils contribuent à l'écriture de leur Constitution nationale. En France, l'ancien secrétaire d'État au Numérique, Axelle Lemaire, considérait comme un véritable succès le fait d'avoir procédé à une large consultation en ligne des citoyens lors de l'écriture du projet de loi justement intitulé «Loi pour une République numérique $»^{2}$. Aujourd'hui, le Président de la République Emmanuel Macron souhaite, entre autres, le retour du vote électronique pour les Français à l'étranger³, l'outil numérique représentant à ses yeux un levier de «l'exercice démocratique $»^{4}$.

Internet semble ainsi offrir de nouveaux outils à la démocratie.

\footnotetext{
1 Professeur, Aix Marseille Univ, Université de Toulon, Univ Pau \& Pays Adour, CNRS, DICE, CDPC Jean-Claude Escarras, Toulon, France 2 La loi n 2016-1321 du 7 octobre 2016 «pour une République numérique », a été conçue suivant un processus inédit de consultation et de co-création citoyenne, qui a débuté fin 2014 par une concertation nationale sur les enjeux du numérique et s'est poursuivi à l'automne 2015 par une consultation en ligne sur le texte de l'avant-projet de loi.

3 Sur le retour du vote électronique pour les Français résidant à l'étranger, après son introduction en 2012 et sa suppression, pour des raisons de sécurité, en 2017, le Président de la République s'est notamment prononcé lors de la $27^{\mathrm{e}}$ session plénière de l'Assemblée des Français de l'étranger, le 2 octobre 2017 (www.assemblee-afe.fr/allocution-du-president-de-la-republique-emmanuel-macron-a-l-occasion-de-la27\%E1\%B5\%89-session-pleniere-de-l-assemblee-des-francais-de-l-etranger.html).

4 L'outil numérique trouve une place particulière dans le programme présenté lors de la campagne présidentielle par Emmanuel Macron.
} 
Alors, quels bienfaits peut-on en réellement espérer de l'outil numérique dans le renouvellement de la vie démocratique? Et quelles dérives peut-on craindre et donc, si possible, chercher à anticiper? Ce sont les deux questions qui nous accompagnent tout au long de cet ouvrage et qui ont constitué le leitmotiv de la journée d'étude dont il est issu .

Toutefois, avant même d'aborder la question des éventuels bienfaits ou risques de la « révolution numérique » pour la vie démocratique, il nous faut nous interroger sur une question beaucoup plus concrète: quel impact cette révolution numérique a-t-elle vraiment à l'heure actuelle sur nos démocraties?

Car il plane tout de même un doute considérable sur l'incidence que le développement de l'outil numérique peut avoir sur la rénovation de nos institutions : cette incidence n'est-elle pas tout simplement qu'une chimère?

Cette incidence concernerait, visiblement, quatre composantes essentielles de la vie démocratique. Tout d'abord, l'information des citoyens; ensuite, la consultation des citoyens à l'initiative des gouvernants (et donc la participation des citoyens à la prise de décision politique); cette incidence concernerait également la participation spontanée des citoyens à la vie politique de leur pays; enfin, la révolution numérique aurait un impact profond sur les droits et les libertés.

Pour ce qui regarde l'information des citoyens, il est évident que le développement des nouvelles technologies de l'information et de la communication (NTIC) a révélé une méfiance générale de leur part à l'égard des médias traditionnels. Dans ce contexte, d'autres médias, comme Mediapart, ont vu le jour grâce à l'outil numérique. En mettant en avant leur indépendance et leur ambition d'augmenter «l'intensité de la démocratie $»^{6}$, par le biais d'une meilleure information du public, ils semblent indéniablement favoriser le pluralisme de l'information.

De la même manière, le fait qu'internet permette aux citoyens de consulter facilement les médias étrangers, ne peut être considéré que comme un bénéfice au regard de leur possibilité de s’informer.

L'outil numérique offre également une visibilité sans précédent à des organisations comme Wikileaks, dont le but affiché est précisément de réduire l'asymétrie existante entre les informations dont disposent les États et celles dont disposent les citoyens, en donnant la parole et en protégeant les lanceurs d'alerte et en permettant ainsi la diffusion à l'échelle mondiale, d'informations sensibles, comme des cas de corruption, de violation des droits de l'homme ou encore d'espionnage... il est évident que cela bénéficie à l’information de tous.

Le revers de la médaille est connu. Bien des fois, il n'existe aucune garantie concernant la sincérité et la fiabilité de l'énorme flux d'informations qui circulent sur le Web. En France, à l'heure

5 Cette journée d'étude, intitulée «La démocratie connectée: ambitions, enjeux, réalité », qui s'est tenue à la Faculté de Droit de Toulon le 10 novembre 2016, a été organisée par le CDPC-JCE et l'ILF-GERJC, dans le cadre des journées d'étude décentralisées de l'AFDC, édition 2016, portant sur le thème «Le droit constitutionnel à l'épreuve du numérique».

6 Expression utilisée par le co-fondateur de Mediapart, le journaliste Edwy Plenel. 
même où nous écrivons ces lignes, les grands axes d'un prochain texte de loi contre les «fake news», rebaptisé loi sur «la confiance et la fiabilité de l'information», sont présentés par le ministre de la Culture à la presse. L'ambition (vaine?) du Gouvernement est de lutter contre les fausses nouvelles qui circulent sur internet, en encadrant le flux des informations notamment en période électorale et notamment lorsqu'elles proviennent de médias sous influence d'un État étranger. Le texte souhaite aussi introduire une procédure en référé pour permettre aux citoyens de bloquer de fausses informations qui circuleraient sur le Net. Toutefois, ce type d'encadrement législatif ne se fera pas sans heurt, il soulève déjà de nombreuses questions, notamment parmi les journalistes.

Les citoyens étant de plus en plus nombreux à s'informer sur internet, il n'a pas fallu attendre très longtemps pour que les acteurs de la vie politique se mettent, à leur tour, à communiquer par cet instrument. Il est, désormais, inconcevable qu'une femme ou un homme politique ne disposent d'un compte Twitter ou d'un profil Facebook, voire d'un blog. Ces outils donnent la possibilité (ou simplement l'illusion?) de mieux communiquer avec la population, de se montrer plus accessibles, plus ouverts, plus proches des problèmes des citoyens. Par le biais de ces instruments, les gouvernants, ou ceux qui aimeraient bien gouverner, communiquent «en temps réel », commentent tel ou tel événement, telle ou telle affirmation. Ils formulent les points essentiels de leur programme, ils font des promesses, ils s'expliquent, ils se justifient, ils attaquent.

L'outil numérique se présente ainsi comme un instrument susceptible de recoudre la fracture créée entre eux et les citoyens, de reconstruire un dialogue interrompu depuis longtemps.

Mais, pour l'heure, force est de constater que si, d'un point de vue quantitatif, le débat est enrichi, cette nouvelle forme de communication politique, parfois limitée par le nombre de caractères imposé par Tweeter, semble se limiter à la petite phrase et à l'indignation facile. Derrière l'enrichissement apparent du débat, nombreux sont ceux qui craignent, voire dénoncent déjà, son appauvrissement.

Concernant, en deuxième lieu, la consultation des citoyens à l'initiative des gouvernants, l'expérience de certains pays, comme l'Islande ou le Sri Lanka, a révélé qu'elle pouvait être extrêmement ambitieuse. Dans ces pays, les citoyens ont été appelés à contribuer directement à l'écriture de la Constitution par internet. Mais, ces mêmes expériences nous montrent surtout que, pour l'heure, les résultats concrets sont très décevants. En Islande, le texte rédigé à l'aide des contributions des citoyens n'a finalement jamais été validé par le Parlement. Quant au processus sri lankais, il semble actuellement au point mort. Ces deux expériences nous permettent ainsi de constater ce que l'on savait déjà: il ne peut y avoir de consultation des citoyens que si les gouvernants souhaitent réellement les écouter, que si ce type d'initiative ne se réduit pas simplement à un discours de façade. La contribution, dans cet ouvrage, sur le thème de la participation du citoyen à la vie politique par le biais du numérique semble conforter ce constat.

Pour ce qui concerne, en troisième lieu, la participation spontanée des citoyens à la vie politique de leur pays, internet est souvent présenté comme le nouveau lieu du débat politique et comme le lieu idéal permettant aux citoyens d’intervenir dans ce débat. Les opinions échangées dans les forums, 
les débats qui se créent dans les réseaux sociaux, les cyber-pétitions sont autant de moyens offrant à chacun la possibilité d'exprimer son opinion dans ce qui peut ressembler, toutefois, à un vaste défouloir. Ils peuvent permettre d'agréger les gens, de les ressembler autour de certaines idées: internet pouvant ainsi constituer une formidable caisse de résonance de la contestation offrant à l'opinion publique un moyen de faire pression sur les décideurs et même d'exercer sur eux un certain contrôle.

Les contestations populaires qui se sont produites dans de nombreux pays du monde arabe, à partir de 2010, que l'on a désignées par l'expression «printemps arabe», se sont ainsi largement appuyées sur l'outil numérique, certains parlant même de «révolution Facebook», de « révolution Tweeter » ou de «révolution $2.0 »$ tant l'usage des réseaux sociaux et des autres outils d'internet y aurait été important.

On ne peut manquer du reste de relever que l'un des premiers actes d'un gouvernement autoritaire en crise consiste à bloquer les communications informatiques. Tel a été le cas, par exemple, du régime égyptien de Mubarak qui, durant les premiers jours de la révolte, en 2011, demanda l'interruption des communications numériques dans tout le pays; ou encore, de la tentative de coup d'État en Turquie en juillet 2016, l'attaque militaire étant suivie immédiatement par la suspension de Facebook, Twitter et des autres réseaux sociaux, sur ordre du Président Erdogan.

Plus proches de nous et dans un registre différent, il est impossible de ne pas noter que l'éclosion de certains partis, tels les partis pirates en Allemagne ou en Suède, ou Podemos en Espagne, ou le Mouvement Cinq étoiles en Italie, doivent beaucoup à internet.

$\mathrm{Au}$-delà de ces thèmes qui concernent directement la liberté d'information, la liberté d'expression et la participation des citoyens à la prise de décision politique, l'outil numérique pose également d'autres difficultés juridiques majeures relatives aux droits et libertés fondamentaux. Compte tenu de l'importance de cet outil, se pose la question de savoir si l'accès à internet doit être considéré comme un droit pour les citoyens. La loi du 7 octobre 2016 pour une République numérique semble aller en ce sens, en prévoyant le maintien de la connexion à internet en cas de non-paiement des factures, au même titre que pour la fourniture d'énergie et d'eau (article 108 de la loi). Cela reste toutefois, pour l'heure, la seule manifestation de ce qui serait un droit nouveau.

L'outil numérique pose aussi la nécessité de trouver un moyen efficace d'encadrer ses excès. internet est aussi malheureusement le lieu de multiples atteintes à la vie privée, des atteintes parfois très graves. Il peut aussi être le lieu du harcèlement, ou de l'insulte anonyme. Il exige manifestement des réponses juridiques à des questions qu'il pose avec une intensité nouvelle.

Le sujet est donc très vaste et assez complexe, il recèle de multiples aspects, parfois difficiles à saisir de prime abord, mais qui ne manqueront pas d'être mis en lumière par les contributions de cet ouvrage. Les quelques pistes de réflexion ici évoquées ne représentent ainsi qu'un point de départ, laissant une large place à d'autres angles d'attaque. 
Partie I

Citoyens et numérique :

DE LA COMMUNICATION PAR INTERNET À L'IDÉE D'UNE RÉPUBLIQUE NUMÉRIQUE 



\title{
Propos introductifs
}

\author{
Jean GICQUEL ${ }^{1}$
}

De la démocratie connectée ou le passage de la démocratie à la datacratie, tel est l'objet ambitieux de notre colloque. Il faut, à cet égard, saluer l'heureuse initiative des universités d'Aix-Marseille et de Toulon (Instituts Louis Favoreu et Jean-Claude Escarras) et de nos jeunes et talentueux collègues.

Au-delà de la dématérialisation symbolique du «Journal officiel», en application de la loi organique du 22 décembre 2015, le thème de notre colloque bénéficie, sans contredit, de l'actualité. À cet effet, un décret du 10 mars 2017 relatif aux députés élus par les Français de l'étranger autorise «à suspendre le vote par correspondance électronique afin de garantir le secret du vote et la sincérité des scrutins », au vu du niveau de menace extrêmement élevé de cyberattaque relevé par le ministère des Affaires étrangères.

Moyennant des réflexions élémentaires, on conviendra aisément que vivre c'est aller de l'avant; que vivre, c'est adapter, tant pour les personnes que nous sommes, que les institutions qui nous encadrent. Mais, au prix d'une «conciliation équilibrée » entre l'intérêt privé et l'intérêt général; entre «l'exercice des droits et libertés constitutionnellement garantis» et les modes d'action renouvelés de la puissance publique, selon la décision de principe du Conseil constitutionnel (23 juillet 2015, Loi Urvoas sur le renseignement, Rec., p.371).

L'attractivité de la démocratie connectée, celle des citoyens, remet en cause la démocratie représentative, ou le gouvernement du peuple par ses élus. Il est acquis désormais que le processus d'élaboration des normes n'est plus accordé au temps parlementaire et civique. Outre le recours banalisé du pouvoir exécutif à l'habilitation législative (article 38 de la Constitution) les citoyens revendiquent «le droit de concourir personnellement, adverbe révélateur, jusque-là oublié, à la formation de la loi », sur le fondement de l'article 6 de la Déclaration de 1789. L'exemple de réappropriation de la loi du 7 octobre 2016 sur la «République numérique» s’impose naturellement, à la réflexion, avec la mise en œuvre de la consultation de 21000 internautes, parmi lesquels, à n'en pas douter, des représentants des groupes d'intérêts. La beauté du geste évoqué s'en trouve atténuée. Une «loi

1 Professeur émérite de l'Université de Paris-I (Panthéon-Sorbonne). 
corédigée », selon Axelle Lemaire, ministre en charge du projet. Dans cette quête de la découverte de la démocratie directe, s'inscrit par exemple, la proposition de Benoît Hamon, candidat socialiste à l'élection présidentielle, en faveur d'une « 49.3 citoyen », à savoir : $1 \%$ des électeurs inscrits sur les listes électorales pourrait soumettre un projet de loi à un référendum. Dans le même mouvement, le droit de pétition connaît un renouveau. En 2016, plus d'un million de signatures ont été recueillies, par voie électronique, contre le projet de loi Travail.

Au reste, la fonction de contrôle, ce réactif de la démocratie, est repensée dans une mise en perspective avec l'article 15 de la Déclaration de 1789, selon lequel «la société a le droit de demander compte à tout agent public de son administration». Jean-Luc Mélenchon, candidat de la «France insoumise » prône, à cet égard, la révocation populaire de tout élu en cours de mandat, à l'instar du recall américain. De ce point de vue, le rôle des réseaux sociaux bouleverse de fait l'ordre établi. Leur caractère novateur, sinon performant, ne saurait être discuté, à l'instar de Médiapart qui a révélé, en 2013, le scandale Cahuzac et provoqué la réaction du Parlement avec le vote des lois du 13 octobre 2013. Cependant, on est fondé à s'interroger, voire s'inquiéter sur le respect «des garanties fondamentales accordées, aux citoyens pour l'exercice des libertés publiques », au sens de l'article 34 de la Constitution; pour tout dire de «la jouissance paisible de l'indépendance privée », selon l'admirable formule de Benjamin Constant. Qu'en est-il, en effet, de la présomption d'innocence; du principe de la contradiction face à ce tribunal médiatique; du secret de l'instruction? On pense aux affaires Baupin et Fillon? Le procès à armes égales n'est-il pas perdu de vue?

Autant de questions qui vont parcourir notre colloque, à la recherche d'un nouvel équilibre, sous le contrôle du juge constitutionnel, entre liberté et modernité. On peut raisonnablement opiner que sa contribution ne sera pas indifférente au débat d'idées ambiant. «Car, on est toujours apprenti dans la science de la vie», pour emprunter cette maxime à la reine Christine de Suède.

Bon colloque pour chacun d'entre vous! 


\title{
LE CITOYEN CO-LÉGISLATEUR: QUAND, COMMENT, POUR QUELS RÉSULTATS?
}

\author{
Ariane VIDAL-NAQUET ${ }^{1}$
}

La «démocratie connectée», pour reprendre le bel intitulé de ce colloque, offre aux citoyens une nouvelle place dans l'élaboration de la loi. En témoignent les modalités d'adoption de la Loi pour une économie numérique, dite loi Lemaire: après une première consultation «Ambition numérique » menée par le Conseil national du numérique (octobre 2014-février 2015), des contributions citoyennes ont été recueillies (plus de 4000), une «Stratégie numérique » a été présentée par le Gouvernement (18 juin 2015), puis une nouvelle consultation publique sur le texte de l'avant-projet de loi s'en est suivie (septembre 2015-octobre 2015), offrant aux internautes la possibilité de déposer des contributions, celles ayant obtenu le plus de votes ayant la garantie d'obtenir une réponse officielle du Gouvernement ${ }^{2}$. Cette nouvelle façon de faire la loi a permis de rassembler 21000 participants, de réunir 8500 contributions, d'obtenir 150000 votes et a abouti à l'adjonction de cinq nouveaux articles ainsi qu'à la réécriture de nombreuses dispositions ${ }^{3}$ de la loi.

À l'évidence, le recours aux nouvelles technologies de l'information et de la communication (NTIC) permet de développer et de promouvoir la participation citoyenne ${ }^{4}$. L'usage d'internet, notamment, permet d'abolir les barrières spatiales, en supprimant la contrainte géographique, et les barrières temporelles, en favorisant l'immédiateté, de diminuer les contraintes matérielles, en réduisant les coûts d'organisation du vote, de déplacer les barrières sociales, en associant le jeune public notamment. Les citoyens peuvent ainsi participer plus facilement, plus directement, plus immédiatement à la fabrique de la loi. Plus encore, le recours aux NTIC permettrait de renouveler la conception de la démocratie. Alors que la démocratie représentative est en crise, comme le soulignent l'abstention, la défiance des citoyens vis-à-vis des élus, la montée des extrêmes, le succès des partis dits anti-systèmes, la tentation du populisme, l'usage des NTIC promet un regain démocratique, ramenant

1 Professeur, Aix-Marseille Univ., Université de Toulon, Univ. Pau \& Pays Adour, CNRS, DICE, ILF-GERJC, Aix-en-Provence, France.

2 La consultation a été réalisée sur un site dédié, République Numérique, une simple inscription par courriel permettant de commenter les articles, de les modifier ou d'en proposer de nouveaux, de voter sur les propositions des participants.

3 Selon le bilan établi par la société Cap collectif, en charge de la consultation et disponible en ligne (www.republique-numerique.fr/media/ default/0001/02/2377a996950cadd5a093dc9e3a621c0e1f9a19f5.pdf).

4 Ainsi appréhendé, le sujet conduit à exclure les formes de participation citoyenne qui ne présentent pas de lien direct avec les NTIC. Concentré sur l'élaboration de la loi, il exclut la participation au niveau local. 
les citoyens dans l'espace public, leur permettant de participer activement à la prise de décision et rendant enfin tangibles les promesses d'auto-gouvernement que porte en elle la démocratie. Il marquerait l'avènement d'une démocratie participative, d'une démocratie collaborative, voire d'une démocratie «liquide », pour reprendre le titre d'un ouvrage de Baumann, caractérisée par la possibilité pour les citoyens de participer directement à l'adoption des normes ${ }^{5}$. La technique rend possible un renouveau de la démocratie et, plus encore, offre une nouvelle façon de penser la démocratie.

Cette cure de jouvence démocratique promise par le numérique est loin d'être limitée à la France. Pays précurseur, l'Estonie a mis en place, dès l'année 2001, un site participatif appelé TOM (Täna otsustan mina, «Aujourd'hui je décide»), donnant aux citoyens la possibilité de soumettre un projet de loi via un forum électronique, projet soumis à la discussion pendant dix jours sur le forum avant d'être voté par les internautes. Dès lors qu'il obtenait une majorité absolue de votes, il était transmis au ministre compétent qui avait, alors, la charge d'établir un rapport dans un délai d'un mois. Cette initiative a permis d'accueillir près de 2000 idées entre 2001 et 2007, dont sept ont été finalement adoptées, comme l'introduction de l'heure d'été ou la création d'une fondation indépendante pour soutenir les $\mathrm{ONG}^{6}$. Aux États-Unis, un système de pétition en ligne «We the People a a été mis en place par la Maison-Blanche en septembre 2011. Tout citoyen peut, sur cette plateforme, déposer un projet de pétition sur un sujet de son choix, toute demande recueillant 100000 signatures en 30 jours étant assurée d'obtenir une réponse de la Maison-Blanche dans un délai de 60 jours. Autre exemple, celui de la Finlande, avec la possibilité pour les citoyens de proposer des pétitions en ligne sur une plateforme, la loi finlandaise sur les initiatives citoyennes adoptée le $1^{\text {er }}$ mars 2012 assurant à ces pétitions d'être débattues au Parlement si elles réunissent 50000 signatures. Au Royaume-Uni, une pétition peut être déposée sur une plateforme dédiée, obtenant une réponse du Gouvernement si elle atteint le seuil de 10000 signatures et faisant l'objet d'un débat au Parlement si elle atteint celui de 100 000. Au niveau de l'Union européenne a été consacrée, à la suite du Traité de Lisbonne, l'Initiative citoyenne européenne, qui permet à tout projet rassemblant un million de signataires venant d'au moins un quart des pays membres de devenir une «invitation» pour la Commission européenne à présenter une proposition législative sur le sujet...

Toutes ces initiatives concourent à l'avènement d'un citoyen co-législateur, œuvrant avec, aux côtés du législateur traditionnel. Le préfixe implique la participation, l'association, la simultanéité, l'égalité. Plusieurs degrés d'association, rendus possibles par les NTIC, sont envisageables, qui vont du simple partage des informations à la participation au débat et à la discussion ou encore à la décision. Cette «échelle» d'association des citoyens au processus de décision révèle, d'ailleurs, le très grand flottement dans la terminologie utilisée et dans la conceptualisation des notions. Ainsi serait-on tenté de distinguer entre la simple information, qui permet aux citoyens de visualiser, de comprendre ou encore d'assister presque en direct à la fabrique de la $\operatorname{loi}^{7}$, la consultation citoyenne,

Z. Bauman, Liquid Modernity, Polity Press, 2000

Sur l'expérience estonienne, particulièrement riche, voir M. ToOTs, T. KALVET, R. KRIMmER, « Success in eVoting - Success in eDemocracy? The Estonian Paradox », in Electronic Participation, Springer Libri, 2011, p. 55-66.

7 Par exemple, le site «La fabrique de la loi » (www.lafabriquedelaloi.fr/), lancé en juin 2011 par l'association Regards Citoyens et Science Po Paris, se présente comme une plate-forme d'exploration du processus d'écriture de la loi, permettant de suivre le processus, en retraçant 
qui permet de recueillir des avis mais qui peut être vue comme un phénomène passif, sans possibilité d'interaction entre celui qui recueille l'avis et celui qui l'émet ${ }^{8}$, et la participation citoyenne, qui suppose l'implication active des citoyens à l'élaboration d'un acte normatif, en l'espèce la loi, et qui retiendra seule, ici, notre attention. L'association que suppose le préfixe exclut, par ailleurs, les cas dans lesquels le citoyen est seul législateur. À ce titre, ont été écartées de cette étude les opérations référendaires qui évincent totalement le législateur traditionnel, par exemple le référendum d'initiative populaire suisse qui peut conduire à l'adoption directe d'un texte de loi sans passer par la voie parlementaire. Le préfixe «co» renvoie également à l'idée de simultanéité: le citoyen devient législateur en même temps que le législateur traditionnel, ce qui invite à replacer la question au sein du processus législatif: ce dernier a un début, l'idée, le projet, et une fin, le vote de la loi voire son contrôle, le citoyen pouvant intervenir lors de l'une ou plusieurs de ces étapes. Le préfixe renvoie encore à l'idée d'égalité. Le citoyen est-il un législateur comme l'autre législateur? Doivent-ils peuvent-ils intervenir de manière égalitaire? Quelles relations, quels équilibres entre le nouveau législateur et le législateur traditionnel? Cette question s'avère fondamentale, car l'intervention du citoyen reste conçue dans le cadre de la démocratie représentative qu'elle a, précisément, pour objet non seulement de moderniser mais aussi de perfectionner. L'association du citoyen se présente comme un mécanisme correctif, permettant de compenser la crise de représentativité et de légitimité subie par les démocraties modernes. Reste que, en dépit de leur diversité, plus encore de leur hétérogénéité, les expériences de co-législation citoyenne restent pensées dans la logique du régime représentatif, dont elles cherchent, non sans ambiguïtés, à combler les failles. C'est dans cette optique que seront abordées les trois questions posées par les organisateurs: quand, comment, pour quels résultats?

\section{Quand? Le foisonnement des expériences de co-législation citoyenne}

Depuis les années 2010, les expériences participatives faisant intervenir les citoyens dans la fabrication de la loi se multiplient. Ceux-ci peuvent intervenir à des stades très divers, suivant, en cela, la procédure d'élaboration, d'adoption et d'application de la loi: les citoyens peuvent proposer, discuter, voter ou encore évaluer la loi. Chacune de ces étapes est révélatrice du rôle que l'on entend faire jouer à la participation citoyenne.

notamment les amendements proposés et adoptés.

8 Sur la consultation, voir notamment F. Dargent, La consultation en droit public interne, thèse, Aix-Marseille Université, 2015. 


\section{A. L'élaboration de la loi}

L'expérience française témoigne d'une asymétrie très nette entre le traitement de l'initiative de la loi, dont les citoyens français restent exclus, et la discussion de la loi, à laquelle ils sont progressivement associés.

En comparaison des expériences étrangères, l'initiative de la loi reste, en France, très peu développée et très peu renouvelée par les NTIC. Il existe, certes, depuis la Révolution française, un droit de pétition à l'Assemblée nationale ${ }^{9}$. Mais ce dernier est désuet, peu utilisé et peu efficace ${ }^{10}$. Il existe également, depuis la révision constitutionnelle de 2008, un référendum dit d'initiative populaire. Mais ce dernier repose, en réalité, sur une logique exactement inverse, puisqu'il s'agit d'une initiative parlementaire, qui doit être soutenue par un dixième au moins des électeurs inscrits sur les listes électorales, soit environ 4,5 millions d'électeurs, ce qui rend le mécanisme tout à fait illusoire ${ }^{11}$. Tout au plus pourra-t-on relever quelques expériences intéressantes mais fugaces au niveau des partis politiques ${ }^{12}$ ou, plus récemment, sur des sites participatifs ${ }^{13}$, ce qui n'empêche pas que soit réclamée, régulièrement, l'instauration d'un véritable droit d'initiative citoyenne, notamment lors de la dernière campagne présidentielle.

Une fois le texte déposé au Parlement, la participation citoyenne peut se traduire par la possibilité de discuter, d'amender, d'enrichir le texte de loi. La France présente, sur ce point, quelques expériences singulières avec, par exemple, la plate-forme Parlement et Citoyens mise en place depuis février 2013: les parlementaires invitent les internautes à «participer à l'élaboration de la loi », selon la devise du site, en discutant avec eux des propositions de loi, et s'engagent, à l'issue des débats, à présenter à l'Assemblée nationale ou au Sénat la proposition de loi telle que débattue et modifiée ${ }^{14}$. Plus en aval dans le processus législatif, la participation citoyenne peut porter sur un texte de loi déjà déposé sur le bureau d'une assemblée. C'est ainsi qu'à l'Assemblée nationale et à l'initiative de son président, la proposition de loi Clayes Leonetti créant de nouveaux droits en faveur des malades et des personnes en fin de vie a été ouverte aux contributions citoyennes durant plusieurs semaines en $2015^{15}$. C'est dans cette optique qu'a été suggérée l'instauration d'un rapporteur «à la contribution

\footnotetext{
9 Il est régi par l'article 4 de l'ordonnance du 17 novembre 1958 relative au fonctionnement des assemblées parlementaires et par les articles 147 à 151 du Règlement de l'Assemblée nationale, qui en définissent les conditions d'enregistrement et d'examen.

10 Malgré quelques pics sous certaines législatures (jusqu'à 315 pour la VII législature, 1981-1986), le nombre de pétitions décroit régulièrement: 29 lors de la XII législature (2002-2007), 34 depuis 2007.... Ces dernières présentent une très grande hétérogénéité: le plus souvent individuelles, parfois collectives, souvent très générales, parfois précises, demandant tantôt le lancement d'un débat au Parlement, tantôt le dépôt d'un projet de loi ... Les pétitions jugées recevables sont examinées par la commission de lois deux fois par session, cette dernière pouvant prendre trois types de décisions : le classement pur et simple de la pétition, le renvoi de celle-ci à une autre commission ou à un ministre, la soumission de la pétition à l'Assemblée. Deuis 2002, toutes les pétitions présentées à l'Assemblée ont été classées.

11 Article $11, \S 3:$ Un référendum portant sur un objet mentionné au premier alinéa peut être organisé à l'initiative d'un cinquième des membres du Parlement, soutenue par un dixième des électeurs inscrits sur les listes électorales.

12 Ainsi peut-on mentionner le site «Créateur de Possibles», créé par l'UMP en 2009, permettant de rédiger «des initiatives », c'est-à-dire des propositions politiques adressées à un responsable politique, site qui a fermé en 2011.

13 Le site Parlement et Citoyens a mis en place un système de pétition législative en ligne: à partir de 5000 votes, les parlementaires membres de Parlement \& Citoyens s'engagent à répondre à la pétition déposée.

14 À l'heure actuelle, le site regroupe environ une trentaine de parlementaires. Certaines propositions de lois ont connu un certain succès, telle la loi d'interdiction des pesticides à usage non agricole.

15 Rédigées sur Internet, ces dernières ont été répertoriées et analysées avant d'être transmises aux rapporteurs de la proposition de loi.
} 
citoyenne », qui interviendrait aux côtés du rapporteur classique saisi sur le fond ${ }^{16}$ ou encore d'un droit d'amendement citoyen ${ }^{17}$, qui permettrait aux citoyens de déposer des amendements qui seraient présentés, discutés et soumis au vote dès lors qu'ils seraient soutenus par 45000 citoyens.

Si la discussion de la loi est désormais ouverte, pour partie, aux citoyens, l'initiative de la loi reste, en revanche, une prérogative qui leur échappe totalement et qui demeure concentrée entre les mains du Gouvernement, prérogative à peine écornée par la consécration de niches parlementaires.

\section{B. L'adoption de la loi}

L'adoption de la loi échappe encore très largement aux citoyens, ce qui n'empêche pas quelques incursions citoyennes récentes dans l'application de la loi, au stade de son évaluation. De même que la loi reste, pour l'essentiel, initiée par le Gouvernement, elle reste un acte voté par le Parlement.

Curieusement, en effet, les NTIC ne semblent pas avoir révolutionné l'utilisation du référendum comme mode d'adoption de la loi. Pourtant, elles paraissent rendre techniquement possible l'adoption référendaire de la loi, même si l'identification des internautes est difficile. Reste que les critiques généralement émises à l'encontre du référendum, notamment le choix binaire et instantané qu'il implique, perdurent. Si des mécanismes de référendum à choix multiples ont été envisagés, ils ont pour inconvénient d'en relativiser la majorité d'adoption et d'accentuer encore la défiance à son égard. Si une procédure d'adoption d'une loi référendaire article par article a pu être proposée, elle ne permet nullement d'amender le texte, encore moins d'en garantir la cohérence, outre la lourdeur pratique qu'elle susciterait ${ }^{18}$. À cela s'ajoute que la France demeure très réticente à l'utilisation du référendum, qui reste une arme de combat dirigée contre le Parlement et, qui plus est, une arme à double tranchant. Même le référendum dit d'initiative, populaire ou partagé, outre qu'il n'est pas d'initiative populaire, n'est pas non plus véritablement un référendum. En effet, ce n'est que si la proposition de loi n'a pas été examinée par les deux assemblées dans un délai fixé par la loi organique que le Président de la République doit la soumettre au référendum ${ }^{19}$. Autrement dit, le destin ordinaire de cette proposition de loi n'est pas son adoption par le peuple mais bien son adoption par le Parlement, qui conserve, bien évidemment, la faculté de le modifier ou de le rejeter.

L'intervention du citoyen peut encore être envisagée au moment de l'évaluation et du contrôle de la loi, qui semblent être devenues, aujourd'hui, les fonctions principales du Parlement. De ce point de vue, la France présente des expériences intéressantes, qu'il s'agisse de l'évaluation a priori ou a posteriori. Ainsi, en application de l'article 83 du Règlement de l'Assemblée nationale, les citoyens ont désormais la possibilité de s'exprimer sur les études d'impact accompagnant les projets

\footnotetext{
16 Piste évoquée par le vice-président de la commission des lois, D. Raimbourg, chargé en 2015 de rédiger une note au Président de l'Assemblée nationale sur la démocratie.

17 Piste évoquée par le député Olivier Faure et le constitutionnaliste Vito Marinese (https://jean-jaures.org/sites/default/files/notefjj-2671. pdf).

18 C'est par exemple ce qui a été réclamé par Nuit Debout à propos de la loi dite Travail.

19 Selon l'article 11, al. 5, complété par la loi organique 1114 du 6 décembre 2013, si la proposition de loi n’a pas été examinée par les deux assemblées dans un délai fixé par la loi organique (au moins une lecture par chacune des deux assemblées du Parlement dans un délai de six mois), le Président de la République la soumet au référendum (dans un délai de 4 mois).
} 
de loi. À l'heure actuelle, une dizaine d'études d'impact sont ouvertes aux contributions sur le site de l'Assemblée nationale. Quant à l'évaluation a posteriori, elle a été expérimentée pour la première fois en France s'agissant de la loi du 4 août 2014 pour l'égalité réelle entre les femmes et les hommes, les citoyens ayant été appelés, via internet, à donner leur appréciation de l'action publique en faveur de l'égalité autour de 5 thématiques déterminées.

Toutes ces expériences témoignent de la diversité des modalités d'association des citoyens à la loi, les différentes phases pouvant parfaitement s'enchaîner. Et, théoriquement, pourrait être imaginée une initiative citoyenne de la loi émergeant sur internet, reprise dans un projet ou une proposition de loi, ouverte à la délibération citoyenne, adoptées par la voie référendaire et soumise à une évaluation citoyenne. Elle illustrerait une participation citoyenne maximale ou intégrale, de sorte que le citoyen deviendrait purement et simplement législateur à la place du législateur.

\section{Comment? Le fonctionnement de la co-législation citoyenne}

Les expériences de co-législation citoyenne invitent à poser principalement deux questions. La première porte sur les acteurs de la co-législation: qui décide d'associer? qui associe-t-on? La seconde porte sur les modalités de la co-législation: quelle fréquence? quel cadre? Là encore, les réponses à ces questions sont éclairantes sur le rôle que l'on entend faire jouer à la co-législation citoyenne.

\section{A. Les acteurs de la co-législation}

Les expériences menées jusqu'à présent soulignent que la co-législation demeure, pour l'essentiel, un processus piloté par les pouvoirs publics, caractérisant une participation que l'on pourrait qualifier de descendante. Elle est, par nature, tournée vers les internautes, c'est-à-dire vers une fraction seulement de la population, caractérisant une participation relative voire sélective.

Si les NTIC peuvent permettre une participation spontanée des citoyens à la fabrique de la loi, comme en témoigne l'exemple de la loi dite Travail ${ }^{20}$, les expériences de co-législation sont, pour l'essentiel, initiées et pilotées par les pouvoirs publics: elles sont donc le reflet d'une participation dite descendante par opposition à une participation qualifiée d'ascendante ${ }^{21}$. Celle-ci est révélatrice de l'intérêt porté par les pouvoirs publics à ces mécanismes participatifs. Ces derniers permettent de parer la loi de nouvelles vertus, de démocratie certes mais aussi de transparence et d'efficacité, et de légitimer ainsi l'action des pouvoirs publics, avec parfois même le sentiment d'une concurrence entre l'exécutif et le législatif, à qui utilise le plus - ou le mieux - ces procédés. En retour, le pilotage par les pouvoirs publics permet, naturellement, de canaliser ces formes de participation, à défaut de pouvoir les contrôler. On relèvera, d'ailleurs, que la formalisation de ces procédés est, pour l'instant,

\footnotetext{
20 En témoigne la mobilisation très vive à l'encontre de la loi relative au travail, à la modernisation du dialogue social et à la sécurisation des parcours professionnels qui a suscité une pétition en ligne qui a recueilli plus de 1,3 million de signatures et qui a conduit, de facto, à une co-écriture citoyenne de la loi.

21 En ce sens, voir F. Gleize, A. Decourt, «Démocratie participative en Europe », Les cahiers de la Solidarité, nº 8, p. 24.
} 
quasi inexistante en France, en dehors de la contribution citoyenne aux études d'impact, ce qui permet aux pouvoirs publics d'en garder l'entière maîtrise, aussi bien dans leur déclenchement que dans leur fonctionnement.

Ces procédés de co-législation ont naturellement vocation à associer les citoyens. C'est ce qu'illustre nettement l'intitulé de la présente communication et ce qui découle logiquement de l'article 6 de la Déclaration de 1789, selon lequel «La Loi est l'expression de la volonté générale. Tous les Citoyens ont droit de concourir personnellement, ou par leurs Représentants, à sa formation ». Tous les citoyens? Les procédures de participation sont a priori ouvertes à tous les citoyens ou, plus exactement, à tous les citoyens internautes, c'est-à-dire les plus connectés, sans doute plus jeunes, plus urbains, plus qualifiés ${ }^{22}$, de sorte que l'on pourrait se demander si cette co-législation ne serait pas la manifestation d'une nouvelle forme de citoyenneté, une citoyenneté numérique, réservée à certains ${ }^{23}$... Seuls les citoyens? La question est délicate car, abolissant les distances et les frontières, les NTIC permettent voire encouragent la participation des non-citoyens: ainsi les promoteurs de la loi Lemaire peuvent-ils se targuer d'avoir obtenu la participation d'internautes du monde entier, dont il est impossible de vérifier la nationalité, encore moins l'identité. Dans ces conditions, la loi connectée peut-elle encore être considérée comme l'expression de la volonté générale?

\section{B. Les modalités de la co-législation}

Les expériences menées jusqu'à présent soulignent que le recours à la co-législation est à la fois ponctuel et subsidiaire. Autrement dit, la co-législation n'a pas vocation à devenir un mode ordinaire de législation, encore moins un mode intégral de législation.

La co-législation est-elle réservée à certaines périodes, à certaines circonstances ou à certains types de lois? Elle pourrait ainsi être privilégiée pour certaines lois particulièrement importantes, comme les lois constitutionnelles. En témoigne l'exemple très connu de l'Islande, qui a souhaité réviser sa Constitution en 2009 selon une procédure ultra participative en plusieurs étapes ${ }^{24}$. Autre hypothèse, le recours à la co-législation pourrait permettre de légiférer dans des périodes de crise, durant lesquelles les pouvoirs publics manquent de légitimité. Ainsi, l'Estonie a eu recours à la co-législation afin de restaurer la confiance politique à un moment de crise: après des scandales politiques en 2012, a été créée une plate-forme en ligne permettant de consulter les citoyens durant plusieurs mois sur diverses questions, le Chef de l'État s'engageant à transmettre lui-même les propositions des citoyens au Parlement ${ }^{25}$. S'agissant des lois ordinaires, la co-législation peut être

\footnotetext{
22 Voir à ce sujet le bilan établi par la société Cap collectif, en charge de la consultation sur la loi Lemaire.

D’où l'idée que la participation numérique doit être complétée par une forme de participation citoyenne dans des lieux physiques.

Afin d'assurer la révision de la Constitution, un comité constitutionnel mis en place a décidé de l'organisation d'un forum national permettant de consulter directement les citoyens, selon un panel de 950 personnes pour partie tirées au sort. A ensuite été élue une assemblée constitutionnelle, formée de 25 citoyens, élue au suffrage direct et chargée de la rédaction de la Constitution sans interaction avec le Parlement. Durant les travaux de la constituante, il était également possible de participer par Internet, de laisser des commentaires et de faire des propositions. L'initiative a recueilli 3600 commentaires et 370 propositions d'articles, dont la proposition d'établir un droit constitutionnel à Internet, qui a abouti à l'article 14 de la proposition finale.

25 Récoltées sur le site «L'assemblée du peuple (Rahvakogu)», les 15 meilleures initiatives ont été présentées au Parlement. Ce dernier a proposé 7 lois issues de ces propositions ( 3 ont été purement et simplement transformées en propositions lois, 4 ont été modifiées ou complétées
} 
utilisée ponctuellement pour mobiliser les citoyens sur certains sujets considérés comme particulièrement propices ou importants ou particulièrement sensibles. En raison précisément de leur objet, les lois relatives au numérique ont ainsi été ouvertes à la participation citoyenne via les NTIC, en France ou encore au Brésil. Le recours à la co-législation peut également paraître particulièrement pertinent pour les lois présentant de forts enjeux sociétaux (loi sur la fin de vie par exemple) ou dans les domaines souffrant d'un manque de débat citoyen (en matière de santé ou de travail par exemple)... En dehors de ces exemples ponctuels, la co-législation peut-elle être banalisée? Peut-elle devenir un mode ordinaire de législation? Telle est la réflexion en cours en France, le président de l'Assemblée nationale ayant annoncé la création d'un groupe de travail «Démocratie numérique » afin de déterminer comment institutionnaliser la participation numérique et lancer une consultation citoyenne sur la participation des citoyens à l'élaboration de la loi et à l'application de la loi $^{26}$.

Reste que, pensée dans le cadre de la démocratie représentative, la co-législation demeure un procédé d'appoint, qui s'inscrit dans le maintien et le respect des prérogatives du législateur. La démocratie représentative en reste le cadre et la mesure. On relèvera, par exemple, que les initiatives citoyennes demeurent, pour l'essentiel et en dépit de leur appellation, de simples facultés de sollicitation. La distinction est de taille entre une véritable faculté de proposition, c'est-à-dire la possibilité de proposer un texte de loi formalisé qui serait ensuite directement soumis au législateur, et une simple faculté de sollicitation, qui consiste à demander l'inscription d'un texte à l'ordre du jour et qui se présente, alors, comme l'équivalent du droit de pétition ${ }^{27}$. S'agissant des contributions citoyennes, aux projets de loi comme aux propositions de loi, celles-ci demeurent de simples propositions que le Gouvernement, tout comme le Parlement, demeure entièrement libre de rejeter, parfois même sans examen préalable, sauf lorsqu'est assurée une forme de réponse officielle des pouvoirs publics, qui suppose un examen ou, à tout le moins une prise de connaissance minimum de la contribution citoyenne ${ }^{28}$. Quant à la participation à l'évaluation de la loi, celle-ci demeure également très ponctuelle et très faiblement relayée dans le processus normal de l'évaluation et du contrôle. De sorte que, mal valorisée, peu lisible, la participation citoyenne peut sembler peu efficace, simple gadget au service des pouvoirs publics, qui plairait plus par sa nouveauté et son originalité que par son utilité. Plus encore, la co-législation pourrait avoir un effet contre-productif, car l'absence de résultat fait peser, en réalité, un risque de discrédit encore plus grand sur le politique dans son ensemble et une dés-implication plus forte des citoyens.

\footnotetext{
par le Parlement avant d'être votées) et, à ce jour, deux lois ont été adoptées. À ce sujet, voir notamment M. Toots, T. Kalvet, R. KRIMmER, «Success in eVoting - Success in eDemocracy? The Estonian Paradox», op. cit., p. 55-66.

26 Consultation disponible sur le site de l'Assemblée nationale.

27 Il en va tout particulièrement ainsi de l'ICE qui n'est qu'une invitation pour la Commission européenne de présenter une proposition sur le sujet.

28 S'agissant des contributions citoyennes à la loi Léonetti et de leur prise en compte effective par les rapporteurs, on pourra lire dans le rapport de la commission saisie au fond: «La lecture du présent rapport, et notamment des débats en commission, permettra aux internautes de trouver une réponse à nombre de leurs interrogations ».
} 


\section{Pour quels résultats? Le renouvellement de la démocratie représentative?}

En termes quantitatifs, les résultats de la co-législation peuvent sembler décevants. Il en va ainsi à l'étranger des mécanismes de pétition en ligne, finalement peu fructueux ${ }^{29}$. En France, les expériences sont également assez décevantes, malgré quelques motifs de satisfaction, de çà de là̀ ${ }^{30}$. Mais les résultats de la co-législation doivent surtout être appréciés à l'aune des objectifs poursuivis: démocratiser le processus de fabrication de la loi et améliorer la qualité de la loi. Ils doivent également être appréciés à l'aune de leurs effets: de ce point de vue, et paradoxalement, la co-législation tend à accroître les imperfections de la démocratie représentative alors même qu'elle a vocation à les combler.

\section{A. Une démocratisation inachevée}

La volonté de démocratisation est double. Elle se traduit, d'abord, par un élargissement des participants au processus législatif: les citoyens vont pouvoir investir la fabrique de la loi dont ils sont traditionnellement écartés en raison du système représentatif. Elle se traduit, ensuite, par un renouvellement des publics, les expériences de co-législation incitant les générations plus jeunes, et plus familiarisées aux nouvelles technologies, à s'inscrire dans ces processus de débat public. Plus encore se trouve la volonté de redynamiser la société civile. Ces initiatives ont en commun la volonté de placer les citoyens, souvent les plus éloignés du numérique, au centre d'un processus « d'autonomisation» et «d'empowerment». Il y a clairement la volonté de dynamiser le principe représentatif et de ré-impliquer les citoyens dans le fonctionnement démocratique.

De ce point de vue, les résultats peuvent paraître assez décevants et révèlent, surtout, plusieurs biais quasi structurels: une tendance à la concentration de la participation, le nombre global de participants au processus, parfois très élevé, cachant mal une tendance à la monopolisation entre les mains d'une fraction d'entre eux seulement; une tendance à la confiscation de la participation, groupes d'intérêts, associations, syndicats, partis politiques, collectifs participant le plus activement à ces procédés de co-législation, comme en témoigne l'expérience de la loi Leonetti; une tendance à la polarisation négative des opinions, les participants au processus de co-législation étant plus souvent en défaveur du projet ${ }^{31}$, de sorte que la co-législation citoyenne semble favoriser une dimension contre-majoritaire de la démocratie. L'ensemble de ces biais invite à s'interroger, paradoxalement, sur l'identité et, surtout, sur la représentativité de ce citoyen-législateur. Ces procédés qui visent à

29 Aux États-Unis, l'efficacité du mécanisme de pétition en ligne, qui n'existe qu'à la Maison blanche précisément privée du droit d'initiative législative, s'est soldée par l'adoption d'une seule loi en 2014 relative à la possibilité pour les consommateurs d'utiliser le réseau mobile de leur choix; en Finlande, 6 initiatives citoyennes ont été adoptées mais une seule d'entre elles est allée jusqu'à son examen par le Parlement (la loi concernant l'initiative visant à interdire l'élevage de la fourrure) qui l'a finalement rejetée (par 146 voix contre 36); au niveau de l'Union européenne, sur les 51 demandes de lancement d'une ICE, une seule semble s'être traduite par des actions concrètes engagées par la Commission, celle relative au droit à l'eau et l'assainissement.

30 Selon les promoteurs de la loi Lemaire, la participation citoyenne aurait permis d'aboutir à 5 nouveaux articles et à la réécriture de près de la moitié d'entre eux.

31 Cette tendance est, par exemple, confirmée par les rapporteurs de la loi Léonetti: ont participé le plus souvent des personnes qui sont globalement insatisfaites, hostiles ou contestataires. 
remédier à la crise de la démocratie représentative, et notamment à une crise de la représentation, ne conduisent-ils pas à l'association d'un citoyen qui n'est pas, lui-même, représentatif?

\section{B. Une législation imparfaite}

Les processus de co-législation reposent non seulement sur la volonté de donner la parole aux citoyens, mais aussi sur la volonté d'améliorer le contenu des lois. La co-législation citoyenne se présente comme une véritable avancée légistique, permettant d'améliorer tant les qualités formelles que les qualités matérielles de la loi. D’un point de vue formel, elle permet d'accroître la lisibilité et l'accessibilité des lois, désormais écrites par leurs destinataires, tout en raccourcissant - théoriquement tout au moins - les délais d'écriture. D’un point de vue matériel, la co-écriture citoyenne améliore l'efficacité des lois, qui bénéficient de l'expérience voire de l'expertise citoyennes, tout en renforçant leur effectivité, en promettant une meilleure acceptation de ces lois. La diversité des opinions, l'expression du pluralisme, le savoir-faire pratique, la valorisation des réalités de terrain, le recours au bon sens sont autant d'arguments avancés à l'appui de la co-législation. Il y a, au-delà, la valorisation d'une vision procédurale de la démocratie, l'élargissement du débat et des points de vue permettant d'arriver à la bonne décision, mais aussi d'un certain positivisme sociologique, avec l'idée que pourront émerger des propositions de loi ou d'amendements qui répondent le mieux aux aspirations de la société.

En pratique, les résultats peuvent sembler décevants. D’une part, la co-législation n'est pas synonyme d'une meilleure qualité de la loi. Les participations citoyennes semblent passablement pauvres $^{32}$. Plus encore, elles n'échappent pas à une tendance à la démagogie. En effet, la plupart des mécanismes de participation citoyenne ont mis en place des systèmes de sélection des propositions ou des idées ou des amendements qui reposent sur un système de votes, qu'il s'agisse des initiatives de lois ${ }^{33}$, des contributions à l'écriture du texte ${ }^{34}$ ou encore des modifications apportées au texte de loi, encourageant la floraison de propositions farfelues mais clairement clientélistes ${ }^{35}$. Par ailleurs, la co-législation tend à obscurcir clairement le processus de fabrication de la loi. Il existe, en effet, une tendance à la multiplication et à la superposition des mécanismes de participation, comme en témoigne la loi relative à la fin de vie. Cette dernière a donné lieu à un mille-feuille «consultato-participatif », qui altère singulièrement la lisibilité du processus, génère un risque de dilution des responsabilités et contraste avec le fait que le législateur conserve, in fine, le dernier mot $^{36}$.

\footnotetext{
32 À titre d'exemple, la lecture des contributions citoyennes à la loi Léonetti est assez éprouvante, regorgeant de témoignages personnels dotés d'un très faible apport juridique et s'apparentant plutôt à une opération de catharsis généralisée.

33 Qu'il s'agisse de l'initiative de la loi en Finlande (50 000 signature sur 5,5 millions d'habitants, soit environ $1 \%$ ) ou de l'ICE (1 million sur 500 millions d'habitants, soit $0,2 \%$ avec des contraintes de répartition géographique) ou des USA (avec en revanche un seuil très bas de 100000 signatures).

34 C'est le cas de la Loi Lemaire, les contributions ayant reçu le plus de votes des internautes ayant une réponse officielle du Gouvernement et les auteurs des contributions les plus populaires étant reçus par la secrétaire d'État chargée du numérique.

35 Ainsi le site de la Maison Blanche répertorie, parmi les pétitions qui ont reçu le plus de voix, celle de déplacer la fête d’Halloween à un samedi ou de construire une réplique de la station spatiale de Star Wars.

36 Se sont ainsi succédés le rapport de la Commission Sicard, un avis du CCNE, une conférence des citoyens à l'initiative du CCNE, un rapport du CCNE, le rapport de la mission Clayes Leonetti ...
} 
Le respect des prérogatives du législateur et du cadre de la démocratie représentative explique, en réalité, le bilan assez maigre de la co-législation citoyenne. Loin de constituer la panacée, peutêtre même loin du placebo, elle pourrait se transformer en véritable poison, alimentant la défiance, la confusion et le discrédit pesant sur les pouvoirs publics. «Quand, comment, pour quels résultats »; «à quelles conditions»? serait-on tenté de rajouter. La question se pose en termes d'effectivité de la participation (celle-ci doit être représentative, significative et pluraliste), en termes d'efficacité (la participation doit être adaptée, identifiable, traçable avec, par exemple, l'idée d'une «empreinte participative ») mais aussi en termes de qualité du processus participatif (offrant des garanties d'interactivité, de transparence, de lisibilité). C'est à ces conditions que la co-législation citoyenne peut permettre de renforcer effectivement la démocratie. 



\title{
LE NUMÉ RIQUE COMME OUTIL DE CONTRÔLE DES GOUVERNANTS
}

\author{
Jean-Philippe Derosier ${ }^{1}$
}

Sans fin, sans limites, sans frontières. Telles seraient les caractéristiques du numérique comme outil de contrôle des gouvernants. Elles s'appliquent au numérique, d'abord: il permet de voir et d'être présent partout, d'accéder à toutes les informations qui y sont disponibles, sans fin, sans autres limites que les capacités de connexion et de compréhension, au-delà des frontières étatiques traditionnelles. Elles s'attachent au contrôle, ensuite: afin d'éviter abus et tyrannie, il doit être le pendant de tout pouvoir, en s'exerçant de façon permanente et, ainsi mené sans fin, sans limites et sans frontières, il n'en est que renforcé. Elles concernent les gouvernants, enfin: dans une démocratie idéale, ils correspondent exactement aux gouvernés, soit tous les sujets de droit et, si cet idéal est poussé à son paroxysme, la démocratie est elle-même sans fin, sans limites et sans frontières, car elle s'adresse à tous, concerne tout le droit et devient universelle.

Le numérique, le contrôle et les gouvernants nous poussent ainsi à réfléchir sans fin, sans limites et sans frontières. Pourtant, ces caractéristiques ne peuvent être associées au droit, par définition phénomène de limitation ${ }^{2}$. Le droit identifie les gouvernants et limite leurs actions. Il permet, garantit mais aussi encadre le contrôle. Il associe ou non le numérique, en prévoyant ses mécanismes d'intervention, ce qui revient à borner son utilisation. Il impose de limiter l'infini, circonscrire l'universel. Que devient, alors, le numérique comme outil de contrôle des gouvernants?

Ces derniers se distinguent des gouvernés, avec lesquels ils nourrissent toutefois une connexion, d'abord juridique, généralement démocratique, éventuellement numérique. Ils trouvent leur unité dans le peuple, qui peut se diviser en deux catégories: le peuple objet de la norme, c'est-à-dire celui qui en est le destinataire et doit la respecter et le peuple sujet de la norme, c'est-à-dire celui qui l'édicte et qui en est l'auteur. La pure démocratie correspond à une identité parfaite entre ces deux catégories, entre le peuple objet et le peuple sujet, où le destinataire de la norme en est également son auteur'3. Celle-ci ne pouvant être concrétisée, le truchement de la représentation est indispensable. Il conduit à la distinction initiale entre gouvernants et gouvernés.

1 Professeur agrégé des facultés de droit à l'Univ. Lille, EA 4487 - CRDP (ERDP) - Centre « Droits et perspectives du droit (Équipe de recherche en droit public)», F-59 000 Lille, France; Directeur scientifique du ForInCIP et de la revue Jurisdoctoria, auteur du blog La Constitution décodée.

2 En effet, en tant qu' «ordre de la conduite humaine », le droit limite et encadre cette dernière, voire la sanctionne si elle dépasse les limites qu'il pose, c'est-à-dire les normes. Les normes sont donc toutes des limites et il n'est pas possible de considérer qu'en droit positif il existerait quelque chose qui ne soit absolument pas limité. Sur le droit en tant qu'ordre de la conduite humaine, cf. H. KeLSEN, Théorie générale du droit et de l'État, suivi de La doctrine du droit naturel et le positivisme juridique (trad. de Béatrice Laroche et Valérie Faure), Bruylant, LGDJ, Bruxelles, Paris, coll. La pensée juridique, 1997, p. 55 ; H. Kelsen, Théorie pure du droit, op. cit., p. 39-41.

3 Cf. H. Kelsen, La démocratie. Sa nature - Sa valeur (trad. de la $2^{e}$ édition, Charles Eisenmann), Sirey, Paris, 1932 (rééd. Dalloz, 2004 ), p. 14 et s.; cf. également Aristote, Politiques, Livre III, (trad. de Jean Aubonnet), Les Belles Lettres, Paris, 1991, 1283b 43-45: «Un citoyen, d'une façon générale, c'est celui qui participe à la fois aux statuts de gouvernant et de gouverné ». 
Celle-ci opérée, encore faut-il déterminer quelles catégories et institutions les gouvernants recouvrent-ils. Le Président de la République ou aussi le Premier ministre? Le sommet de l'exécutif ou aussi le Gouvernement? Le Gouvernement ou aussi le Parlement? La sphère politique ou aussi l'administration? Le niveau national ou aussi local? Assimilés aux auteurs de la norme, dans la démocratie, les gouvernants correspondent à toute instance de pouvoir (normatif) et c'est bien toute instance de pouvoir qui suppose un contrôle de son exercice. Toutefois, plus un pouvoir est étendu, plus son contrôle est attendu.

Il est ainsi heureux que l'article 24 de la Constitution de la Vépépublique prévoie, désormais expressément, que le contrôle est l'une des missions du Parlement ${ }^{4}$. Il reconnaît ainsi l'une de ses missions traditionnelles et historiques, au même titre que représenter et légiférer, cette dernière étant également mentionnée dans ce même article 24 et l'on ne peut que déplorer que la troisième, pourtant première, ne soit point évoquée. De surcroît, les articles 14 et 15 de la Déclaration des droits de l'homme et du citoyen du 26 août 1789 évoquent aussi la présence et la nécessité d'un contrôle. D'une part, à l'égard de la «contribution publique», dont «les citoyens ont le droit [...] de suivre l'emploi » ${ }^{5}$. D'autre part, à l'égard de l'administration en général, dont «la société a le droit de demander compte à tout agent public $»^{6}$. En plagiant Portalis, qui rappelait que la justice est la première dette de la souveraineté, et en reprenant Guy Carcassonne, on constate ainsi que les Révolutionnaires de 1789 érigeaient déjà la responsabilité et le contrôle qui en découle comme première dette du pouvoir?

Une démocratie majoritaire et moderne impose ainsi un contrôle des gouvernants, réorganisé à partir de la reconfiguration de la séparation des pouvoirs, qui n'existe plus tant entre le législatif et l'exécutif, qu'entre la majorité et l'opposition : à la majorité de conduire la politique du gouvernement, à l'opposition de contrôler la politique ainsi menée ${ }^{8}$ et, le cas échéant, d'alerter ou d'initier les mécanismes dont elle dispose. Cela n'est rendu pleinement effectif que si des droits lui sont reconnus car, minoritaire par définition, elle ne peut prendre aucune décision par un vote supposant une majorité 9 . Ces droits sont alors des « outils» de contrôle, sans toutefois épuiser totalement cette dernière catégorie.

Car le «contrôle des gouvernants » dont il est ici question soulève une ambiguïé : s'agit-il du contrôle sur ou du contrôle par les gouvernants? Le premier connaît un fondement ancien, dans la Déclaration de 1789, qui permet aux citoyens et à la société de contrôler l'administration. Le second est aujourd'hui reconnu à l'article 24 de la Constitution. Ce serait un tort de ne se concentrer que sur l'un des aspects, car des acteurs essentiels de la démocratie, les représentants ou les citoyens,

Article 24, al. $1 \mathrm{CF}$ : «Le Parlement vote la loi. Il contrôle l'action du Gouvernement. Il évalue les politiques publiques ».

Article 14 DDHC.

Article 15 DDHC

G. Carcassonne, La Constitution, introduite et commentée, Seuil, Paris, 11 édition, 2013, p. 25.

Cf. J.-P. CAмBy, «Séparation des pouvoirs, typologie des régimes. Actualité des “régimes parlementaires contemporains" » et C. Vintzel, «La démocratie immédiate et la trilogie des pouvoirs », in J.-Ph. Derosier et G. SACriste (dir.), L'État, le Droit, le Politique. Mélanges en l'honneur de Jean-Claude Colliard, Dalloz, Paris, 2014, p. 111 et s. et p. 123 et s.

9 Sur la question de l'opposition et de ses droits, que ce soit en France ou dans d'autres pays, on renvoie à J.-Ph. DEROsIER (dir.), L'opposition politique, LexisNexis, Paris, Cahiers du ForInCIP, nº 1, 2016. 
seraient alors exclus. Au contraire, en entendant le contrôle des gouvernants comme un contrôle sur et par les gouvernants, on constatera que le numérique permet précisément de renforcer la connexion entre eux: entre les différents types de contrôles et entre les gouvernants et les gouvernés.

Le numérique devient alors un outil du contrôle en revêtant une double dimension. Il ouvre d'une part un mouvement descendant de diffusion, où les gouvernants informent sur leur objet de gouvernement. D'autre part, il permet un mouvement ascendant d'enquête, dont les citoyens se saisissent directement afin de renouveler le contrôle à l'égard des gouvernants. Par la diffusion numérique, les gouvernants s'ouvrent au contrôle, quoique modérément (I) et, grâce à l'enquête facilitée par le numérique, les citoyens font subir un contrôle aux premiers et, cela, assurément (II).

\section{S'ouvrir modérément au contrôle}

Le numérique est un outil de diffusion contrôlée. Il permet aux gouvernants de rendre compte de leur action (A) et de contrôler l'information diffusée puisqu'ils conservent l'initiative de cette diffusion (B).

\section{A. Un outil de diffusion}

La diffusion est facilitée par le numérique (1) et vient renforcer la transparence démocratique (2).

1. Le numérique est d'abord un outil de diffusion d'information, qu'elle soit institutionnelle, à partir de sites des institutions étatiques, ou non institutionnelle, à partir de sites relevant d'initiatives privées (associations, pages personnelles des gouvernants, etc.). Cela renforce la transparence et l'accès à l'information grâce à la rapidité, voire la quasi-immédiateté de la diffusion. Les sites des institutions politiques constituent une plateforme de leur communication. Il est ainsi possible de visualiser la séance publique (de l'Assemblée nationale et du Sénat) en direct, d'avoir accès au compte rendu des débats quelques heures après leur tenue, de lire les décisions du Conseil constitutionnel ou du Conseil d'État sans attendre qu'il en soit rendu compte dans la presse. L'accès à l'ensemble du droit, des débats publics et politiques devient direct et immédiat et l'individu (pas seulement le citoyen) peut suivre l'action des gouvernants, où qu'il se trouve, pourvu qu'il ait une connexion internet (par exemple via son smartphone) et pourvu qu'il comprenne la teneur des débats. De même, les gouvernants peuvent spontanément et immédiatement communiquer sur leur travail, leur permettant d'en rendre compte et d'en répondre. Si le contact humain demeure irremplaçable, il est complété et renforcé par une présence sur internet: la poignée de main sur le marché est toujours appréciée et attendue, mais l'accès direct à l'information est également recherché et exploité par les électeurs.

Ceci vire parfois à la spontanéité, grâce aux forums et réseaux sociaux. Twitter ou autre Facebook constituent des lieux d'expression des personnalités politiques et publiques, voire 
des lieux d'interaction en ce qu'ils permettent un échange direct avec le citoyen. Aujourd'hui, le «Tweet» branché a pratiquement remplacé le communiqué de presse ringard, les personnalités préférant communiquer directement par ce nouveau média, ou d'autres encore comme Snapchat ou Youtube. La période électorale récente, 2016 aux États-Unis, 2017 en France, est éloquente. Convaincre l'électeur ne passait plus tant par des meetings politiques sur des thèmes choisis ou autres confrontations télévisuelles, que par des tweets intempestifs et parfois provocateurs (Donald Trump), des meetings simultanés aux hologrammes démultipliés (JeanLuc Mélenchon), des présences sur Youtube (Jean-Luc Mélenchon encore), ou autres confrontations directes avec les électeurs grâce à Snapchat (Emmanuel Macron). Les journalistes relaient alors, à leur tour, les informations mais le peuple peut avoir un accès direct à la source primaire, lui permettant même, le cas échéant, de réagir, de commenter, de contredire.

2. Cette communication spontanée corrobore une transparence renforcée : si l'on communique, c'est que l'on peut tout dire et que l'on se sent irréprochable dans ses actes et son propos. Cela vaut naturellement pour la communication institutionnelle, qui renforce ainsi la transparence démocratique, mais aussi pour la communication personnelle, qui permet au gouvernant de s'ouvrir aux gouvernés, au responsable politique de s'exposer à ses électeurs. Ceci renforce indéniablement la démocratie en rapprochant l'électeur de son élu et réciproquement: le citoyen-spectateur de la vie publique se transforme peu à peu en en citoyen-acteur dans la vie publique, pouvant librement s'exprimer, dire ce qu'il pense d'une action de son élu ou de son action en général. Le lien entre peuple objet et peuple sujet de la norme est raccourci.

Le moindre secret devient alors suspicieux et démocratie toujours plus exemplaire devient synonyme de démocratie toujours plus transparente ${ }^{10}$. Avant même les lois sur la transparence $^{11}$, consécutives à la célèbre «affaire Cahuzac», les institutions s'ouvraient déjà de plus en plus, le huis clos tendait à disparaître car il se transformait en tabou, les commissions parlementaires siégeant d'ailleurs publiquement autant que possible. Ainsi, autrefois, les travaux de ces dernières n'étaient pas ouverts au public, sauf exception. Au lendemain de la révision constitutionnelle du 23 juillet 2008, le règlement de l'Assemblée nationale a été modifié afin de leur permettre d'organiser elles-mêmes la publicité de leurs travaux, tout en prévoyant qu'un compte rendu des débats serait dressé. Poursuivant dans une volonté de transparence renforcée, les députés ont à nouveau modifié le règlement en 2014 et l'article 46, alinéa $1^{\text {er }}$ dispose désormais que «les travaux des commissions sont publics», réservant toutefois la possibilité pour le bureau de chacune d'entre elles de déroger à ce principe ${ }^{12}$. La publicité est désormais la règle, le huis clos l'exception. Le Sénat, en revanche, maintient le principe du secret des

10 Sur la préservation du secret au sein de notre État démocratique, cf. J.-M. BELORGEY, «L'État entre transparence et secret», Pouvoirs, ${ }^{\circ} 97$ (Transparence et secret, 2001), p. 25-32; cf. également J.-D. BREDIN, «Secret, transparence et démocratie », in ibidem, p. 5-15.

11 Loi organique $\mathrm{n}^{\circ} 2013-906$ du 11 octobre 2013 relative à la transparence de la vie publique, JORF du 12 octobre 2013 page 16824 et Loi $\mathrm{n}^{\circ}$ 2013-907 du 11 octobre 2013 relative à la transparence de la vie publique, JORF du 12 octobre 2013 page 16829.

12 Art. 46, al. 2 RAN. 
travaux des commissions, tout en permettant à ces dernières de décider de leur ouverture au public. Un procès-verbal à caractère confidentiel est dressé ${ }^{13}$.

Contrairement aux idées reçues, le secret d'autrefois n'avait rien de contraire à la démocratie et la transparence d'aujourd'hui ne la rend ni plus efficiente ni plus efficace. Au contraire. Préservés des regards, les députés échappaient à toute pression, médiatique, politique ou publique; ils pouvaient s'adonner en toute tranquillité à des négociations avec le camp adverse permettant de construire un compromis satisfaisant pour tous ${ }^{14}$. Si tel n'était pas systématiquement le cas, loin s'en faut, cela a pu arriver régulièrement. Ce n'était contraire ni aux principes démocratiques ni même à l'exigence de transparence.

La diffusion de ces informations grâce au numérique renforce le caractère transparent de la démocratie. Mais elle est assurée par les institutions elles-mêmes, si bien qu'elle demeure contrôlée.

\section{B. Une diffusion contrôlée}

À l'instar de toute connexion, la démocratie connectée a son corollaire : la démocratie déconnectée. Il y a ainsi une gradation dans la diffusion de l'information, de la diffusion retenue (1) à la diffusion totale (2).

1. Les gouvernants décidant eux-mêmes de la connexion peuvent décider de l'interrompre, de la suspendre ou de la retarder ce qui signifie, plus concrètement, que ce sont eux qui choisissent l'information diffusée, de son instant et de sa méthode de diffusion. Cette information peut ainsi être structurée et organisée, ou, au contraire, livrée en bloc. Aux citoyens, ensuite, de s'y retrouver, ce qui n'est pas toujours aisé. Si bien que la diffusion de l'information effectuée au niveau institutionnel par les gouvernants suppose d'abord qu'elle soit choisie, ensuite qu'elle soit publiée, éventuellement qu'elle soit structurée.

Le choix, la publication et la structuration de l'information diffusée peuvent être généraux ou partiels. Généraux, ils s'appliqueront à tout et la transparence sera totale, sans filtrage ni contrôle. Partiels, ils ne concerneront que certaines informations et la diffusion sera alors effectivement contrôlée. Ce qui ne signifie pas pour autant qu'elle sera altérée, car une diffusion choisie peut conduire à un contrôle ciblé. Néanmoins, le choix entre les deux alternatives demeure entre les mains de ceux qui sont supposés s'exposer au contrôle: les gouvernants qui diffusent l'information ou l'institution à laquelle ils appartiennent. À moins qu'une institution puisse diffuser des informations concernant une autre institution, selon le jeu logique de la

13 Art. 16, al. 5 à 8 RS.

14 Sur le secret comme gage d'efficacité, on renvoie à la négociation et l'accord relatif à la Nouvelle-Calédonie, en 1988, relatés par Guy Carcassonne, «Le trouble de la transparence », Pouvoirs, ${ }^{\circ} 97$ (Transparence et secret, 2001), p. 19-20, ainsi qu'à la conclusion des accords d'Oslo, en 1993, entre Israéliens et Palestiniens. 
séparation et du contrôle du pouvoir, où, selon la formule de Montesquieu, «le pouvoir arrête le pouvoir».

D'abord, il y a des lieux qui demeurent totalement déconnectés, presque au sens propre du terme. Sans évoquer le classique «secret de la défense nationale » ${ }^{15}$, l'un des lieux les plus déconnectés est aussi l'un des plus hauts de la République et des plus essentiels au fonctionnement de l'État: le Conseil des ministres. En son sein, tout y est secret, rien n'y est retranscrit, sauf le compte rendu officiel, dressé par le secrétaire général de la Présidence de la République ${ }^{16}$. Il en va de même de la conférence des présidents ou du bureau des deux assemblées. Si le premier a un rôle essentiellement procédural, en se bornant à fixer l'ordre du jour, on pourrait néanmoins y découvrir des tractations politiques intéressantes, entre le gouvernement et le groupe majoritaire ou entre la majorité et l'opposition.

Ensuite, alors que la volonté de transparence des acteurs institutionnels a été poussée à son paroxysme au lendemain de «l'affaire Cahuzac», par les lois qui ont créé la Haute autorité sur la transparence de la vie publique (HATVP), elle n'est pas allée jusqu'à une transparence électronique totale. Certaines informations ne sont consultables qu'en préfecture et non sur internet (situation patrimoniale $)^{17}$. D'autres sont interdites de toute forme de publication, grâce au contrôle du Conseil constitutionnel ${ }^{18}$. C'est heureux. Espérons que «l'affaire Fillon», qui a agité la campagne présidentielle de 2017, ne débouchera pas sur une nouvelle frénésie de la transparence, imposant désormais aux parlementaires d'indiquer quel(s) collaborateur(s) ils mettent dans leur lit ou, pis, lesquels n’y ont point accès. La démocratie n'a jamais demandé à être impudente.

Enfin, comme son nom l'indique, la «séance publique» du Parlement, en vertu de l'article 33 de la Constitution, impose la publicité: tout ce qui s'y passe, tout ce que les gouvernants y disent est capté par image, par son et par sténographie, pour être retranscrit dans un procès-verbal, désormais accessible en ligne peu de temps après la séance elle-même. C'est la logique même de la démocratie représentative où le titulaire d'un mandat peut certes agir librement ${ }^{19}$, mais ne peut agir secrètement, sans répondre à tout moment de son action face à ses électeurs. Là où la loi est votée et le contrôle exercé au nom du peuple, ils le sont devant le peuple, ce dernier pouvant à tout moment avoir accès aux débats et paroles prononcées

15 Quoique les documents «classifiés»soient ainsi inaccessibles, ils n'échappent pas pour autant à tout contrôle: ils peuvent soit être déclassifés, soit être contrôlés par des personnes habilitées, soit être rendus accessibles au terme d'un certain délai. Sur le secret-défense, cf. B. Grasset, «Secrets défense», Pouvoirs n 97 (Transparence et secret, 2001), p. 63-66 et M. GuILlaume, «Parlement et Secret(s) », in ibidem, p. 67-84; cf. également, sur l'aspect particulier du numérique, B. WARUSFEL, «Secret de défense, sécurité nationale et nouvelles technologies », in C. Blaizot-HAZARD, Les NTIC face aux droits et libertés fondamentaux à travers le prisme du secret, Institut universitaire Varenne, Paris, coll. «Colloques et essais», 2016, p. 33-44.

16 Sur le Conseil des ministres en général et cette question en particulier, cf. Bérengère Bonte, Dans le secret du Conseil des ministres. Enquête dans les coulisses du salon Murat, du général de Gaulle à Nicolas Sarkozy, Éditions du Moment, Paris, 2011.

17 L'article LO 135-2 du code électoral prévoit que, pour les parlementaires, «les déclarations de situation patrimoniale sont, aux seules fins de consultation, tenues à la disposition des électeurs inscrits sur les listes électorales », notamment en préfecture.

18 Le Conseil constitutionnel a censuré ou restreint la portée de certaines dispositions de la loi sur la transparence de la vie publique, notamment à l'égard des élus locaux (dont la déclaration patrimoniale n'a pas à être rendue accessible) et des personnalités non élues (dont la déclaration d'intérêt n’a pas à être rendue publique), décision n²013-676 DC du 9 octobre 2013, Loi relative à la transparence de la vie publique, Rec., p. 972.

19 Selon l'article 27 de la Constitution, «tout mandat impératif est nul». 
par ses représentants ${ }^{20}$. Mais des circonstances contingentes (comme la sécurité nationale) peuvent imposer des travaux en comité secret, permis par la Constitution mais seulement pour un motif déterminé et à la condition que cela soit décidé par l'assemblée concernée ellemême. C'est extrêmement rare et espérons que cela le demeure: à l'exception de la triste parenthèse de Vichy où le Parlement ne s'est pas réuni, les dernières réunions en comité secret remontent à la III ${ }^{e}$ République, pendant la Première Guerre mondiale, et pour la dernière fois le 17 octobre 1917. Le secret, toutefois, demeure transparent et nullement opaque: le comité secret est décidé selon une procédure transparente et établie, un procès-verbal est dressé et dévoilé le moment venu ${ }^{21}$.

2. Au-delà, lorsque la diffusion est totalement ouverte, ou presque, la structuration de l'information diffusée favorise, quant à elle, sa lecture. Au nom d'une démocratie nécessairement transparente, le vote des parlementaires est public (à l'exception des nominations personnelles ${ }^{22}$ ), puisqu'ils votent au nom du peuple et que ce dernier est en droit de connaître les opinions émises et les décisions prises en son nom. Le résultat d'un scrutin public, là encore disponible sur le site de l'Assemblée nationale, indique les noms des votants et ce pour quoi ils ont voté, tout en les affiliant à leur groupe politique et en recensant, dans un «camembert », l'équilibre du vote global. La présentation plus basique sur le site du Sénat en rend la lecture et l'analyse moins facile.

De même, dans un souci de contrôle, des éléments sont parfois diffusés, sans que le public en ait véritablement connaissance ou qu'il soit en mesure de les lire facilement. On peut citer deux exemples. D'une part, alors que l'affaire Fillon défrayait la chronique pendant la campagne présidentielle de 2017, peu savaient et ont rappelé que le Sénat diffusait, depuis de nombreuses années déjà et avant même l'obligation de transmission à la HATVP, le nom de tous les collaborateurs des sénateurs. D'autre part, alors que l'on a récemment abaissé la réserve parlementaire au rang de prébende scandaleuse ${ }^{23}$, on oublie qu'elle avait recouvré de véritables garanties de transparence depuis qu'elle est publiée sur les sites des Assemblées. Toutefois, la lecture de l'information est peu aisée: si le nom du parlementaire et du bénéficiaire sont mentionnés, aucune autre information n'est apportée (en dehors du montant). Si bien qu'il faut ensuite mener une recherche sur le bénéficiaire pour tenter de déterminer ses liens éventuels avec le parlementaire et la nature du projet soutenu. Il n'en demeure pas moins que, si la volonté d'ouverture ne se traduisait pas de façon aussi évidente, elle pouvait être facilement améliorée sans nécessité de supprimer cette réserve bien utile aux collectivités territoriales et au monde associatif.

\footnotetext{
20 Sur la transparence au Parlement, cf. G. Bergougnous, «Le Parlement et la transparence », in B. MAthieu, M. VerpeAux (dir.), Transparence et vie publique. Neuvième Printemps de droit constitutionnel, Dalloz, Paris, coll. Thèmes et commentaires. Cahiers constitutionnels de Paris I, 2015, p. 53-70.

21 Le compte rendu des séances du Parlement qui s'est réuni en comité secret au cours de cette période est ainsi accessible, désormais. 22 Articles $63 \mathrm{du}$ Règlement de l'Assemblée nationale (RAN) et 61 du Règlement du Sénat (RS).

23 L'article 14 de la Loi organique $\mathrm{n}^{\circ}$ 2017-1338 du 15 septembre 2017 pour la confiance dans la vie politique (JORF 16 septembre 2017 ) la supprime.
} 
Parallèlement, soucieuse de s'ouvrir au maximum aux citoyens afin de leur offrir tous les moyens de mener un contrôle direct ou d'obtenir une information de façon immédiate, l'administration se livre à ce que l'on appelle l'Open Data ou l'accès ouvert aux données, qui permet de diffuser librement des données. En France, la loi pour une République numérique a créé un «service public des données de référence ${ }^{24}$, désormais prévu par le code des relations entre le public et l'administration ${ }^{25}$. L'objectif assigné par la loi à ce service public est «de faciliter [la] réutilisation» de ces données de référence, que ce soit par les administrations ellesmêmes que par les citoyens. Cela est complété par un site institutionnel, dont la création a été décidée en $2010^{26}$ et qui répertorie la documentation publique officielle, de l'État au sens le plus large puisque cela inclut les collectivités territoriales, les établissements publics, les services publics. Cet outil va encore plus loin dans la démarche puisqu'il permet à chaque «citoyen », après inscription, «de produire, d'enrichir ou de compléter ces données ou de partager des données d'intérêt public, qu'il s'agisse par exemple de l'inventaire d'une bibliothèque communale, de l'état de la voirie, de la composition nutritionnelle de produits alimentaires ou du bilan environnemental d'une entreprise $»^{27}$.

L'objectif initial est louable: faire bénéficier des données publiques au plus grand nombre et même idéalement à tous. D'autant plus louable que l'ouverture est dédoublée: dans l'obtention de l'information et dans la fourniture de l'information. Toujours aussi louable grâce à la prise en compte de la législation sur la protection des données personnelles ${ }^{28}$, renforcée par le règlement général de la protection des données (RGPD), de l'Union européenne ${ }^{29}$, applicable à compter du 25 mai 2018. Toutefois, le résultat est-il aussi palpable que l'objectif est louable? La jeunesse du dispositif interdit d'apporter une réponse tranchée, mais impose une appréciation nuancée. D'abord, le nombre de sources d'information risque de tuer l'accès à l'information. Ce n'est pas tant l'abondance des informations qui soulèvent des difficultés, car l'on n'est jamais trop informé. En revanche, la démultiplication des plateformes numériques rend la tâche ardue à l'utilisateur qui ne sait plus où ni quoi vraiment chercher : sites des institutions (Gouvernement, Présidence de la République, Assemblées, juridictions, etc.), Légifrance, Service-public.fr, Data.gouv.fr, sites des AAI, etc. Cela nuit à la qualité de l'accès à

24 Article 14 de la loi no 2016-1321 du 7 octobre 2016 pour une République numérique, fORF du 8 octobre 2016.

25 Art. L. 321-4 et R. 321-5 et s. CRPA.

26 Il s'agit de www.data.gouv.fr. Voir la Circulaire du 26 mai 2011 relative à la création du portail unique des informations publiques de l'État «data.gouv.fr» par la mission «Etalab» et l'application des dispositions régissant le droit de réutilisation des informations publiques, JORF 27 mai 2011. Etalab est une mission créée par l'État et chargée de concevoir ce portail unique interministériel «data.gouv.fr » et de coordonner l'action des administrations de l'État en matière de réutilisation des informations publiques. Créée par le décret n $2011-194$ du 21 février 2011, elle est placée sous l'autorité du Premier ministre et rattachée au secrétaire général du Gouvernement.

27 «Découvrir l'Open Data en tant que citoyen», https://doc.data.gouv.fr/faq/citoyen/ (consulté le 12 avril 2018).

28 Définition en est donnée, en France par l'article 2 de la loi n ${ }^{\circ}$ 78-17 du 6 janvier 1978 relative à l'informatique, aux fichiers et aux libertés, fORF 7 janvier 1978, p. 227. Il s'agit de «toute information relative à une personne physique identifiée ou qui peut être identifiée, directement ou indirectement, par référence à un numéro d'identification ou à un ou plusieurs éléments qui lui sont propres ». La Cour de cassation a récemment précisé «que les adresses IP, qui permettent d'identifier indirectement une personne physique, sont des données à caractère personnel», Civ. $1^{\text {ère }}, 3$ novembre 2016, $\mathrm{n}^{\circ} 15-22.595$.

29 Règlement (UE) 2016/679 du Parlement européen et du Conseil du 27 avril 2016 relatif à la protection des personnes physiques à l'égard du traitement des données à caractère personnel et à la libre circulation de ces données, et abrogeant la directive 95/46/CE (règlement général sur la protection des données), FOUE 4 mai 2016, L 119/1; cf. D. DA PALMA, « La mise en conformité du traitement des données personnelles dans l'Open Data au regard du Règlement général sur la protection des données. Quels enjeux pour les collectivités territoriales? », in $\mathcal{F} C P$ - $A$ 2017, 2217. 
l'information. De surcroît, la possibilité offerte à chaque « citoyen» de publier de l'information permet à n'importe qui (inutile d'être citoyen au sens juridique, mais simplement un utilisateur d'internet, disposant d'une connexion et d'une adresse mail... et un même individu peut en créer autant qu'il souhaite) de publier n'importe quoi (à l'exception de données protégées par la loi, du fait d'une modération sur le site). Cela nuit, cette fois, à la qualité de l'information elle-même. Cette diversité d'informations et de leurs accès nuit, in fine, au contrôle, car c'est un tort de croire que d'informations démultipliées naît un contrôle renforcé.

Pourtant, cette diffusion élargie et renforcée est, là encore, institutionnellement organisée : ce sont les institutions qui diffusent et autorisent la diffusion de données publiques. Il serait alors temps de transformer la diffusion contrôlée en diffusion rationalisée (mais non limitée), afin d'assurer un passage concomitant de cette diffusion contrôlée à un contrôle de et sur la diffusion. La rationalisation de la diffusion se réaliserait alors non dans sa limitation mais dans sa systématisation, offrant à l'utilisateur davantage de pédagogie dans l'accès aux données. Pour cela, il faut cesser de diffuser et créer des sites en se plaçant du point de vue du diffuseur, pour se placer du point de vue de l'utilisateur... et du citoyen.

Ce dernier, entendu comme citoyen ou simple individu, peut alors se saisir des informations diffusées afin de faire subir aux gouvernants un contrôle assuré.

\section{Subir assurément le contrôle}

Le numérique devient un outil d'enquête facilitant le contrôle (A), mais il requiert d'être relayé pour produire des effets efficaces (B).

\section{A. Un outil d'enquête}

Le numérique est un outil d'enquête, directement utilisé par le citoyen (1) ou indirectement organisé par des collectifs (2).

1. Grâce à l'accès à l'information diffusée par les gouvernants, les gouvernés peuvent mieux contrôler les premiers, en menant des enquêtes à leur égard: cela donne lieu à des «veilles citoyennes». Elles débouchent sur de nouveaux mécanismes, comme la «webocratie de contrôle $»^{30}$ ou la «sousveillance», en lien avec la «surveillance $»^{31}$. Effectuée par les citoyens, elle illustre la verticalité ascendante de ce mécanisme de contrôle des gouvernants. Selon le rapport vertical classique entre l'électeur et l'élu, le représenté et son représentant, le gouverné et le gouvernant, le premier est placé « sous» le second. Ainsi, il (sous)veille et contrôle alors ce que font les gouvernants. Déjà possible avec des mécanismes classiques, cette sousveillance

30 N. Berggruen, N. Gardens, Gouverner au XXIe siècle, Fayard, Paris, 2013.

31 On doit notamment cette notion à Steve Mann, scientifique canadien mais non juriste, puisqu'il est professeur de sciences appliquées. Cf. S. Mann, J. Nolan, B. Wellmann, «Sousveillance: Inventing and Using Wearable Computing Devices for Data Collection in Surveillance Environments », in Surveillance \& Society, [S.1.], v. 1, n. 3, p. 331-355, sep. 2002. ISSN 1477-7487, disponible en ligne: https://ojs.library.queensu. ca/index.php/surveillance-and-society/article/view/3344 (consulté le 12 avril 2018). 
est renforcée grâce à l'outil numérique, amorçant ce contrôle démocratique sans fin, sans limite et sans frontière, initialement esquissé.

Le numérique offre d'abord un dépassement des frontières classiques, élargissant les catégories de gouvernants sousveillés et de gouvernés sousveillant, ce qui renforce directement le contrôle démocratique. En effet, la relation traditionnelle entre le citoyen et «son » représentant, celui qu'il a élu, est dépassée. L'utilisation d'un contrôle numérique, par l'accès et l'exploitation de l'information, ne requiert pas de relever de la catégorie juridique de citoyen : elle est ouverte à toute personne disposant d'un accès à internet. Nul besoin, donc, d'être électeur, d'être national, de résider sur le territoire de la circonscription concernée. De surcroît, ce contrôle peut être exercé à l'égard de tout gouvernant et non seulement à l'égard de «son » représentant, c'està-dire celui issu de la circonscription dans laquelle on vote (si l'on est électeur).

Outre le dépassement des frontières, l'outil numérique favorise un éclatement des limites d'autrefois. Auparavant, il n'était pas toujours aisé de suivre l'action et les activités des gouvernants : la diffusion numérisée de ces dernières en facilite désormais le suivi, on l'a souligné. Surtout, le contact devient plus direct, car dématérialisé, sans qu'il soit nécessairement personnalisé car l'on ne sait pas qui se cache derrière le profil numérique, entre le gouvernant luimême et son équipe. Mais là où il fallait soit écrire une lettre, soit décrocher son téléphone, on peut aujourd'hui facilement envoyer un courrier électronique ou apostropher sur un réseau social. Les gouvernants sont ainsi interrogés ou interpellés, directement et publiquement, par l'intermédiaire de ces réseaux sociaux. Ils peuvent l'être de façon organisée, s'ils décident de se confronter à un échange avec les utilisateurs. Ils peuvent l'être, aussi, de façon spontanée, lorsqu'un utilisateur poste un commentaire ou réagit. La publicité attachée à ces interpellations peut leur donner un impact important, mais les dilue également dans la masse, tendant à la faire disparaître et à en dissiper les effets. Il est alors indispensable, pour qu'il y ait des conséquences utiles, que lesdites interpellations soient vues, lues et relayées. Tout cela permet une accélération des échanges ou une démultiplication des correspondances (le même courrier peut être adressé à une multitude de destinataires en quelques clics seulement, quoique ce ne soit pas là l'arme la plus efficace du contrôle, en raison de son caractère confidentiel et bilatéral).

L'outil numérique contribue à développer un contrôle sans fin, car l'enquête peut être (in) utilement réutilisée. Il sert d'abord un contrôle individuel, exercé par chaque individu qui dispose d'un accès à internet et peut consulter les sites, notamment institutionnels.

2. Cette sousveillance peut ensuite subir une forme d'institutionnalisation ou d'organisation, la faisant passer d'un contrôle individuel direct à un contrôle collectif indirect (puisqu'il passe par l'intermédiaire de la plateforme d'institutionnalisation). Des plateformes électroniques voient alors le jour, alimentées par des collectifs d'individus, des associations, voire des lobbies. Elles agrègent les informations disponibles à partir des sites institutionnels ou personnels 
des gouvernants, afin de les rendre plus lisibles et plus pédagogiques, au risque de devenir, à leur tour, démagogiques.

Tel fut le cas de WikiLeaks, sans doute le plus célèbre. C'est ce que fait, en France, le site regardscitoyens.org, qui a débouché sur nosdeputes.fr et nossenateurs.fr. Ces sites permettent, grâce à des graphiques et autres données systématisées et lisibles, de détailler et retracer l'action (ou l'inaction) des parlementaires. On peut ainsi facilement savoir quels sont les centres d'intérêt de tel ou tel parlementaire, son degré de présence au Parlement, le nombre de questions posées, de propositions déposées, d'interventions exposées, etc. De même, il est possible de suivre un projet ou une proposition de loi ou une séance, afin de savoir comment il a été discuté, pendant combien de temps, quels termes ont été le plus ou le moins répétés.

Tout cela conduit à des données principalement statistiques que le gouverné peut utiliser pour exercer un contrôle assidu et assuré des gouvernants. La sanction de ce contrôle peut être immédiate, par des mises en cause qui ne peuvent prospérer que si elles connaissent un relais, principalement médiatique. Cela soulève alors la question de la place des médias dans ce processus de contrôle, tant au niveau de leur rôle de relais et de contrôleur, qu'à celui de leur connexion avec les gouvernés qui lancent une alerte. La sanction peut également être différée, lors des échéances électorales suivantes: l'électeur ainsi informé sera sans doute davantage éclairé dans son choix et, par ce dernier, pourra sanctionner l'élu qui est à nouveau candidat ou, à défaut, son action endossée par son parti politique.

Néanmoins, cette course à la transparence et à la statistique cache une double réalité car, sous prétexte de rendre plus lisible, elle peut rendre illisible et occulte l'invisible. D'une part, le chiffre n'est pas synonyme d'efficacité, au contraire: la statistique peut donc être trompeuse. La multiplication des actions tend à les diluer, tandis que des actions ciblées et choisies peuvent davantage prospérer. Ainsi, un parlementaire qui posera moins de questions ou déposera moins de propositions de loi ou d'amendements ne sera pas pour autant moins efficace que celui qui fera statistiquement «du chiffre», car il pourra déployer ses efforts pour les faire prospérer. Or la simple statistique, brute, n'en rend pas compte: sous prétexte de rendre lisible, elle ne détaille pas les actions et les rend, en définitive, illisibles. L'outil, malgré sa vocation pédagogique, peut alors rapidement se révéler démagogique, d'autant plus si les gouvernants eux-mêmes se posent en relais de cette démagogie. En effet, se sachant «sousveillés», ils peuvent simplement se livrer à un accroissement brut de leurs actions et démarches (facilité par la magie du copier/coller...), inutilement diluées, au détriment d'actions utilement ciblées.

D'autre part, ces études ne peuvent s'appuyer que sur ce qui est répertorié, donc officiel et institutionnel. Or le travail des parlementaires, par exemple, ne se déroule pas exclusivement dans l'hémicycle, dans une commission ou dans le dépôt d'une proposition de loi, d'un amendement ou d'une question. Il y a d'abord les réunions de groupe, essentiel à la coordination, où nul d'extérieur ne peut effectivement savoir qui siège et ce qui se déroule à l'intérieur (sauf 
à être informé par des voies non officielles ou des communiqués, mais ce n'est plus de l'information institutionnalisée). Ensuite, il y a le travail en circonscription, que ce soit le contact public avec les électeurs que les rendez-vous, réunions, ou autres interventions permettant au représentant d'être à l'écoute de ses représentés et de faire droit à leur demande. Enfin, il arrive aussi que les gouvernants travaillent tout bêtement... dans leur bureau. S'il en est certains qui font écrire leurs rapports, propositions de loi, amendements et discours par leurs collaborateurs ou par des administrateurs, il en est d'autres qui déploient un véritable travail de fond, relisant, corrigeant et enrichissant substantiellement le travail qui leur est soumis. Là encore, au prétexte de rendre lisible, l'étude statistique à partir de données transparentes occulte l'invisible, pourtant essentiel.

\section{B. Un contrôle relayé}

L'outil numérique, les plateformes qu'il favorise et les mécanismes auxquels il donne lieu permettent de renouveler les enquêtes menées à l'égard de l'action des gouvernants et d'actualiser (presque au sens propre de la page internet) le contrôle issu des articles 14 et 15 de la Déclaration de 1789 en une forme de « contrôle 2.0 ». Cependant, cela ne constitue qu'un relais : en guise de contrôle renouvelé, on se confronte à un contrôle doublement relayé, par les gouvernés (1), puis par les gouvernants (2).

1. En effet, ce contrôle actualisé correspond à la reprise de mécanismes traditionnels (donc anciens), par des moyens et des acteurs modernes. Classiquement, le contrôle des gouvernants relève des gouvernants. C'est à la fois l'esprit de la démocratie représentative, la raison d'être des parlements et la logique de la séparation des pouvoirs, avec le glissement vers une articulation entre la majorité et l'opposition. Tout cela n'est remis en cause ni par la modernité ni par le numérique. Le Gouvernement demeure responsable devant le Parlement (article 20 de la Constitution), le Parlement continue de contrôler l'action du Gouvernement (article 24), l'administration doit toujours rendre compte de son action (article 15 DDHC). Les mécanismes classiques (questions orales, écrites, d'actualité, comité d'évaluation, missions d'information, commissions d'enquête, contrôle sur pièce et sur place, pour ne citer que ceux-ci) sont plus que jamais d'actualité, à l'heure où l'on prône la démocratie exemplaire.

Malgré tout, dans cette tâche, les gouvernants peuvent être relayés par les citoyens: c'est le premier relais. Ces derniers peuvent alerter, diffuser, se coordonner pour mener des enquêtes. Si le numérique n'est pas un outil indispensable, il est un vecteur d'accélération incontestable. Il facilite l'accès aux sources, favorise la coordination des actions et renforce la diffusion des enquêtes. À ce sujet, le phénomène dit du fact checking est un exemple topique. Initialement, il s'agissait de blogueurs qui se saisissaient de faits avancés par des responsables politiques, pour les vérifier et, généralement, les affiner ou les contredire. Puis, cela a été accaparé par la 
presse, des journaux créant des rubriques en leur sein avec vocation de «décoder $»^{32}$ et vérifier les dires des gouvernants. Ce fact checking peut dévier en fast checking, avec des vérifications en temps réel qui, elles, ne sont rendues possibles que par le numérique. La rapidité et la disponibilité de la connexion internet, la recherche efficace d'une information ciblée, pourvu qu'elle soit fiable, permet de vérifier sans délai le propos d'un responsable politique et, le cas échéant, de réagir. Le contrôle des gouvernants, qui était autrefois exclusivement entre les mains des gouvernants qui se contrôlaient donc eux-mêmes, descend entre les mains des gouvernés, qui viennent ainsi l'exercer non en leur lieu et place mais en complémentarité.

2. Ce premier relais renouvelle les enquêtes, dans le cadre d'un contrôle démocratique: il resserre le lien entre gouvernants et gouvernés, il a vocation à renforcer l'exemplarité des premiers et il élargit les sources de contrôle. Il demeure toutefois de faible portée s'il n'est pas lui-même relayé: c'est la nécessité d'un second relais. Car une fois que les individus, qu'ils soient citoyens ou non, organisés ou non, ont exercé et diffusé leur enquête, encore faut-il qu'ils touchent une cible. La cible ultime est le(s) gouvernant(s) qu'ils contrôlent. Mais pour que cette cible soit touchée, leur enquête et leur contrôle doivent être relayés: par d'autres individus, par des médias ou par d'autres gouvernants. Et ce ne sont que ces derniers qui peuvent constituer un second relais efficace quant aux effets du contrôle, car ils ne peuvent pas être activés par les gouvernés eux-mêmes : c'est toute la logique de la complémentarité du contrôle, lequel «a cessé d'être unilatéral pour devenir bidimensionnel, si pas circulaire $»^{33}$. Le fonctionnement de la démocratie demeure essentiellement représentative, donc fondamentalement institutionnelle.

Ainsi, entre la sanction immédiate (relais et pression médiatiques) et la sanction différée (lors d'échéances électorales), se trouve la sanction relayée par d'autres gouvernants. Dans notre système, il n'existe pas de mécanismes permettant une prise directe du gouverné, usager du numérique, sur le gouvernant. Mais le premier peut activer un processus qui devra être relayé par les autres gouvernants. En définitive, le numérique constitue un outil de contrôle efficace des gouvernants, mais ce n'est que par le relais institutionnel qu'il en devient un outil effectif. Cela n'est alors pas sans soulever la question de son efficacité réelle : il n'est pas tant un outil de contrôle des gouvernants, que ce soit sur les gouvernants ou par les gouvernants, qu'un outil au service du contrôle des gouvernants, que ce soit au service du contrôle sur les gouvernants, ou par les gouvernants.

\footnotetext{
32 On peut citer, parmi d'autres exemples, la rubrique «Les Décodeurs» du journal Le Monde, qui était initialement un blog (en 2009). On peut aussi citer «Les Pinocchio de l'Obs». Cela a également irrigué les chaînes de radio, «Le vrai du faux» de France Info ou «Les faux de l'info » d'Europe 1. Mais les journalistes n'ont pas l'exclusivité: on salue ainsi l'initiative d'un collègue, Vincent Couronne, qui a lancé le blog Les Surligneurs en janvier 2017, en lien avec le centre de recherche en droit public Versailles Institutions Publiques.

33 A. Piraux, «Les outils numériques et la réinvention du fonctionnement de l'État», in Pyramides. Revue du Centre d'études et de recherches en administration publique, 2016, 26/27, § 11 .
} 



\section{DISCUSSION $^{1}$}

Public (François-Bernard Huyghe): Une question pour Madame Vidal-Naquet. Il me semble que les actions liées aux contestations ou aux pétitions portent deux problèmes. Le premier problème c'est celui du «faux». Nous avons tous entendu parler de ces pétitions signées par Shakespeare, Jaurès, etc. C'est un phénomène accentué par l'apparition de la technique d'astroturfing, l'utilisation d'algorithmes qui simulent l'activité d'êtres humains. Deuxième point, le contenu même de ces pétitions ou de ces demandes qui peuvent être uniquement fantasmatiques ou imaginaires. Je pense à l'expérience au Danemark où les universitaires ont obtenu énormément de signatures à une pétition pour ne pas déplacer une statue historique alors que c'était un canular. Non seulement les participants peuvent ne pas exister, mais leur degré de responsabilisation peut varier, ce que Evgeny Morozov appelle le «slacktivisme», l'activisme paresseux: on s'indigne d'un clic pour un sujet qui n'entraîne guère de conséquence dans notre vie, dans la réalité. Ce sont deux questions auxquelles très honnêtement je n'ai aucune réponse.

Ariane Vidal-Naquet: Je n'ai pas franchement de réponse non plus. Sur la vérification des participants au processus, concrètement, je ne sais pas comment il faudrait procéder. Alors que l'on parle du principe de citoyen-législateur fondé sur l'article 6 de la Déclaration des droits de l'homme et du citoyen, n'importe qui peut être législateur, n'importe quel être fantasmagorique ou n'importe quel citoyen du monde entier. Il est possible d'avoir une vision très universaliste en se réjouissant que les internautes coréens puissent participer à la loi sur le numérique. Par rapport à la loi Lemaire, j'ai été surprise que cette loi ait été ouverte au monde entier et qu'il y ait eu beaucoup de contributions étrangères sur internet.

En ce qui concerne le contenu des pétitions, il y en a de très fantaisistes notamment sur le site de la Maison-Blanche («il faut déplacer la fête d'Halloween un samedi» par exemple) ou de très sérieuses (« déclarer le Ku Klux Klan comme organisation terroriste »). Le problème est que les pétitions délirantes nécessitent aussi une réponse de la Maison-Blanche car elles ont atteint les 100000 signatures. Les services de la présidence devront donc expliquer pourquoi cela n'est pas possible.

S'agissant de l'initiative citoyenne européenne, c'est très sérieux; les propositions sont de vraies propositions sérieuses: le nombre de signatures nécessaires et la répartition géographique ont un impact certain. A contrario, pour les États-Unis, 100000 signatures ce n'est rien.

Public (Pascal Jan): Les exposés ont été très riches, très intéressants, mais ils soulèvent pour moi de nombreuses questions. Première chose, de quel citoyen parle-t-on? Le citoyen, cela veut dire quelque chose juridiquement. On parle d'e-démocratie, de démocratie connectée, on se réfère toujours au citoyen, mais sans véritablement définir qui est le citoyen. Dommage d'ailleurs qu'Axelle Lemaire ne soit pas là car nous aurions pu avoir un échange très vif: avec la loi sur le numérique,

1 Les coordonnateurs souhaitent remercier Robin Trabut, doctorant contractuel et membre du CDPC Jean-Claude Escarras de la Faculté de droit de l’Université de Toulon, qui a assuré la retranscription des débats ici publiés. 
beaucoup de connexions et d'interactions correspondaient à du lobby. C'est le problème de l'identification IP et autres, desdits citoyens, qui ne sont en rien des citoyens. C'est tout simplement la traduction par un autre moyen de la possibilité d'influencer la rédaction de la loi ou d'un décret. Le décret de la loi sur la transition énergétique a été ouvert à la consultation. Le problème ne concerne pas que le législateur, mais aussi le pouvoir réglementaire. Il y a eu un vrai problème, parce que ce sont des «citoyens» qui avancent cachés, masqués, pour modifier la loi. Et cela pose le problème du lobbying. Je ne vois absolument aucun inconvénient à ce que ce soit d'une transparence la plus totale, je pense que cela fait partie de la confection de la loi, mais faut-il encore l'assumer. Parce que là on se réjouit d'une participation, mais laquelle? Cela me pose déjà un problème: qui intervient à l'encontre des gouvernements? Après, je suis tout à fait d'accord avec toi Jean-Philippe sur la façon dont tu as présenté les choses.

Le second point, j'ai l'impression que la question de la démocratie connectée ou numérique nous fait retrouver un vieux débat: cette démocratie serait confisquée par les élus, c'est donc une critique très sérieuse, cinglante par rapport à ce que sont les élus, ce qu'ils représentent, ce qu'ils peuvent faire. Cela est souvent dit, mais je trouve qu'on ne va pas assez loin dans cette approche-là. J'ai l'impression, à lire puis à vous écouter tous les deux, que l'on est face à une sorte de «village de citoyens », quelque chose à côté des structures traditionnelles. Finalement, est-ce que quelque part ce n'est pas la revanche de Rousseau contre Sieyès? Est-ce que quelque part - Rousseau c'est la démocratie, Sieyès c'est le régime représentatif - en incluant le citoyen à chaque instant, ce dernier peut agir sur la norme et éventuellement créer une fièvre normative? À chaque instant, le citoyen peut agir sur la norme comme les assemblées primaires, pour faire court, dans le projet de 1793.

Dernier élément sur le contrôle, lorsque l'on relit les écrits et que l'on analyse bien la pensée de Sieyès, le régime représentatif est lié chez lui à l'émergence de la société commerciale. Aujourd'hui, il est souhaité que les citoyens participent tout le temps - fièvre normative -, mais est-ce qu'il en a le temps? Comment peut-il trier l'information, comment peut-il hiérarchiser l'information, comment peut-il s'assurer de la pertinence de l'information? Cela me pose un vrai problème. Il s'agit d'une démocratie réactive, même si, certes avec la loi Lemaire c'est une démocratie «participative », ou au moins la participation directe à une œuvre normative. En tout cas, c'est plus pour critiquer que pour approuver, c'est donc à sens unique. Nous n'insistons pas assez par rapport aux réseaux sociaux, nous sommes dans un total enfermement: nous sommes des amis des amis, nous reproduisons les critiques des critiques, nous nous enfermons donc dans une information, nous ne sommes pas du tout objectifs pour apporter une vision critique par rapport à la norme. Cela traduit un certain malaise par rapport à cela, non pas que ce ne soit pas nécessaire, mais cela peut déstructurer, en tout cas cela affaiblit considérablement les institutions représentatives. Je suis assez critique, en tout cas, je ne suis pas optimiste par rapport à cette démocratie.

Public: Je m'intéresse au numérique avec des Japonais, des francophones, des Canadiens, des Américains, des Sud-américains, nous avons une vision assez globale d'internet et le mot qui revient le plus souvent est «ambivalence». Bien sûr que les citoyens vont de plus en plus participer, 
moi aussi en tant que citoyen j'ai cliqué sur des sites, mais je ne clique plus, parce que je n'ai plus le temps de cliquer! Parce que cela prend du temps de cliquer, et cela prend du temps sur ma réflexibilité, sur ma réflexion personnelle, sur mon for intérieur même. La question du temps est essentielle: on parle du speed dating, de «ma thèse en 180 secondes », mais jusqu'où va-t-on aller? Nous allons arriver à ce que la foule remplace le peuple comme l'écrivait Victor Hugo. Cela aboutit, comme aux États-Unis, à l'arrivée de Donald Trump au pouvoir. Il faut regarder les choses en face, internet c'est ambivalent, c'est un monde de surveillance: 300000 Chinois sont payés par le parti communiste pour surveiller le monde d'internet. C'est un monde de réseau fermé parce qu'on est dans l'entre-soi. Le monde de l'internet est un miroir déformé. Tout le monde n'est pas sur le net en permanence, et il y a même un commencement de recul, il y a des jeunes qui ne sont plus aussi connectés qu'avant car ils ont été en «surchauffe informationnelle». Ma question rejoint le malaise de notre hypermodernité : jusqu'où va-t-on aller dans la rétractation du temps? Pour réfléchir, il faut du temps... quel bonheur d'avoir quelques jours pour soi.

Public: Juste des réflexions générales, je partage ce qui a été dit auparavant. Il me semble que la question qui se pose aujourd'hui est de savoir si de nouvelles modalités techniques permettent de mieux réaliser une ambition d'organisation sociale: la possibilité pour les citoyens de produire les normes qui leur sont destinées. Ces techniques permettent-elles de pallier les crises de la démocratie actuelle, notamment la crise de la représentativité? Ce qu'a dit Ariane Vidal-Naquet clôt finalement le débat. Il y a un paradoxe du numérique, de ce point de vue, puisqu'il est censé améliorer la représentativité alors même que les participants, par internet, ne sont pas représentatifs. En affirmant qu'internet permet une meilleure démocratie, on compense le manque de représentativité... mais il n'y en a aucune. Je trouve inquiétant que l'on s'en remette à internet, à la démocratie numérique, alors que la représentativité en est absente. Est-ce que ce n'est pas un moyen de ne pas régler les problèmes? Au contraire, je pense que cela renforce les problèmes: quelle représentativité? Qui vote? C'est une «arnaque» totale en réalité que de laisser croire que cela peut remplacer la logique représentative, c'est illusoire et cela cache les enjeux de pouvoir, ce qui est très inquiétant.

Ariane Vidal-Naquet : Je vais faire une réponse collective sur la crise de la représentativité et le manque de représentativité des participants. Sur le lobbying, c'est intéressant puisque la plupart des organismes qui promeuvent la co-législation estiment qu'il n'y aura pas de lobbying. C'est aussi ce que défend Axelle Lemaire. Justement, dans le rapport d'application (qui s'apparente plus à une enquête) sur la loi Lemaire, il y a eu toute une série de questions posées à ceux qui ont participé: qui êtes-vous? Homme ou femme? Quel âge? Représentation institutionnelle ou non? Derrière cette enquête, il y a bien la conscience qu'il y a des associations et donc du lobbying. Je me dis que cela dépend quand même du sujet abordé par rapport au «taux de lobbying ». Pour le droit de pétition, il semble que cette immixtion du lobbying est assez nette. Pour les études d'impact qui accompagnent les projets de loi, on a l'impression qu'il y a beaucoup de contributions qui se recoupent et qui sont rédigées de la même manière, mettant en évidence qu'il y a eu derrière une influence bien plus institutionnelle. Par contre, pour les contributions à la loi Leonetti sur la fin de vie, c'est la «Cour des 
Miracles» («mon chien est mort, c'est horrible »...) : il y a très peu de contenu juridique, et curieusement, on ne voit pas apparaître de lobbying, en tout cas ce n'est pas visible.

Pour la revanche de Rousseau sur Sieyès: oui et non. En réalité, dans tous ces mécanismes, le pouvoir reste entre les mains du législateur. En réalité, où que ce soit dans le monde, ces initiatives n'aboutissent pas. Je m'avance beaucoup, évidemment je n'ai pas fait une étude exhaustive, mais dans ce que j'ai étudié il n'y a qu'une initiative qui a abouti, c'est peu. C'est un «gadget institutionnel »: on croit que cela va révolutionner la chose, il n'en est rien; mais c'est très embêtant de dire que les résultats sont très mitigés ou très faibles, alors que l'on est en train d'instiller le ver dans le fruit, je vous rejoins donc complètement.

Président (Jean Gicquel): Je saisis l'opportunité offerte par ce rapprochement entre Rousseau et Sieyès, pour dire que cela me fait songer à la fameuse intervention dans le domaine des idées politiques de Saint-Simon, l'État industriel, avec l'apologue des abeilles et des frelons; dans le sens de dire que les représentants au sens ordinaire du terme sont des parlementaires avec un rôle très mineur. C'était peut-être hardi comme rapprochement Saint Simon et le numérique. L'un des grands thèmes aujourd'hui c'est la trahison des élites. Aujourd'hui, on entend, on lit que les élites ne s'intéressent plus ni au pouvoir politique, ni à l'ENA, mais à l'argent. Est-ce que c'est vrai, est-ce que c'est faux? Est-ce qu'il y a une relation de cause à effet? Peut-être que l'on ne va pas refaire le monde, même si c'est possible en colloque. Cette trahison des élites apparaît de plus en plus présente dans le débat des idées.

Pascal faisait allusion au fait que l'on s'intéresse beaucoup au domaine législatif, mais qu'on doit s'intéresser aussi au domaine réglementaire. Vous savez que chaque année, c'est une spécificité sénatoriale, a lieu un débat sur le bilan du décret d'application. Dernièrement, il y avait notamment un rapport où le Secrétaire général du Gouvernement expliquait comment le délai raisonnable est respecté entre le moment où la loi est promulguée et la publication des décrets. Il indiquait notamment que la première étape, la plus importante, au lendemain du vote, c'est la rencontre avec les représentants des forces vives de la Nation. En bref, il s'agit de la réunion systématique, à Matignon, des groupes de pression intéressés... et cela va tout à fait dans le sens que Pascal a indiqué.

Jean-Philippe Derosier: Je n'ai pas grand-chose à ajouter sur ce qui a été dit. Sinon tempérer ce qui a été dit sur Donald Trump et qu'aucun réseau social ne l'avait prédit. Certains l'avaient prédit, mais personne n'a voulu les écouter. Dès l'été 2016, Michael Moore avait très précisément démontré que Donald Trump allait gagner (démonstration démocratique très intéressante et très fouillée) et personne ne l'a écouté. Enfin, peut-être que si, mais moi-même j'étais prêt à mettre mon billet sur le fait qu'Hillary Clinton allait gagner. Il y a aussi des chercheurs qui avaient démontré que Donald Trump allait gagner, mais personne ne les a écoutés, donc c'est à prendre au sérieux.

En ce qui concerne les lobbies - un sujet qui me passionne -, en France, c'est un peu un «gros mot», donc personne ne veut le prononcer, ni les lobbyistes eux-mêmes ni les institutionnels gouvernants, donc puisque personne ne veut le prononcer, personne ne veut s'en saisir. Puisque personne 
ne s'en saisit, cela devient forcément un «gros mot» parce que c'est la pagaille, les lobbyistes deviennent de «grands méchants». Quand on dit lobbies, on pense tabac, et quand on pense tabac, on pense santé publique. Donc les lobbies veulent tous nous tuer, pour aller très vite. Mais les lobbies c'est bien, c'est même indispensable, je suis un grand défenseur des lobbies. Heureusement qu'il y a des lobbies pour structurer un petit peu le fonctionnement de la démocratie. J'ai toujours soutenu que les lobbies sont à la démocratie ce que les groupes parlementaires sont au Parlement. C'est-à-dire, qu'ils structurent par courant d'idées, par groupe d'intérêt (intérêt politique au sein des groupes parlementaires au Parlement et des intérêts économiques au sein des lobbies); même s'il ne faut pas leur livrer les clés de la démocratie et que cette dernière ne soit plus qu'une démocratie des lobbies. Mais à l'inverse, que les acteurs économiques n'aient absolument aucune partie prenante dans le monde démocratique serait également terrible. À partir du moment où on légifère, ce qui vient d'être fait, sur les lobbies, que leur existence est reconnue, qu'ils sont encadrés, pour éviter que ce soit la pagaille; et en outre, il n'y a pas que des lobbies du tabac ou des opérateurs économiques. Rien n'empêche de faire un groupement d'intérêt sur les intérêts démocratiques, les intérêts de la société civile. Même si je n'aime pas trop ce terme, cela distingue société militaire et civile, et par conséquent les politiques sont de la société civile. Quand il existe des plateformes comme celles que j'ai mentionnées tout à l'heure, cela peut être apparenté à une forme de lobby. Le collectif « Nous citoyen » qui voulait introduire un candidat à l'élection présidentielle qui serait issu de la société civile là encore, cela peut être une forme qui peut déboucher sur des lobbies, tout comme « Nuit debout».

Il s'agit d'autant d'éléments qui permettent de pallier ce problème de temps, car ce temps est effectivement un vrai problème. Nous sommes tous pris par ce problème de temps. Nous recevons une multitude d'informations que nous ne lisons pas, parce que nous n'avons pas le temps. Nous tous sommes les mieux placés pour effectuer un certain contrôle sur ce que font les gouvernants sans même être des responsables politiques justement, mais nous n'avons pas le temps. Au-delà de nous, professeurs de droit constitutionnel, les citoyens qui ne le sont pas, les citoyens normaux même si on l'est aussi, ont-ils la compétence pour le faire? Faire la loi, c'est un travail, et comme tout travail cela suppose des compétences, cela suppose au moins un savoir-faire. Contrôler les gouvernants cela suppose également des compétences. Il y a donc la question du temps, mais aussi de la compétence. Et là justement, les lobbies, à condition qu'ils soient encadrés et que l'on ne les considère pas comme des «gros mots », peuvent structurer tout cela parce qu'ils peuvent offrir un certain nombre de compétences et prendre en charge le temps (puisque cela deviendrait leur mission et cela serait dans leur intérêt) puisque le citoyen classique n'a pas le temps.

Les participations citoyennes à l'élaboration de la loi, c'est un «bal masqué », ce sont des lobbies évidents. Et même lorsque l'on parlait de la fin de vie, c'était du lobby: catholique ou médecin, mais pas forcément porté par eux. Les adresses IP ne servent à rien car ce sont des citoyens non «lobbiesés » qui sont en réalité saisis par les lobbies. Le lobby envoie le message à poster en demandant une légère adaptation. C'est pour cette raison que l'on a parfois 3000 fois le même message, et sur la fin de vie, c'était très flagrant. 
Pour terminer avec cette revanche de Rousseau et Sieyès : je pense qu'ils ne sont pas incompatibles. Là où la démocratie est exclusivement représentative, cela pose un certain nombre de problèmes, la démocratie exclusivement directe également. En revanche, un mélange des deux, associé à la démocratie connectée, me paraît faisable. Il est possible, là encore, non pas d'inventer, mais d'introduire des mécanismes numériques pour rafraîchir des mécanismes anciens; y compris des mécanismes de 1793 avec les assemblées citoyennes et la possibilité de validation référendaire à partir d'un certain nombre de signatures. Aujourd'hui, nous avons des moyens techniques suffisants pour permettre que tout cela passe par internet: nous payons nos impôts sur internet, nous pourrions faire des processus de validation ou d'invalidation de la voix par internet ou par des processus électroniques. Tout cela requiert évidemment un encadrement, cela ne se fait pas d'un claquement de doigts, mais tout cela me paraît possible et même indispensable.

Président (Jean Gicquel): Je voulais remercier notre collègue pour son intervention sur le temps et l'informatique. Et comme l'on m'a chargé des propos conclusifs et que je me demandais ce que j'allais dire, vous m'avez donné un espoir et je vous en remercie. Pour revenir sur ce qu'a dit Jean-Philippe sur le problème de l'élaboration de la loi, rappelons qu'il y a dans les assemblées des parlementaires dont chacun reconnaît la grande qualité. Mais nous avons découvert, au moment où les assemblées se sont ouvertes à la déontologie, que les collaborateurs, les assistants parlementaires, n'avaient pas toujours un rôle au service des représentants, mais représentaient des groupes de pression ce qui posait des problèmes de déontologie, au sens général du terme évidemment, mais aussi dans leur relation avec le député ou le sénateur. À ma connaissance, c'est une question qui n'a pas été tranchée dans le vif du sujet. 


\title{
GARANTIES DÉMOCRATIQUES ET NUMÉRIQUE
}

\author{
Sophie LAMOUROUX ${ }^{1}$
}

Pierre Rosanvallon écrit que la démocratie est à la fois «une promesse » et «un problème $»^{2}$. Une promesse car elle est toujours en devenir et ne satisfait jamais totalement ou même déçoit ses acteurs; un problème car il faut toujours inventer de nouveaux mécanismes afin que le sens de l'idéal démocratique ne s'épuise pas. De nombreux colloques et ouvrages abordent périodiquement la question de l'évolution de la nature et du concept de démocratie et des crises, déclins ou mutations dont elle ferait l'objet. C'est dans ce mouvement que doivent être questionnés les liens que le numérique a avec la démocratie. Ici déjà, il conviendra de s'accorder sur les termes, numérique ou outil numérique ou internet seront considérés comme synonymes.

Un autre préalable conduira à adopter, selon $\mathrm{H}$. Kelsen, une définition normative de la démocratie en considérant que le peuple est constitué par l'ensemble des individus destinataires de la norme. Dès lors, la démocratie se définit comme l'«identité du sujet et de l'objet du pouvoir, des gouvernants et des gouvernés $»^{3}$. Ainsi se justifie la participation du citoyen au pouvoir décisionnaire. Ce faisant, la question se pose de savoir comment les gouvernés exercent leur pouvoir ou plus exactement comment les citoyens sont associés à l'exercice du pouvoir. Différents systèmes de participation sont identifiés et celui de la démocratie représentative dans lequel le corps électoral désigne et les représentants décident, est celui qui recueille la plus grande adhésion. En effet devant l'impossibilité matérielle de la démocratie directe eu égard aux dimensions de l'État moderne, le principe de la représentation apparaît comme un mécanisme de bon sens (il existe également et bien évidemment des raisons politiques au-delà des simples raisons pratiques). En outre, selon Sieyès, la représentation a pour seule fonction de donner un contenu unitaire à la volonté nationale, peu importe l'existence de diversités au sein du peuple et des citoyens car «les avantages par lesquels [ils] diffèrent entre eux sont au-delà du caractère du citoyen $»^{4}$. La doctrine classique, sur ces fondements, posera les bases de la démocratie représentative en considérant le peuple en tant que corps électoral détenteur du pouvoir de suffrage qu'Hauriou définit comme «l'organisation de l'assentiment $»^{5}$. Le droit de suffrage attaché à chaque citoyen de manière égale complète le dispositif en excluant de toute considération l'existence de différences, spécificités ou autres distinctions au sein du peuple

\footnotetext{
1 Maître de conférences HDR, Aix-Marseille Univ., Université de Toulon, Univ. Pau \& Pays Adour, CNRS, DICE, ILF-GERJC, Aix-enProvence, France.

2 P. Rosanvallon, La contre-démocratie, la politique à l'âge de la défiance, Seuil, 2006, 345 p. et La Légitimité démocratique, Seuil, Points Essais, 2008, 368 p.

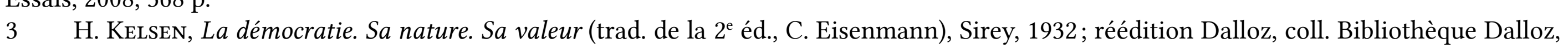
2004, p. 14.

4 Qu'est-ce que le Tiers-état?

5 M. Hauriou, Précis de droit constitutionnel, Paris, Sirey, 2è, éd., 1929, rééd. Ed. du CNRS, 1965, p. 544.
} 
et en considérant la nation et le peuple comme un tout homogène. Cette approche de la démocratie représentative associée au libéralisme politique fonde la nouvelle légitimité politique apparue au XVIII ${ }^{\mathrm{e}}$ siècle et se perpétue, même après la Première Guerre mondiale.

Dans ce contexte, la technique mise en œuvre, la plus fréquemment utilisée pour associer les citoyens au pouvoir et donc pour désigner les gouvernants, est l'élection, laquelle se concrétise à travers les règles et principes organisés au sein du droit électoral. Ce dernier est l'instrument privilégié du respect de la démocratie en posant les principes qui la garantissent. Or les deux principes cardinaux garantissant une élection démocratique sont la liberté et l'égalité ou encore le respect de l'égalité entre les citoyens et l'expression libre de leurs opinions tout au long du processus électoral. Celui-ci se concrétise non seulement lors du jour du scrutin mais également englobe, d'une part, en amont du scrutin, la campagne électorale portée par des candidats dûment déclarés et, d'autre part, en aval du scrutin, l'intervention, éventuelle, du juge électoral dont la mission précisément est d'assurer la régularité et la sincérité du scrutin, soit de vérifier que les principes de liberté et d'égalité ont été respectés et que les résultats sont conformes aux souhaits des citoyens, le secret du vote constituant, in fine, un troisième principe nécessaire.

Mais face à ces principes de l'élection au sein d'une démocratie représentative, exposés succinctement, on ne peut que constater l'existence d'un fossé grandissant entre les citoyens et les élites politiques: faible représentativité des élus, abstention et refus de voter, incapacité supposée de la part des élites à réformer un système en déliquescence. En résumé, c'est à un échec de la démocratie traditionnellement définie que l'on aboutirait.

Parallèlement le besoin d'horizontalité de nos sociétés contemporaines se fait jour. Celui lié à la transparence, à la proximité ou à la participation directe se manifeste de plus en plus. Le numérique semble apporter des réponses : l'économie collaborative, la mise en commun d'informations de toute nature sur les sites internet dédiés, les blogs ou via les réseaux sociaux ainsi que la transmission facilitée de ces dernières par des instruments détenus par nombre d'individus sur la planète transforment inévitablement nos organisations sociales. Il n'est donc pas étonnant que la démocratie et les modalités de son exercice soient elles aussi impactées par cette troisième révolution industrielle, voire, même, qu'elles puissent la régénérer ou la refonder: «Au début des années 1990, alors qu'internet commence à sortir du monde universitaire où il est né, les chantres de cette nouvelle technique y voient un dispositif capable de revitaliser la démocratie, un espace public accessible à tous qui permettrait aux citoyens non seulement de débattre des grandes questions politiques, mais aussi de s'inscrire dans le processus délibératif $»^{6}$. Internet devient ainsi un vecteur mais également un lieu de démocratie participative, délibérative mais encore du discours, de l'échange ou de l'engagement politiques favorisant la démocratie 2.0. En témoignent les réflexions portées pour le Forum mondial de la démocratie ${ }^{7}$ depuis 2012 à l'initiative du conseil de l'Europe dont les thématiques embrassent

\footnotetext{
6 P. Flichy, «LA DÉMOCRATIE $2.0 »$, Études, vol. tome 412, nº. 5, 2010, p. 617-626.

Cf. www.coe.int/fr/web/world-forum-democracy/home: Le Forum mondial de la Démocratie est une plateforme unique qui permet aux décideurs et aux militants de débattre de solutions aux défis qui se posent à nos démocraties. En identifiant et en analysant des initiatives et pratiques expérimentales, le Forum met en avant et encourage des innovations démocratiques provenant de la base et leur transfert à un
} 
régulièrement la démocratie 2.0 ; ou encore l'organisation d'une participation politique et citoyenne en ligne à partir des «Civic Tech» dont l'objectif est de doter la démocratie représentative actuelle d'un volet participatif.

Dès lors, l'organisation du processus électoral ne peut être qu'affectée par les outils numériques et les nouvelles technologies de l'information et de la communication. Internet modifie ou redéfinit les conceptions traditionnelles de l'élection tant dans son approche collective qu'individuelle sous réserve que l'ensemble des acteurs dispose d'un accès à cet outil. Est-ce que pour autant les garanties démocratiques sont affectées par l'utilisation d'internet? Ou pour le dire autrement existe-t-il un impact du numérique sur les principes de liberté et d'égalité qui conditionnent le déroulement d'une élection? Plus précisément, la sincérité du scrutin serait-elle compromise par ces nouvelles pratiques?

La réponse est mitigée. D'abord parce que s'il est certain que l'arrivée d'internet bouleverse les pratiques, seules des études notamment de nature sociologique et menées sur plusieurs types d'élections successives permettraient d'obtenir certainement une vision des pratiques et des usages plus fine. Ensuite, il ne faut peut-être pas surestimer les vertus de la démocratie 2.0 (ou 3.0!) parce que des dangers liés notamment à la protection des données privées ou aux algorithmes de recommandation sont à l'œuvre. En effet au plan collectif, dans le cadre du vote électronique, comment garantir l'égalité de décompte des voix, à savoir s'assurer que le résultat de l'élection est fidèle à la volonté de la majorité réelle du corps électoral ou selon la formule consacrée, qu'un électeur égale une voix? S'agissant de l'électeur considéré individuellement, sa liberté de vote se concrétise par le caractère secret du vote. Or, comment garantir un tel caractère fondamental avec le vote électronique ou le vote à distance ou, avec de tels outils, comment garantir que le citoyen émet un vote clair, non faussé par les pressions et mieux encore la fraude? Enfin, il est peu vraisemblable, même si la technologie évolue rapidement, que les modes et systèmes d'une démocratie représentative, participative ou encore directe soient en totalité remplacés par un outil numérique. Ce serait éliminer le facteur humain et quasi charnel qui jusqu'à présent singularise le processus électoral et le lien existant entre un candidat et ses électeurs plus spécifiquement dans le cadre d'élections présidentielles ou municipales.

Ainsi, en l'état du droit des élections, tel qu'il existe et tel que le juge l'adapte, on constatera que les garanties existent. La réglementation qui pose les principes (I) et le juge qui les interprète (II) en utilisant les techniques propres au contentieux électoral apparaissent compatibles avec l'immixtion du numérique dans le processus électoral. On précisera d'une part, que le cadre défini est celui de l'élection et de la démocratie représentative et non celui de la démocratie dite participative qui s'intéresse davantage aux mécanismes délibératifs et non décisionnaires; et, d'autre part qu'il ne s'agit pas de manifester une hostilité quelconque à l'égard de l'irruption des outils numériques dans le processus électoral mais plutôt de tenter de vérifier que les garanties posées depuis la mise

niveau systémique afin de renforcer les fondations des sociétés démocratiques. Le Forum contribue ainsi à faire évoluer la démocratie vers des structures et des institutions plus participatives et inclusives. 
en œuvre de ces dispositifs sont concevables en dépit des nouvelles pratiques. Leur objectif initial, celui d'assurer la sincérité du scrutin demeure toujours la préoccupation du juge électoral.

\section{Droit des élections et numérique : une réglementation adaptée}

Il est vrai qu'internet et plus généralement les nouvelles technologies de l'information et de la communication ne font pas l'objet de titres, chapitres ou sections spécifiques au sein du Code électoral avec des intitulés directement dédiés. ${ }^{8}$. Mais ce n'est pas parce que ce droit ne fait pas de mention expresse à ces nouveaux instruments qu'il est dans l'incapacité de les organiser et d'en appréhender les effets. On peut dater l'utilisation de ces moyens électroniques des élections municipales et cantonales de 2001 avec une consécration lors de la campagne électorale lors des élections présidentielles de 2007. C'est précisément à l'occasion d'un contentieux portant sur les élections municipales de Rodez dès 2002 que le rapporteur public précisait qu'il n'y avait pas de «vide juridique » sur internet « car il n’y a aucune raison que les règles générales du droit pénal, du droit civil, fiscal et commercial ne lui soient pas applicables. En d'autres termes, internet n'est pas une terre de "non-droit" ou d'extraterritorialité juridique; par ailleurs, la plupart des États ont jugé opportun de ne pas élaborer une législation internet spécifique, et ont préféré laisser aux juges le soin d'appliquer de façon adaptée à l'état des techniques - peu fixé en ce domaine - les règles du droit commun » ${ }^{9}$. C'est en effet cette voie qui est préférée.

S'agissant du processus électoral, le domaine dans lequel l'impact du numérique bouleverse les pratiques est celui de la campagne électorale. Les dispositifs élaborés intéressent tout à la fois la propagande électorale et le financement de la campagne. Dans tous les cas, ces règles sont présentes afin de garantir que le citoyen puisse exprimer son opinion librement, qu'il ne soit pas l'objet de pression (ce qui implique la neutralité de l'autorité administrative et la loyauté des procédés utilisés) et que son vote demeure secret, le tout dans le respect de l'égalité, conséquence de l'universalité du suffrage, et que les résultats électoraux correspondent à la majorité qui s'est réellement exprimée. Ce sont les objectifs de la démocratie et par voie de conséquence ce sont ceux que s'assigne le droit des élections.

Les différentes règles organisant la communication des candidats ou celle des collectivités territoriales en période préélectorale ou électorale sont identifiées dans le code électoral aux articles L. 47 à L. 52-3 pour les élections des députés ${ }^{10}$, des conseillers départementaux et municipaux. Les règles relatives à l'élection présidentielle sont éparpillées dans la Constitution (articles 6 et 7), la loi

\footnotetext{
8 En matière de campagne électorale, le code électoral ne fait référence au NTIC qu'aux articles L 48 -1 et L 49 issus de la loi n $2011-412$ du 14 avril 2011 portant simplification de dispositions du code électoral et relative à la transparence financière de la vie politique.

9 CE, 8 juillet 2002, Élections municipales de Rodez, req. 239220, Leb. T. 746; Conclusions I. de Silva, BfCL, nº 7/02, déc. 2002, p. 508, «Internet échappe-t-il aux règles classiques de propagande électorale? ».

10 La campagne pour les élections législatives est très encadrée en raison des modalités de son financement. En revanche, eu égard à son caractère moins dispendieux, dû au faible nombre des grands électeurs composant le collège électoral sénatorial, la campagne pour les élections sénatoriales est pour sa part soumise à des règles plus souples. Les articles L 306 à L 308-1 du Code électoral posent succinctement les conditions relatives aux réunions électorales: elles peuvent se dérouler au cours des six semaines précédant le scrutin. Elles sont réservées aux membres du collège électoral et à leurs suppléants. Il n'y a pas d'affichage public. Depuis la loi organique du 14 avril 2011 , les règles relatives au financement des campagnes électorales (plafonnement des dépenses, désignation d'un mandataire financier, ouverture d'un compte réservé à la campagne et dépôt du compte à la commission nationale des comptes de campagne et des financements politiques) sont applicables aux sénateurs.
} 
organique (ordonnance $\mathrm{n}^{\circ}$ 58-1067 du 7 novembre 1958 sur le CC et dispositions organiques de la loi référendaire nº2-1292 du 6 novembre 1962 et ses modifications successives) et le décret $\mathrm{n}^{\circ}$ 2001-213 du 8 mars 2001 portant application de la loi nº62-1292 du 6 novembre 1962 relative à l'élection du Président de la République au suffrage universel. À l'ensemble de ces textes spécifiques aux élections, s'ajoutent ceux organisant la communication audiovisuelle (la loi du 30 septembre 1986 précise le principe de la liberté de communication), les sondages (la loi n ${ }^{\circ} 77-808$ du 19 juillet 1977 est relative à la publication et à la diffusion des sondages) et la protection des données personnelles (loi du 6 janvier 1978 modifiée, dite loi «Informatique et Libertés»). Chacun de ces domaines trouve un champ d'application spécifique dans le cadre des élections et les autorités administratives indépendantes - le Conseil supérieur de l'audiovisuel et la Commission nationale de l'informatique et des libertés - et autorité publique indépendante - la Commission des sondages - participent activement à l'encadrement de chacun des scrutins. Il s'agit successivement de faire respecter le pluralisme dans les médias audiovisuels, d'assurer la transparence des opérations de sondages ou de protéger le droit au respect de la vie privée et le respect des données personnelles. Enfin, chaque élection fait l'objet de textes permanents (instructions du Ministère de l'Intérieur) ou ponctuels pour l'organisation de tel ou tel scrutin.

À ces règles s'ajoutent celles relatives au financement des campagnes électorales et les sanctions découlant de son non-respect. Désormais parmi les moyens soulevés dans une requête à l'encontre d'un résultat de scrutin, peuvent être mis en avant des éléments relatifs au mode de financement de la campagne. Les moyens classiques relevant de faits de propagande peuvent ainsi s'enrichir de moyens relatifs aux dépenses ou recettes du candidat, à la présentation de son compte de campagne, etc. Le code électoral ${ }^{11}$ dispose d'un ensemble de règles permettant d'assurer la transparence du processus électoral par l'encadrement des modalités de financement des campagnes électorales. Les dépenses dans la quasi-totalité des élections ${ }^{12}$ sont plafonnées, elles sont retracées dans un compte de campagne certifié par un expert-comptable. Le compte de campagne détaille également toutes les recettes du candidat qui doivent être recueillies par un intermédiaire financier, le mandataire ou l'association de financement. Les dons de personnes morales autres que ceux d'un parti politique sont interdits. Ce compte doit être déposé auprès de la Commission nationale des comptes de campagne et des financements politiques (CNCCFP) dans le délai de deux mois. Enfin des sanctions spécifiques accompagnent le dispositif. Ainsi la réglementation touchant aux moyens de propagande dans le cadre de la compétition électorale se double-t-elle d'un dispositif organisant ses modalités de financement. Dans les deux cas, les principes qui animent le déroulement de la campagne électorale et de son financement sont intangibles.

11 Les articles du code électoral concernés par le financement des campagnes électorales sont: articles L52-4 à L52-18, articles L118-2 et L118-3, article L308-1, articles LO128, LO 136-1, LO186-1 et article 3 de la loi nº6-1292 du 6 novembre 1962.

12 Tous les candidats présents aux élections européennes, législatives, régionales, cantonales, municipales (à l'exception des communes et cantons de moins de 9000 habitants) sont concernés par ces dispositions. Les élections sénatoriales ne sont soumises qu'à l'interdiction de financement des campagnes par des personnes morales. La loi nº62-1292 du 6 novembre 1962 (article 3-II) assujettit l'élection du Président de la République aux dispositions du Code électoral relatives au financement de la campagne électorale. Pour la première fois en 2007, l'examen des comptes de campagne des candidats à l'élection présidentielle appartient à la CNCCFP. Antérieurement, la compétence du Conseil constitutionnel était exclusive sur le contrôle de ces comptes de campagne. 
Ce corpus textuel ${ }^{13}$ est dense, relève de plusieurs sources normatives et implique toutes les dimensions constituant le cœur d'une élection et ses périphéries. Mais tous ces textes concourent à garantir les principes cardinaux du droit des élections. Leur particularité, notamment concernant le code électoral est de ne pas véritablement distinguer entre les supports potentiels utilisés. Si seuls les articles L 48-1 et L 49 du code électoral envisagent modestement la communication par voie électronique, le code électoral et, globalement, l'ensemble des textes cités s'intéressent principalement aux contenus et aux effets de la communication sur les électeurs. C'est pourquoi l'utilisation de moyens de propagande ou de communication via internet et les technologies du numérique est pleinement compatible avec la réglementation électorale. De surcroît, la jurisprudence, abondante, permet une constante adaptation et l'on vérifiera ci-après que les techniques et principes sont, là encore, pleinement compatibles avec l'introduction de ces nouvelles modalités.

Dès lors, si le contenu est prépondérant par rapport au vecteur le diffusant, quel qu'il soit, ce dernier est pleinement assujetti à l'ensemble des règles qui organisent une campagne électorale. Brièvement, celle-ci est surtout conditionnée par l'aspect temporel car, sans décrire de manière exhaustive l'ensemble des règles de la campagne, on rappellera que si la liberté d'expression est la règle, les restrictions à cette liberté augmentent au fur et à mesure que l'on se rapproche du jour du scrutin. Les dispositifs existants distinguent entre les différents types de contenus émis au cours d'une campagne. En effet, le calendrier électoral se découpe en différentes périodes : les six mois précédant l'élection, la veille et le jour du scrutin. Le premier jour du sixième mois précédant le premier jour du mois de l'élection et courant jusqu'à la date de dépôt du compte de campagne, ouvre la période de financement de la campagne électorale (article L. 52-4) avec l'interdiction de financement par toute personne morale de droit public comme de droit privé, à l'exception des partis ou groupements politiques, de la campagne d'un candidat (article L. 52-8); lors de cette période, l'utilisation des moyens des collectivités territoriales à des fins électorales (article L. 52-1 alinéa 2) est prohibée et quant au candidat, de nouvelles restrictions sont ajoutées: interdiction de mettre en place un système gratuit de téléphonie au profit du candidat (article L. 50-1), interdiction de l'affichage hors des emplacements prévus par l'autorité municipale (article L. 51), interdiction de la publicité commerciale (article L. 52-1 alinéa 1). À la veille du scrutin les restrictions se renforcent et tout message à des fins de propagande est interdit (article L. 49). La liberté d'expression des candidats est mise entre parenthèses le jour du scrutin afin de ne pas porter atteinte au bon déroulement des opérations de vote. Dans tous les cas mentionnés, le support numérique est concerné. Le juge a pris en compte cette nouveauté en vérifiant, comme pour toutes les autres modalités de communication pendant la campagne électorale, que les réseaux sociaux, les blogs, les sites internet des candidats, des partis, des tiers et tout ce qui transite par un support numérique respectent les principes posés tant en termes

13 On notera que d'autres types de règles que l'on peut qualifier de droit souple apparaissent également dans le corpus des règles applicables. En effet de nombreuses recommandations et/ou guides pratiques à destination des candidats sont édictés notamment par la CNIL, le CSA ou la CNCCFP. 
de propagande que dans les modalités de financement. Le juge use des mêmes méthodes sans se départir dans ses modalités d'examen du contenu du message de son pragmatisme habituel ${ }^{14}$.

\section{Contentieux électoral et numérique : une sincérité du scrutin à préserver}

En 2002, la Revue, les Cahiers du Conseil constitutionnel ${ }^{15}$, avait accueilli dans ses pages une étude relative à la sincérité du scrutin et portée par le Professeur Richard Ghevontian. En 2010, un colloque organisé par l'Institut Louis Favoreu était consacré à «Sincérité et démocratie ». Les travaux réalisés ont permis de mettre en évidence le caractère central et continu de la notion de sincérité au sein du contentieux électoral mais également les transformations auxquelles elle est confrontée. L'utilisation du numérique dans le processus électoral et donc, le contentieux susceptible d'en ressortir, en font partie.

Le juge électoral utilise très fréquemment la notion de sincérité dans ses décisions et lui fait jouer un rôle majeur puisque c'est son respect ou son atteinte qui détermine, le plus souvent, le sort du contentieux en cours. Dès lors, une élection sera régulière si sa sincérité est garantie c'est-à-dire s'il y a adéquation entre le résultat proclamé et la volonté majoritaire librement exprimée. La sincérité du scrutin et sa recherche par le juge deviennent le révélateur de la volonté réelle de l'électeur. Si elle ne peut être connue de manière certaine et donc s'il est impossible de connaître avec certitude le choix majoritaire des électeurs, alors l'élection est annulée. Néanmoins le terme même de sincérité n'est pas juridique, il relève de la morale. Et pourtant c'est sur la question de savoir si l'élection qui vient de se dérouler a été acquise de manière sincère que repose tout le processus démocratique. Or, dans la mesure où la démocratie - dont l'élection constitue le premier mode d'expression - est le seul régime politique compatible avec l'État de droit, la recherche de la sincérité du scrutin, la recherche de la vérité, devient essentielle. C'est là que réside la mission du juge électoral. Celle-ci diffère des finalités traditionnelles d'un juge dans un autre type de contentieux (rectification de la légalité ou réparation d'un préjudice).

Mais la sincérité trouve également ses fondements au sein des principes fondamentaux du droit électoral largement partagés au plan européen ${ }^{16}$. On peut ici reprendre l'expression de Pierre Garrone de «patrimoine électoral européen » car ces principes sont présents dans la plupart des Constitutions des États se réclamant d'un État de droit. La Constitution française de 1958 les souligne à l'article 3 et les textes européens relatifs aux droits fondamentaux y font référence (Protocole additionnel n ${ }^{\circ} 11$ à la Convention européenne de sauvegarde des droits de l'homme, article 3; Charte des droits fondamentaux de l'Union européenne, article 39 alinéa 2). Dès lors, les principes permettant de garantir

\footnotetext{
14 Sur différents usages de l'outil numérique pendant la campagne électorale voir S. DEFIX, «Réseaux sociaux et règles de propagande électorale », A7CT, décembre 2014, p. 580-584; G. PrUNIER, «Outils numériques et campagne électorale. Spécificités des règles applicables aux nouvelles technologies », AfCT, juin 2013, p. 283-287; J.-M. NoTARIANNI, « Droit électoral et technologie de l'information et de la communication », $R G C T, \mathrm{n}^{\circ} 42$, octobre-novembre-décembre 2007, p. 235-257.

15 CCC, Études et doctrine, $\mathrm{n}^{\circ}$ 13/2002, p. 61-103.

16 P. GARRone, «Le patrimoine électoral européen. Une décennie d'expérience de la Commission de Venise dans le domaine électoral», $R D P, 2001, \mathrm{n}^{\circ} 5$, p. $1417-1454$.
} 
cette sincérité sont l'égalité, la liberté, le caractère secret du vote ${ }^{17}$. En outre, parmi les techniques du juge électoral, deux paramètres éclairent l'atteinte ou non à la sincérité du scrutin : l'écart de voix et l'influence déterminante de l'irrégularité génératrice du défaut de sincérité.

Muni de ces instruments, le juge examine l'atteinte à la sincérité sous l'angle collectif, c'est-à-dire du corps électoral dans son ensemble, en mobilisant les principes d'égalité et de liberté. S'agissant de l'égalité, il vérifie que l'égalité des conditions de la compétition (toutes les candidatures sont soumises au même traitement) est remplie, que l'égalité de décompte des voix (chaque électeur dispose du même poids que les autres) est assurée ou encore, que l'égalité de représentation (technique du découpage électoral) est respectée. Concernant le principe de liberté, il convient de vérifier que les électeurs sont à l'abri de la pression de l'autorité publique, soit de sa neutralité, intervenant tant au titre d'institution politique qu'à celui d'autorité administrative notamment dans la campagne électorale. Le juge examine également l'atteinte à la sincérité sous l'angle individuel, celui de l'électeur. À cette fin c'est le caractère secret du vote qui doit être préservé pour que l'électeur exerce son choix en toute liberté, en toute conscience et en suivant sa seule volonté, et donc, à l'abri des pressions. En effet la sincérité du scrutin ne peut être attestée que si l'on peut connaître clairement le choix et donc la volonté de l'électeur. La clarté du choix de l'électeur dépendra ainsi de la vérification des suffrages exprimés corrélés à l'existence ou non de fraudes électorales et de leur influence ou non sur le résultat (utilisation de la théorie de l'influence déterminante).

Ainsi le contentieux électoral témoigne-t-il, avec la recherche par le juge de la préservation de la sincérité du scrutin, d'une singularité. En outre le processus électoral est complexe. Il ne se réduit pas au seul jour du scrutin mais englobe les opérations situées en aval de ce dernier (déclaration de candidature, inscription sur les listes électorales, campagne électorale, financement...) et celles apparaissant en amont (inéligibilités...). Dès lors le contentieux est extrêmement diversifié et le juge traitant des élections se présente sous différentes formes: le contentieux de la liste électorale appartient au juge judiciaire, celui des élections locales au juge administratif et enfin celui des votations nationales au Conseil constitutionnel. Quoi qu'il en soit, son objectif est toujours le même: s'assurer de la sincérité du scrutin afin que ceux qui ont été proclamés élus sont bel et bien ceux qui correspondent à la volonté des électeurs. Dès lors il vérifie seulement que les conditions d'une régularité élémentaire ont été respectées au plan externe (est-ce que les formes, les procédures ont été respectées?) au plan interne (est-ce que les résultats sont valides?). En aucun cas il ne se substitue au verdict du peuple, il ne peut pas se transformer en service d'enregistrement des candidatures, en commission électorale, en bureau électoral etc., bref il ne peut pas rejouer l'ensemble des étapes du processus électoral.

Dans ce contexte, l'introduction du numérique n'est pas un obstacle et la souplesse des instruments du contentieux électoral permet, au surplus, de tenir compte de cette transformation dans le processus électoral. En outre la nature même du contentieux électoral permet d'envisager les

17 Pour identifier les liens entre la notion de sincérité et le processus de l'élection au regard des principes énoncés, voir R. GHEvontiAN, «La notion de sincérité du scrutin», CCC, nº 13/2002, p. 63-68. 
nouveautés, quelles qu'elles soient, sans doute avec vigilance mais également avec sérénité. En effet, pour regrettable que cela soit dans le cadre d'une approche théorique, le contentieux électoral n'est pas aisément classifiable car il révèle un enjeu démocratique. Dans la dichotomie traditionnelle et de façon schématique, le contentieux objectif est un procès fait à l'acte dans le but de rétablir sa légalité ou sa constitutionnalité et le contentieux subjectif se caractérise par une réparation pour compenser la violation d'un droit subjectif. Or le contentieux électoral recèle les deux aspects: dans le processus électoral, un acte administratif peut par exemple être entaché d'illégalité (acte de candidature, acte de sectionnement électoral, acte de proclamation des résultats...) sans pour autant être annulé et, en même temps ce sont les droits politiques des élus ou des candidats ou des électeurs qui sont en cause c'est-à-dire ceux qui permettent de s'exprimer et de participer à la vie en société (au sens strict le droit de vote et le droit d'être candidat, au sens large le droit de manifester, les droits civiques, la liberté association, la liberté d'expression, la liberté d'information, la liberté de réunion). On comprend donc que la classification du contentieux électoral dans l'une ou l'autre des deux grandes familles soit sinon impossible du moins difficile ${ }^{18}$ ! Mais cela facilite le changement tout en préservant les principes garantissant le processus électif et, en définitive, la démocratie représentative. En revanche, si la nature de nos modes contemporains d'organisation du gouvernement venait à changer radicalement, assurément, lesdits principes, garanties et autres techniques contentieuses deviendraient obsolètes. Aujourd'hui, la démocratie est la seule forme de pouvoir compatible avec l'État de droit. Elle ne peut réellement exister que si l'élection, qui en constitue le temps fort, est entourée de toutes les garanties nécessaires pour en assurer la régularité et la sincérité. Liberté, égalité et leurs différentes déclinaisons demeurent les piliers sur lesquels elle se bâtit quotidiennement en s'adaptant à toutes les nouveautés qui enrichissent nos sociétés contemporaines.

18 Pour une présentation synthétique des réflexions sur la place du contentieux électoral au sein du contentieux administratif voir $\mathrm{S}$. ToRCOL, «Le contentieux des élections législatives : réflexions autour d'un contentieux à risques », RDP, 5-2004, p. 1211-1241, spéc. p. 1215-1217. 



\title{
NumériQue : activisme et influence politiQue
}

\author{
François-Bernard HuYGHE ${ }^{1}$
}

Dans les années 1990, les premières anticipations sur le devenir d'internet se construisent autour de trois mythes fondateurs:

- celui du contrôle absolu à la Big Brother: les techniques instruments de surveillance du citoyen tracé, observé, espionné, prévisible et conforme;

- celui du chaos : le grand accident informatique ou le grand sabotage plongeant dans l'anarchie nos systèmes économiques et politiques dépendants de l'informatique;

- celui de la prise de pouvoir démocratique: ne pouvant plus être isolés ou censurés, désormais capables de s'exprimer ou de participer à la vie publique, les citoyens allaient inventer de nouvelles formes de démocratie sur l'Agora planétaire qu'ouvrirait le cybermonde.

Deux ans après une ébauche de «Twitter révolution » iranienne manquée, c'est ce dernier thème qu'a semblé illustrer le printemps arabe de 2011 d'abord en Tunisie puis en Égypte, avec des révoltes ou révolutions vite baptisées «Facebook» ou « $2.0 »$ par leurs propres partisans. Des révoltes que tentent d'ailleurs vite d'imiter des manifestants d'autres pays après qu'elles se soient répandues dans le monde arabe comme par mimétisme d'un activisme inédit.

Bien sûr, personne ne soutient sérieusement que les printemps arabes aient donné raison seuls au cyberutopisme militant. Si les révolutions politiques ne dépendaient que de conditions technologiques, ce serait trop simple. Ce serait négliger des disparités (voir le taux d'équipement internet très différent de la Tunisie à la Libye), sous-estimer le rôle des télévisions par satellites arabophones et la mobilisation de l'opinion libérale internationale. Ce serait surtout oublier qu'une révolution est un acte de confrontation physique par lequel des foules s'emparent d'un pouvoir face à une police qui tire ou ne tire pas. Ce serait enfin ignorer que l'efficacité des réseaux sociaux pour « dégager » un pouvoir discrédité ne garantit ni une fin heureuse, ni une victoire électorale, ni ne protège contre le retour des «vieux» coups d'État ou de la violence djihadiste.

Mais, même réduit à un rôle d'accélérateur, d'amplificateur ou de déclencheur d'événements qui ont d'autres causes et impliquent d'autres processus, internet a changé la façon de militer, de se mobiliser voire d'être ensemble et de faire de la politique.

$1 \quad$ Directeur de recherche à l'IRIS. 
Les États le savent et recherchent de nouvelles stratégies de censure, ou veulent tourner à leur profit le phénomène des réseaux sociaux, qu'il s'agisse de combattre leurs adversaires politiques ou d'amplifier une influence, déjà relayée par des médias classiques et des réseaux humains...

Mais le jeu s'ouvre à d'autres acteurs encore : groupes de hackers professant parfois une idéologie de la transparence hostile à tout pouvoir, éventuellement groupes mercenaires à faux drapeaux, acteurs économiques qui ont des intérêts financiers évidents dans leur conquête des marchés mais qui se trouvent confrontés à des questions d'éthique et de politique relatives au contrôle d'internet (voir l'exemple du bras de fer entre la Chine et Google ou Facebook rétropédalant pour assurer qu'il combattra la diffusion de fausses nouvelles susceptibles notamment d'influer sur les élections).

Les réseaux sociaux se sont montrés extraordinairement efficaces pour exprimer des opinions interdites, pour stimuler des indignations contagieuses, pour trouver des appuis hors frontières (médias, diaspora, groupes militants tels les Anonymous...), pour montrer les exactions du pouvoir, pour donner des mots d'ordre de rassemblement ou énoncer des revendications et pour jouer à cachecache avec la police.

Leurs atouts :

- vitesse de communication et de mouvement supérieure à celle de l'adversaire débordé,

- initiative constante et prise de décision décentralisée bien en accord avec la structure des réseaux,

- recours à «l'intelligence des foules», des solutions élaborées collectivement, que ce soit pour contrer les initiatives policières ou imaginer des contre-discours,

- faculté de faire converger des indignations diffuses en mobilisations contre un objectif symbolique (l'autocrate à qui on crie « dégage » ou le système),

- capacité éventuellement de converger physiquement de partout vers un objectif unique comme une sorte d'essaim, de «swarming» suivant la terminologie américaine,

- volonté de déborder les frontières nationales pour trouver un écho, des alliés ou des ressources techniques,

- théâtralité, sens de l'image proposée aux médias (une révolte populaire, sans chefs, directe, sans médiations ni partis, représentative de la société civile, n'ayant besoin ni d'avant-gardes ni de violence pour parvenir à ses fins) : autant de leçons stratégiques à retenir de ces affrontements.

Le «faible» (la foule coordonnée par les technologies de l'information) menace le «fort» (l'État). Ce dernier ne peut plus exercer ses attributs classiques: le monopole de la violence légitime (qui arrêter?), la territorialité (les électrons ignorent les barrières douanières et l'État doit combattre des 
cyberadversaires ou cyberdissidents hors frontières), l'autorité (débordée par des foules plus rapides, plus coordonnées...) et même le spectacle du pouvoir (le discours officiel n'attire même plus l'attention des populations qui se tournent, soit vers le web, soit vers les télévisions internationales d'information).

Les réseaux sociaux ne servent pas seulement à publier des opinions qui seraient autrement étouffées ou à faire des révélations qui seraient censurées: ils font naître de nouvelles formes de communautés et de faire passer d'un lien «faible» dans le cybermonde à un lien fort et passionnel dans la «vraie vie», comme ils commencent à créer l'ébauche d'un espace public pour une société civile sous le boisseau. Sans oublier qu'ils servent aussi à provoquer symboliquement un pouvoir (en attendant de le défier physiquement par des occupations interdites des places et des rues).

Les autocraties sont parfois capables de réagir: elles vont utiliser de nouveaux instruments sur la Toile, plus subtils que les arrestations de blogueurs influents ou la coupure du Net (intenable plus de quelques heures). Adopter de fausses identités pour infiltrer la cyberdissidence, créer des réseaux de blogueurs "patriotes à la chinoise », se doter d'équivalents nationaux de Facebook ou de Google, et d'une blogosphère sous contrôle, peser sur les fournisseurs d'accès, exploiter des mots-clefs pour attirer les recherches sur des sites souhaités comme en Syrie, attaquer des sites étrangers qui aident la contestation, utiliser de la technologie occidentale pour filtrer et repérer en attendant de se doter d'un internet «balkanisé », avoir le contrôle sur ses propres fournisseurs d'accès, ses moteurs de recherche et ses réseaux, «géolocaliser » les adversaires en mouvement, analyser les réseaux sociaux et leurs échanges pour prédire qui risque de rentrer dans la dissidence (comme les sociétés commerciales «prédisent» l'intérêt de tel internaute pour tel type de produit): telles sont les ripostes qui se dessinent sous nos yeux. Et il n'est nullement certain qu'elles soient partout inefficaces.

L'État peut aussi espérer gagner une influence au-delà de ses frontières grâce à ces mêmes technologies 2.0, ou du moins déstabiliser des rivaux en encourageant des mouvements d'opinion.

Le politique peut exercer une contrainte ou un contrôle, passer des alliances ou entamer une confrontation avec des acteurs économiques et techniques - opérateurs, fournisseurs d'accès, services de connexion - pour faciliter ou écraser tel courant d'opinion. Il peut aussi imiter les techniques du «faible». Par exemple en déstabilisant un État ou un mouvement d'opinion adverse par des «leaks», de fuites, qui combinent la méthode du hacker pour accéder aux documents compromettants, la tactique de l'activiste pour leur donner un écho international et une stratégie globale de guerre de l'information.

Comme on le voit, les «coups» que peuvent jouer les acteurs - activistes, bureaucraties, organisations non-étatiques, entreprises - sont nombreux, complexes et souvent entourés d'un halo de secret. 
À cette complexité technique et stratégique, il faut ajouter un élément idéologique au sens le plus large : le poids de ce que l'on croit vrai en fonction de ses valeurs et de l'opposition à un adversaire politique. L'élection présidentielle américaine de 2016 - l'année où «ère de la post-vérité »a été promu mot de l'année par les Oxford Dictionnaries - a montré une double rhétorique de défense promue notamment par l'administration Obama et la majorité des médias mainstream américains:

- Premier élément: l'hypothèse d'une intervention russe pour fausser le processus démocratique. Celle-ci aurait pris la quadruple forme d'intrusions informatiques (hacking, utilisation de trolls pour polluer les débats), de mobilisation de réseaux humains complices (dont ceux de Trump lui-même), de l'utilisation de médias internationaux de propagande (comme Rossia Today ou Radio Spoutnik qui feraient dans le sens Est-Ouest ce que Voice of America et Radio Free Europe faisaient dans le sens Ouest Est pendant la guerre froide) et enfin de la diffusion de «fakes», de fausses nouvelles déstabilisatrices et subversives sur les réseaux sociaux.

- Second facteur: la réceptivité du public à ces «fakes» justement qui auraient largement déterminé le vote à partir de données ou de rumeurs volontairement biaisées. Que l'on explique cela par l'habileté des faussaires en ligne, par la crédulité du public envers tout ce qui flatte ses préjugés et qui le rend imperméable à l'information mainstream ou encore par un effet pervers: les réseaux sociaux enferment dans des «bulles d'isolement» favorables par nature à la propagation d'une information douteuse «de pair à pair» et au développement d'interprétations délirantes et théories complotistes.

Du coup on voit se mobiliser des gouvernements, de médias, mais aussi des grandes compagnies du Net pour repérer, signaler, vérifier, réfuter et éventuellement rendre inaccessibles aux tentatives de recherches des contenus faux, relevant d'opérations d'influence ou de discours de haine.

Ils réagissent comme si une allergie des masses au réel menaçait nos démocraties. Le discours d'en haut, censé s'appuyer sur la science ou des faits, est concurrencé par des réalités alternatives conformes aux passions et aux peurs des masses; ces dernières se détournent des mass médias au profit des «révélations» des «communautés» virtuelles. Si l'on attribue souvent la faute aux manœuvres des démagogues et truqueurs (voire à des services d'États étrangers), il a bien fallu que leur message rencontre un milieu favorable et soit repris par des médias adaptés, et il a fallu qu'il soit relayé. Soit dit en passant, par rapport aux propos qui se tenaient en 2011 le point de vue sur les interventions de médias étrangers ou sur la faculté des réseaux sociaux d'exprimer une opinion reflétant la sagesse des foules et qu'aucun gouvernement ne devrait pouvoir censurer ont singulièrement évolué en cinq ans.

L'idéologie dominante ne se reconnaît pas au fait que tout le monde y adhérerait (elle peut même être assez minoritaire), mais à sa position qui lui permet de réclamer le monopole de l'évidence ou des opinions acceptables sans scandale et souvent aussi à son programme d'en finir avec toutes les idéologies (puisqu'elle possède le vrai). Or c'est ce régime qui se détraque. 
Nos sociétés souffrent à la fois de surinformation (toutes les versions de la réalité en ligne), de clôture informationnelle (chacun peut s'isoler dans sa bulle de confirmation de la réalité) et de concurrence informationnelle (les deux camps s'accusant mutuellement de nier la réalité). Et la lutte entre les activistes et leurs adversaires devient moins un conflit pour dire ou révéler qu'une compétition pour l'attention et la confiance, deux ressources que le cerveau humain produit en quantité limitée et qu'il alloue de façon parfois surprenante. 



\section{Discussion}

Président (Jean Gicquel): Merci beaucoup cher collègue pour votre intervention, votre phrase «on ne vit plus dans le même réel», résume, je crois, parfaitement cette idée, ce décalage, c'est assez extraordinaire. Vous avez évoqué le complot de Vladimir Poutine lors de la campagne électorale américaine, mais Barack Obama avait déjà renouvelé la manière dont les élections étaient perçues sur les réseaux.

François-Bernard Huyghe: Il y a eu effectivement beaucoup de commentaires sur le fait que le clan démocrate de Barack Obama, utilisait, intelligemment, les réseaux pour délivrer son message et faisait un ciblage de clientèle absolument extraordinaire. C'est un autre élément. On voit ainsi le militant politique classique qui va dans telle maison en sachant qu'il doit là parler d'économie ou que l'électeur potentiel a un grand-père ukrainien, quelles sont ses préférences, etc. Le problème c'est que le «persuadeur», le rhétoricien, va savoir précisément quelles sont les attentes. Et encore, attendons d'être vraiment dans le big data. On dit déjà que Google en sait plus sur nous que nousmêmes. Les possibles rhétoriques que cela ouvre ne me font pas particulièrement rire.

Président (Jean Gicquel): Pour revenir à des aspects de droit constitutionnel: la rareté du recours au vote électronique. Il est dit que les procédures ne sont pas assez sécurisées, que les opérations de vote sont onéreuses... Il y a là aussi le facteur «temps», mais au dernier moment, on se dit «non», on ne peut pas informatiser cela, on ne peut pas violer ce principe essentiel: le vote est secret.

François-Bernard Huyghe: À mon avis, il est secret et solennel. Peut-être que l'on trouvera des solutions techniques pour garantir le secret du vote électronique pour ne pas se faire abuser. Mais solennel, cela me paraît important sur le plan symbolique, le citoyen vote en mettant son «beau costume du dimanche », cela fait partie du votant qui se sent comme une partie membre du souverain.

Public: Vous avez évoqué l'algorithme de Google qui permet de traiter les recherches, j'aimerais savoir comment cela marche. Est-ce que cela permet de détecter les auteurs, est-ce que cela permet de signaler?

François-Bernard Huyghe: Je ne suis pas un technicien, mais un pur littéraire. Vous avez des fonctions de repérage «à la chinoise», il faut faire une combinaison de touche pour taper des idéogrammes et quand vous commencez à taper un mot interdit (quand il manque un petit tiret pour que l'idéogramme veuille dire liberté ou autre): «bip!», votre écran vous annonce que vous allez écrire un mot interdit et que vous allez perdre des points. Ils ont un système de points. Quand je parle de l'algorithme de Google, c'est un moteur sémantique qui va analyser à partir des mots employés si l'on est en voie de radicalisation en cherchant des sites djihadistes, et Google vous envoie 
vers des vidéos vertueuses, du moins en théorie. Lors d'un colloque de l'UNESCO au Québec, histoire de faire le malin dans la session du lendemain, j'ai «joué » au djihadiste dans mon hôtel en utilisant internet pour chercher des contenus djihadistes. Mais la police ne m'a pas arrêté, je n'ai pas été repéré. Mais très honnêtement, pour le naïf, la probabilité de tomber sur des contenus djihadistes est extrêmement faible, elle est même nulle. Parce que vous allez tomber sur des pages et des pages entières, soit de définitions ou de considérations, soit sur des sites assez bien faits qui vont faire de la déconstruction («le djihad ce n'est pas l'islam»). Pour trouver désormais des discours djihadistes, il faut déjà être initié, finalement pour entrer dans un réseau virtuel, il faut déjà être dans un réseau humain, même s'il y a des moyens de contourner cela. Comment fais-je pour avoir des milliers de documents djihadistes? La réponse est simple, je vais sur des sites contre djihadistes.

Président (Jean Gicquel): Vous vivez dangereusement cher collègue!

François-Bernard Huyghe: Petit détail, j'ai quand même donné une de mes adresses IP à l'Unité centrale de lutte antiterroriste. J'ai donc un alibi!

Public (Pascal Jan): Sur le propos général que vous avez tenu notamment sur les dangers, l'absence de contrôle ou au contraire le contrôle de la pensée par des acteurs privés, c'est finalement de cela qu'il s'agit. J'aimerais réagir aux propos d'un collègue, Michel Bouvier, qui affirmait dans un édito, qui englobe vos propos: «tout semble indiquer que le passage à une société sans État fait d'ores et déjà parti des futurs possibles ». Qu'en pensez-vous?

François-Bernard Huyghe: C'est une des utopies qui a toujours animé internet et qui correspondait à la fin de l'autorité et au dépassement des territoires. Fin de l'autorité, je vois des pays qui contrôlent assez bien. Mais fin des territoires, ce n'est pas vrai du tout. On dit qu'internet a trois couches: une couche matérielle, une couche logicielle et une couche sémantique. La couche matérielle, ma tablette, je l'ai achetée quelque part, elle est liée à un WIFI quelque part, qui lui-même est lié à un fournisseur d'accès encadré par une certaine législation. La matérialité des choses vient se rappeler à nous en termes de pouvoir et de contrôle. Les logiciels sont produits quelque part. Si vous utilisez Windows ou si vous utilisez des logiciels nationaux en Chine, ce n'est pas la même chose. Quant à la dimension sémantique, il me semble qu'en dépit de tous ces discours d'un monde unifié, on a toujours une conception du monde différente selon les latitudes. Donc la fin de l'État tué par le numérique, j’attends de voir...

Public (Pascal Jan): L'idée était aussi l'existence de sociétés qui s'organisent indépendamment de toute autorité et sans véritablement de contrôle des autorités publiques.

François-Bernard Huyghe: Vous pouvez échapper à l'État. Vous pouvez aller, par exemple, sur le darknet, et faire des tas de choses illégales avec un risque quasiment nul d'être pris. Donc, si vous avez la ruse, la stratégie et la connaissance technique, on parle parfois de zone d'autonomie temporaire, «hacking bay», où l'on pouvait en quelque sorte vivre dans notre pays utopique et virtuel, hors du contrôle de l'État. Et il y a d'autres puissances qui se manifestent à travers le virtuel dont 
les GAFA [Google, Apple, Facebook, Amazon]; Google est pour moi l'une des premières puissances géopolitiques actuelles dont l'idéologie est le transhumanisme. Il faut le noter, il s'agit là de l'apparition d'autres acteurs, non étatiques: Google qui favorise les printemps arabes, Google qui menace la Chine, Google qui négocie directement dans le bureau de François Hollande, Google qui se moque de nos systèmes fiscaux européens, etc. Google me semble une puissance géopolitique évidente.

Public: Une question sur l'affaiblissement de l'esprit critique. L'université cherche à développer cet esprit pourtant certains étudiants posent des questions surprenantes du type, «Avez-vous vu la greffe de tête sur Paris Match?». Paris Match comme source pour un étudiant en thèse! Il y a un aplatissement de toutes les sources, un affaiblissement de l'esprit critique. Certains étudiants ne comprennent pas mon message.

François-Bernard Huyghe: Sur cette question de la critique, de nombreux projets ont été développés pour éduquer les jeunes à utiliser internet, à ne pas suivre toutes les rumeurs. À leur décharge, tout va très vite, il y a une surabondance, donc une mise à plat de toutes les sources. De fait, on aboutit à ce paradoxe: plus on a de sources plus s'informer coûte, soit financièrement soit en effort mental. Dans le domaine de la vérification, il est possible de «jouer collectif». Nous ne sommes pas les seuls «gentils »! Il y a beaucoup de gens qui passent leur temps à vérifier les sources, à faire du «fact checking », il existe même des logiciels de vérification des photos. Il est possible d'apprendre des choses très simples pour voir si une image que l'on dit de Syrie n'est pas finalement du Liban et n'a pas été prise cinq ans auparavant. Il y a de nombreuses techniques, si l'on fait l'effort de vérifier les sources que l'on peut avoir, si l'on fait confiance à des gens qui les vérifient avec nous, cela peut aller beaucoup plus vite. Mais nous revenons toujours au temps de cerveau humain. Il est facile, en plus, de répandre des rumeurs ou de se tromper. Moi-même, il m'est arrivé par hâte de mettre une photo du Hamas à la place du Hezbollah. Je me considérais de bonne foi et mon texte n'était pas invalidé par cela. Mais on se fait tous prendre. Pour ma part, personne ne m'a pris pour cela. (Rires)

Président (Jean Gicquel): Nous avons le sentiment, après avoir entendu les différents rapports, aussi brillants les uns que les autres, d'avoir enrichi notre pensée tout en étant inquiets de certaines perspectives. Cela montre encore la pertinence de l'objet de ce colloque. 



\section{PARTIE II}

GOUVERNANTS ET NUMÉRIQUE : ENTRE VÉRITABLE OPPORTUNITÉ ET RISQUE D'INSTRUMENTALISATION 



\title{
Propos introductifs
}

\author{
Pascal JAN ${ }^{1}$
}

Pour les gouvernants, la question numérique est telle une pièce de monnaie. Le côté face leur offre l'opportunité de rendre compte de leur action, de relayer leurs propositions, de diffuser de l'information institutionnelle... Sous cet angle, la distance entre gouvernants et gouvernés se réduit considérablement. Une sorte de lien de proximité s'installe, mais un lien purement virtuel et fragile. Le côté pile est la face cachée : par le biais des réseaux sociaux et des blogs personnels comme institutionnels, les gouvernants créent un environnement numérique auquel le citoyen est invité à s'inscrire, à participer se rendant rarement compte de l'enfermement intellectuel et politique dans lequel il s'engouffre, sauf pour lui à multiplier et diversifier ses sources et ses «connexions ». Par cette interaction entre les décideurs et les commettants, le numérique permet également aux gouvernants de «pister» les internautes, de connaitre leurs habitudes, leurs préférences et ainsi de cibler leur discours et de l'adapter à leurs interlocuteurs du moment. Car, en élargissant la réflexion aux formations politiques, la révolution numérique entraîne la mise en place de stratégies de conquêtes ciblées, personnalisées. Le risque d'une instrumentalisation est réel. Pour autant, ces manifestations nouvelles ne présentent pas que des aspects négatifs. Apporter des réponses précises et personnalisées aux citoyens répond à une demande légitime du corps électoral; à condition que ces démarches soient sincères dans leur objet et dans le contenu des informations délivrées.

Les communications qui vont suivre abordent la problématique sous l'angle des partis politiques et des élus. Sans empiéter sur le contenu de ces interventions, quelques observations à titre liminaire.

Il est certain que la révolution numérique bouleverse la représentation traditionnelle des intérêts des citoyens. Ceux-ci ne sont plus canalisés par les partis politiques. Ils s'expriment en dehors d'eux, spontanément, directement. Les formations et groupements politiques sont contraints de développer de nouvelles formes de militantisme, d'adapter leur discours aux exigences numériques (caractères limités des messages sur Twitter par exemple), d'opérer des campagnes de recrutement nouvelles dans leurs modalités... Par ailleurs, en Europe surtout, la révolution numérique facilite l'émergence

1 Professeur, Sciences Po Bordeaux. 
de nouveaux partis politiques qui rejettent l'idée même d'un endoctrinement au sein d'une structure donnée mais qui, très rapidement, reproduisent ce qu'ils dénoncent. Qu'il s'agisse de mouvements citoyens comme Podemos en Espagne ou le Mouvement Cinq Étoiles en Italie, la force du régime représentatif les oblige à s'organiser tôt ou tard en partis politiques. Quant aux mouvements d'une revendication thématique, si internet facile leur éclosion par une diffusion rapide et globale de leurs revendications, le temps des élections est pour eux souvent cruel en termes de transformation électorale. Le parti Pirate, essentiellement fondé sur la défense des droits à l'information, à la défense des données personnelles et confidentielles face à la puissance publique et à une transparence totale de l'action publique ne perce le temps que d'un éclair, d'une élection sans être en capacité de capitaliser les acquis électoraux sur le moyen terme. En fait, ces partis éphémères ne réussissent à pénétrer durablement les sphères institutionnelles que lorsque leur programme politique embrasse des problématiques générales, lorsqu'ils sont en capacité de conclure des alliances... et lorsque les États dans lesquels ils sont implantés traversent une crise politique, sociale ou économique sérieuse. Ce faisant, ils courent le risque de se diluer dans des ensembles plus vastes et englobants et de perdre leur spécificité et leur identité. Autre caractéristique de ces mouvements: ils contestent le système, les élites, la pensée dominante. Contestataires dans leur ADN, ces mouvements doivent leurs premiers succès à la révolution numérique mais ils sont incapables de fidéliser leur électorat numérique pour en faire un électorat politique permanent qui se manifeste concrètement dans les isoloirs. La révolution numérique profite à l'émergence et à la diffusion des idées contestatrices, créant un village où la mobilisation est fulgurante mais la démobilisation tout aussi rapide.

Les élus n'échappent pas à cette révolution numérique. Mais l'outil des réseaux sociaux et les blogs ouvrent surtout pour eux une opportunité d'exister sur la Toile. Ils participent à leur visibilité publique. De ce point de vue, et pour ne s'attacher qu'aux parlementaires nationaux, le numérique présente l'immense mérite pour les députés et les sénateurs de concrétiser la nature représentative de leur mandat. Ils s'adressent à l'ensemble des citoyens et non à leurs seuls électeurs. En période de campagne électorale, l'outil numérique revêt une autre fonction: tisser une toile dense de contacts, de réseaux partageant et relayant les idées et propositions des candidats mais dénigrant également les arguments des adversaires. Les campagnes électorales américaines sont un modèle du genre. L'élection présidentielle de 2016 qui a vu s'affronter une candidate du «système » et un candidat «anti système» a révélé une nouvelle dimension des réseaux sociaux: faire pièce, en atteignant directement l'électeur connecté, à un environnement médiatique très hostile. Ce faisant, Donald Trump et son équipe ont pu dénoncer et répondre aux éditos médias virulents tenus à leur encontre. Le droit de réponse est toujours possible et du coup alimente la presse traditionnelle qui s'en fait l'écho. Une façon comme une autre de pénétrer les médias classiques qui sont dans l'adversité par rapport à une candidature ou plus largement à une formation politique.

Il reste que l'exploitation des potentialités du numérique par les élus et les partis politiques n'en est qu'à ses débuts. La révolution numérique politique est en marche. Le citoyen en bénéficiera-t-il ou sera-t-il l'objet d'une instrumentalisation insidieuse au service d'intérêts personnels dont 
la finalité est l'accession aux responsabilités politiques? La vie politique et la communication des gouvernants et ceux qui prétendent le devenir peuvent-elles se contenter et dépendre de messages courts (tweet) et, parfois, simplistes?

Si la démocratie connectée réduit avantageusement la distance gouvernants-gouvernés, elle présente l'inconvénient de désacraliser cette relation entre décideurs et commettants, d'en simplifier exagérément le contenu au risque de déconnecter les citoyens des problématiques réelles et des solutions envisageables pour répondre aux défis d'un monde en pleine ébullition.

Nul doute que mes collègues MichaëlBardin («Les partis politiques face au numérique : entre adaptation stratégique et émergence de nouveaux mouvements»), Priscilla Jensel-Monge («L’utilisation des réseaux sociaux par les parlementaires : instrument de revitalisation de la démocratie représentative? ») et Idris Fassassi (Le rôle des réseaux sociaux dans la campagne électorale aux États-Unis) apporteront, par leur éclairage savant, des éléments d'explication et de réponse à cette question essentielle: Le numérique, véritable opportunité pour les gouvernants ou risque d'instrumentalisation de leur part? 



\title{
LES PARTIS POLITIQUES FACE AU NUMÉ RIQUE : ENTRE ADAPTATION STRATÉGIQUE ET ÉMERGENCE DE NOUVEAUX MOUVEMENTS
}

\author{
Michaël BARDIN ${ }^{1}$
}

$\mathrm{Au}$ moment d'appréhender l'influence du numérique ${ }^{2}$ sur les partis politiques, la tentation a été grande d'établir une classification basée sur l'impact réel du numérique dans le fonctionnement ou la constitution de mouvements politiques. Cela revenait à débuter par les partis dits «traditionnels », c'est-à-dire ancrés depuis longtemps dans la vie et la culture politique d'un État et à clore par ceux, souvent très récents, qui ont su profiter de l'internet et des technologies actuelles pour se faire connaître des citoyens.

Sans grande surprise, la communication est l'élément le plus commun aux différents partis. La communication numérique est le «sésame» des temps politiques modernes. L'internet et plus largement les technologies du numérique sont des instruments de communication indispensables. Naturelle, forcée, bien gérée ou non, aucun élu, aucun parti ne peut aujourd'hui faire l'économie d'une communication numérique. Et même s'il le souhaite, les réseaux sociaux sont désormais un relais incontournable et surtout non totalement maîtrisé par le candidat ou le parti. En revanche, la part du numérique dans la communication globale de ces partis reste très variable, sans qu'il s'agisse pour autant d'une question d'adaptation à l'outil.

Il est évident que pour un parti comme le Parti pirate, qui est «né sur internet » pour contester les premières mesures de lutte contre le téléchargement illégal, la communication numérique n'est pas un choix, mais un pis-aller pour au moins deux raisons. D'une part, le fondement même du parti est l'idée d'un partage absolu des données, quelles qu'elles soient; d'autre part, la communication numérique est également, aujourd'hui, la moins coûteuse des communications, la moins encadrée juridiquement, et sans doute celle qui sera le plus aisément relayée. Il en va de même du Mouvement 5 étoiles (Movimento 5 Stelle ou Cinque Stelle) en Italie, né du blog de Beppe Grillo et d'une structuration des échanges grâce aux réseaux sociaux.

\footnotetext{
1 Maître de conférences à l'Université d'Avignon, membre du LBNC (EA 3788), membre associé du CDPC Jean-Claude Escarras de l'Université de Toulon (UMR-CNRS 7318 DICE).

2 Aujourd'hui, il faut appréhender le terme «numérique » en tant que substantif. Il désigne ainsi l'ensemble des technologies actuelles de l'information et de la communication.
} 
Pour autant, les partis dits traditionnels ne sont en aucun cas fermés aux technologies actuelles, bien au contraire. Cela dit, il ne faut pas tomber dans l'illusion d'un «tout numérique » : ces partis sont dotés d'un ancrage électoral plus large, et ils doivent préserver leur lien avec les citoyens (souvent plus âgés) qui ne sont pas, ou peu, concernés par le numérique. Il n'empêche que tous les partis dits traditionnels ont fait de réels efforts pour franchir le «cap du numérique », d'autant plus d'ailleurs lorsqu'ils accèdent au pouvoir. Les exemples de cette utilisation nouvelle et parfois maladroite de l'outil numérique ne manquent pas. Ainsi, en Italie, Matteo Renzi a véritablement révolutionné la communication du gouvernement entre 2014 et 2016. Ce même gouvernement avait déjà démontré son intérêt pour l'utilisation du numérique avec la consultation publique sur les réformes constitutionnelles (Partecipa!) organisée par le Gouvernement Letta en 2013. Le gouvernement français a lui aussi fait montre d'une ouverture similaire à l'outil numérique avec une multiplication des consultations publiques.

Du point de vue strictement communicationnel, qu'il s'agisse d'assurer une visibilité au parti (ou à l'élu) ou bien de réinventer une participation citoyenne au processus de décision politique (et/ou juridique), les constats qui peuvent être faits aujourd'hui restent très mitigés.

Il convient, par exemple, de reconnaître que l'usage des outils numériques n'emporte pas une irrépressible adhésion qui se matérialiserait par une forte participation des citoyens lors des consultations. Ainsi, en France, il faut rappeler que la participation (en ligne) à la loi pour une République numérique s'est concrétisée par 147616 votes (provenant de 21409 participants) ${ }^{3}$, que la consultation sur le big data en santé a recueilli 326 «idées » ou encore que la participation à la consultation pour les réformes constitutionnelles organisée en Italie s'est soldée par 203061 questionnaires remplis4. Dès lors, même si ces consultations sont très appréciées sur le principe, le taux de participation des citoyens reste toujours très bas, et donc fort peu représentatif5.

Du point de vue de l'utilisation par les partis et les élus des outils numériques, là encore, et dans la lignée des propos de Priscilla Jensel-Monge ${ }^{6}$, l'utilisation de la communication numérique, si elle reste un véritable et nouvel atout, n'en est pas moins un instrument qu'il convient de manier avec prudence. La «faiblesse», si l'on peut la qualifier ainsi, de l'outil numérique est que «rien ne disparaît » et que «tout est exacerbé »: le tweet «malheureux» et immédiatement effacé sera malgré tout relayé, tout comme la photo jadis simplement diffusée dans une publication confidentielle sera visible dans le monde entier avec les réseaux sociaux. Les représentants politiques apprennent encore à gérer cet outil, souvent avec maladresse.

Autrefois réservé à quelques-uns, médiatiquement exposés, le phénomène de «surexposition de soi $»^{7}$ est devenu une réalité commune avec le développement des réseaux sociaux. Matteo Renzi en

Selon les chiffres du site créé à cette occasion, www.republique-numerique.fr.

Pour une synthèse de la consultation publique, «Partecipa!, l’Italie à l'heure de l'e-démocratie », La Lettre d'Italie, $\mathrm{n}^{\circ}$ 4, 2014, p. 34-35.

Pour rappel, l'Italie comme la France comptent plus de 45 millions d'électeurs.

Voir la contribution de P. JENSEL-MONGE, in cet ouvrage.

Voir notamment, F. Granjon, J. DenouËL, «Exposition de soi et reconnaissance de singularités subjectives sur les sites de réseaux sociaux », Sociologie, 2010/1, p. 25-43; L. Mell, «Hors de l'ombre. Analyse diachronique du phénomène de mise en visibilité numérique de soi », 
a sans doute fait l'amère expérience: son omniprésence dans les médias et sur les réseaux sociaux, pour relayer l'action de son gouvernement, a fini par «lasser» l'opinion publique. Dès 2014, un éditorial du directeur du Corriere della Sera, Ferruccio de Bortoli, identifiait «l'ennemi dans le miroir » (Il nemico allo specchio) et affirmait qu'en cherchant à mettre en avant chaque action gouvernementale, le président du Conseil en exposait surtout les faiblesses et laissait à penser qu'il cherchait surtout à promouvoir sa propre personne. Certes, cette maladresse n'est pas en elle-même responsable de l'échec politique de Matteo Renzi, mais, et contrairement à l'effet recherché, sa surexposition a sans doute fait passer au second plan son action proprement dite. Cela dit, cette difficulté est aujourd'hui le lot de tous les responsables politiques. En France, la prise de pouvoir (et certaines prises de position) de Marlène Schiappa en tant que secrétaire d'État s'est avérée parfois chaotique: entre des tweets parfois postés «à titre personnel» et d'autres en tant que «membre du gouvernement » (mais tous postés depuis le même compte Twitter) ou la publication de photos prises de nuit dans un quartier de Paris en pleine polémique sur le harcèlement de rue notamment dans ce même quartier. Cette publication a finalement été effacée de son compte et présentée par son équipe comme un simple «bug communicationnel».

L'exemple est aussi parlant que redondant. Une redondance laisse d'ailleurs penser que cette communication numérique (notamment via les réseaux sociaux) tient plus des monologues «reliés »ou «superposés » que de véritables échanges. Cette communication permet tout autant aux politiques de «réaffirmer des vérités » - sans aucun contradicteur direct - sur des évènements passés (une participation à une émission télévisée ${ }^{8}$ ) que de «réinterpréter» des propos tenus parfois quelques heures plus tôt ${ }^{9}$.

La démocratie connectée, illustrée par une communication numérique exponentielle, ne présente donc pas que des avantages pour les partis politiques et leurs représentants. Elle met ainsi en œuvre un mode de communication dont l'étendue leur échappe souvent et qui est, trop régulièrement, un moyen d'éviter un véritable échange. Fort heureusement, le numérique ne saurait être réduit à de la communication pure et simple. Il est également à l'origine d'évolutions qui sont d'une tout autre ampleur pour la vie politique actuelle.

Le numérique est politique, il s'agit dorénavant une certitude. Les technologies actuelles font le lit - de manière inédite - d'une «autre politique» ou tout au moins de nouveaux mouvements

Questions de communication, $\mathrm{n}^{\circ} 28,2015$, p. 231-250

8 À titre d'exemple, Jean-Luc Mélenchon, au lendemain de sa participation à «L'émission politique» sur France 2 en décembre 2017, publiait sur son blog un long texte où il affirmait: «C'est l'unique moyen dont nous disposons pour achever de transformer en une déroute morale dans le grand public ce traquenard une nouvelle fois organisé contre moi par France 2 » ou encore que «ce que nous avons vu ce soirlà, ce n'est plus du journalisme, ce n'est plus une émission du service public, c'est juste un règlement de compte entre une équipe de gens prête à toutes les mystifications pour frapper le principal opposant politique à ses employeurs. Une manœuvre politicienne sous déguisement journalistique», 4 décembre 2017, https://melenchon.fr.

9 Dans le même esprit, alors qu'une sénatrice se plaignait, en séance, de l'usage de la cigarette dans le cinéma français en estimant qu'il participait «peu ou prou à banaliser l'usage, si ce n'est à (...) promouvoir [le tabac], auprès des enfants et des adolescents », Agnès Buzyn, la ministre de la Santé répondait: « Je rejoins totalement ce qu'a dit Nadine Grelet-Certenais sur le cinéma français. Je veux qu'on ait une action ferme là-dessus. Je ne comprends pas l'importance de la cigarette dans le cinéma français ». Devant la polémique que soulevaient de tels propos, notamment dans le monde du cinéma quant à la liberté d'expression dans la création, la ministre, sur twitter, précisait: «la liberté de création doit être garantie. La sénatrice à laquelle je répondais jeudi ne le proposait pas non plus. Cette polémique n'a donc pas lieu d'être ». Si la ministre n'a effectivement pas proposé d'interdiction, elle n'a pas non plus expliqué ce qu'elle entendait par «une action ferme » avant de «clore» la polémique. 
qui se fondent sur cette idée (I). Derrière cette affirmation de départ se dissimulent des réalités très disparates d'un point de vue démocratique (II). Cela dit, le numérique exerce une influence omniprésente, sans aucun discernement quant aux partis, dès lors qu'il est question de campagnes électorales (III).

\section{L'émergence de nouveaux mouvements}

Le numérique réduit les distances: entre la conviction et l'engagement politique ou encore entre le pouvoir et le citoyen. Dans tous les cas, il permet de cristalliser un sentiment de rejet des partis politiques, quitte à aboutir à en créer d'autres (A) en faisant du citoyen, et de sa participation, le symbole d'un renouveau (B).

\section{A. L'antipartisme organisé... en parti politique}

Le numérique est un formidable «accélérateur de démocratie», mais il change également, dans une profondeur qui reste à déterminer, les démocraties. En ce sens, l'illustration la plus flagrante de cette évolution est l'émergence de nouveaux mouvements.

Tout s'écrit et se dit depuis quelques années sur ces nouveaux mouvements, qui sont souvent définis comme les premiers éléments d'une e-démocratie, c'est-à-dire comme ceux qui incarnent la politique du futur, le basculement d'une démocratie de représentation à une démocratie de participation, la «politique $2.0 »$ en somme.

Bien qu'il existe différents «modèles », il est possible de s'accorder sur quelques éléments communs à toutes ces formations.

La naissance de ces mouvements est guidée par un phénomène de rejet des partis et du système existants qui fait son lit d'un contexte économique, politique et social difficile. La naissance et le succès de Podemos en Espagne ou du Mouvement 5 étoiles en Italie sont directement reliés aux difficultés des deux États.

Dans le cas de l'Italie, les dysfonctionnements récurrents du régime parlementaire ou encore les errements d'une partie de la classe politique ont fait d'un parti créé en 2009, le premier parti du pays dès 2013 (puisqu'il n'est devancé que par deux coalitions) avec quelque cent neuf députés et cinquante-quatre sénateurs. Même si, a posteriori, la mesure était indispensable, la mise en place d'un gouvernement dit «technique» (entre 2011 et 2013), basé sur la mise en œuvre nécessaire de mesures d'austérité a favorisé l'émergence d'un parti conduit par un comique spécialiste de la caricature politique, Beppe Grillo. Mais, au-delà du mouvement d'humeur, et dans un contexte politique particulier (à savoir un centre-droit désorganisé et un centre-gauche dominé par le Parti démocrate et la main de fer du président du Conseil des ministres Matteo Renzi), lors des élections européennes de 2014, le parti recueille encore plus de $20 \%$ de votes arrivant juste derrière le Parti démocrate. En juin 2016, les Grillini remportaient les élections municipales à Rome et à Turin. Le cas de Podemos 
en Espagne, fondé en 2014, s'explique lui aussi par une crise économique et sociale qui dure depuis 2008 et par un bipartisme quasiment inchangé depuis la transition démocratique. Dans les deux cas, la nécessité de changement s'est imposée en l'absence de réponse des représentants. Pour autant, la naissance de ces mouvements n'est pas toujours guidée par un malaise aussi «profond» comme le démontre l'exemple du Parti pirate.

Pour rappel ${ }^{10}$, le Parti pirate (Piratpartiet) est né en Suède, en 2006, à l'initiative de Rickrard Falkvinge, un ingénieur et ancien chef de projet chez Microsoft. Le choix même du nom du parti apparaît comme une réponse, en forme de bravade, aux premières mesures de lutte contre le téléchargement illégal. Ainsi, la Suède est l'un des premiers pays à se doter d'un «Bureau antipiratage » (Antipiratbyrån) sous l'impulsion de l'industrie musicale. En réaction, les partisans suédois du libre partage des fichiers fondent, en 2003, le «Bureau du piratage » (Piratbyrån). Dans les mois suivants, certains membres de ce Bureau créent un site d'échange de fichiers, The Pirate Bay, qui deviendra l'un des plus populaires et des plus connus au monde, tant pour son utilisation que pour ses démêlés judiciaires. Le mouvement devenu très populaire se concrétise finalement, en 2006, par la fondation à Stockholm du Piratpartiet.

À dire vrai, ce ne sont pas tant les résultats électoraux du Parti pirate qui sont remarquables que l'établissement et la diffusion d'un modèle par le seul biais d'internet. En effet, au plus haut de sa popularité, le parti suédois recueillait un peu plus de $7 \%$ des votes lors des élections européennes de 2009 ce qui ne permettra qu'à deux élus pirates de siéger au Parlement. De même, la création d'un Parti pirate français n'a pas vraiment bouleversé la vie politique de l'Hexagone: une centaine de candidats présentés lors des élections législatives de 2012 qui rassemblent un peu plus de 32000 voix (et donc aucun élu), un seul conseiller municipal élu en 2014. Lors des élections régionales de 2015, le Parti pirate n'est présent qu'en soutien de candidats d'autres mouvements. Enfin, lors des élections législatives de 2017, si le parti investit cinquante-huit candidats, le plus haut score obtenu par l'un d'entre eux ne sera que de 1,42\%. À l'inverse, la création du Parti des pirates en Allemagne (Piratenpartei Deutschland) emportera immédiatement un insolent succès comptant jusqu'à cent soixante conseillers municipaux et quelque quarante-cinq parlementaires régionaux. Un succès qui est pourtant rapidement retombé puisque les élections régionales de 2016 et 2017 ont conduit à la perte de tous les sièges précédemment obtenus.

En bref, avec des scores électoraux relativement anecdotiques, le Parti pirate aurait pu, et même aurait dû, tomber dans une forme d'oubli si de récentes élections en Islande (en 2016) et en République tchèque (en 2017), n’avaient pas donné un nouvel élan au parti.

Déjà représentés par trois députés au sein de l'Althing (le parlement islandais), à l'occasion des élections législatives de 2016, les pirates islandais étaient crédités par les sondages de près de $20 \%$ des intentions de vote et, par le jeu des alliances, pouvaient espérer gouverner. Même si le résultat

10 Pour une étude consacrée au Parti pirate, M. BARDIN, «Quand internet rencontre la politique : l'exemple du Parti pirate », RLDI, $\mathrm{n}^{\circ} 107$, 2014, p. 84-91. 
est finalement en deçà des espérances (avec 14,5\% des votes et dix députés sur les soixante-trois sièges que compte l'Althing), la progression reste remarquable. Dans le même sens, l'élection d'un sénateur pirate en 2012 pouvait paraître de moindre importance, tant le candidat était préalablement connu et soutenu par une large coalition ${ }^{11}$, pourtant le succès du Parti pirate tchèque (Česká pirátská strana) n'a eu de cesse de se confirmer. À l'occasion des élections municipales de 2015, le parti siège au conseil municipal de Prague (quatre sièges) et dispose d'une dizaine d'élus dans différents conseils municipaux du pays et obtient même l'élection d'un maire pirate (commune de Marienbad). Enfin, après les élections législatives de 2017, le Parti pirate tchèque fait son entrée à la Chambre des députés (en obtenant 10,79\% des voix, soit vingt-deux députés) et devient le troisième parti politique du pays.

Malgré cela, et au-delà de ces rares exceptions, la force du Parti pirate n'est pas son nombre d'élus ou sa capacité à influer sur la vie politique d'un État, mais bien la diffusion de son modèle, à l'échelle internationale de surcroît. En effet, plus encore que la naissance même de ce qui n'était jamais, au départ, qu'un «petit» parti suédois, c'est le retentissement mondial de cette initiative qui est intéressant. Ainsi, la première force de ce mouvement est sans doute sa capacité à «s'autopromouvoir ». Bien avant que les médias ne s'emparent du phénomène (et ne le popularisent plus encore), le Parti pirate suédois va inspirer la création de partis pirate dans toutes les régions du monde, jusqu'à être aujourd'hui présents dans plus de soixante pays. La multiplication du modèle est la démonstration de la «force»d'internet (qu'elle soit positive ou non d'ailleurs), avec peu de soutien médiatique et des financements souvent très limités, les partis pirate ont pourtant réussi à se multiplier.

L'organisation de cette «piraterie» aurait pu en rester à de simples initiatives nationales ou locales, mais un pas vers une «fédération de partis» est franchi le 18 avril 2010 avec la création d'un Parti pirate international. Reposant sur le statut d'association belge à but non lucratif, ce parti adopte de véritables statuts avec à sa tête un comité directeur et deux coprésidents. L'objectif est simple : tenter d'établir un lien entre les partis pirate du monde entier afin de dégager des orientations politiques communes ou encore de promouvoir la création de nouveaux partis pirate ${ }^{12}$. Là encore, ce qui aurait pu apparaître comme anecdotique a été exploité très rapidement pour participer aux conférences internationales. Ainsi, après avoir essuyé un refus de l'OMPI (Organisation mondiale de la propriété intellectuelle), le parti obtient en revanche le statut d'observateur auprès de l'OMC (Organisation mondiale du commerce) pour la conférence ministérielle organisée à Bali en décembre 2013. En accédant à cette conférence, c'est un véritable statut international qui est octroyé au mouvement qui a pu envoyer quatre représentants pour défendre ses points de vue lors des négociations (négociations qui sont fermées au public). En effet, comme le prévoit l'annexe 3 du règlement intérieur des

11 Libor Michálek s'est fait connaître en République tchèque comme le lanceur d'alerte qui a dénoncé un important scandale de corruption au sein du ministère de l'Environnement. Il a été élu sous l'étiquette du Parti pirate, mais il était également soutenu, y compris durant sa campagne, par les Verts et les Chrétiens-Démocrates.

12 L'adhésion des formations nationales du Parti pirate n'a rien d'obligatoire et, en ce sens, il existe ainsi différents types de membres au sein du Parti pirate international. À l'image de nombreuses organisations, à commencer par exemple par la pratique instaurée au sein de l'Organisation des Nations unies depuis 1946, le Parti pirate international compte des membres «ordinaires » et des membres «observateurs ». Selon les statuts du Parti pirate international, les membres ordinaires doivent adhérer « aux statuts et aux buts de l'association» alors qu'aucune obligation ne pèse sur les membres observateurs. 
conférences ministérielles de l'OMC ${ }^{13}$, le but du statut d'observateur auprès de l'OMC est de permettre à ces organisations «de suivre les discussions portant sur des questions qui les intéressent directement $»$ et de «prendre la parole lors des réunions $»^{14}$.

Un second pas est franchi en 2014, avec la création, à l'occasion des élections, d'un Parti pirate européen, ayant vocation à uniformiser la campagne européenne des partis pirate nationaux et ensuite à coordonner, au sein du Parlement, les travaux de ses élus.

La première force du Parti pirate est donc sa capacité d'organisation et de multiplication. La souplesse structurelle et la possibilité d'utiliser librement l'étiquette «Parti pirate» (la moindre des choses pour un mouvement luttant contre les brevets ou encore les monopoles...) ont permis à cette organisation d'être très rapidement représentée dans de nombreuses élections, locales, nationales et même européennes. Mais en définitive, le Parti pirate est une sorte de « coquille vide » puisqu'au-delà de la dénomination, le point commun est la mise en œuvre d'un libre accès « aux informations culturelles et scientifiques» (selon les termes des statuts du Parti pirate international). Plus clairement, le Parti pirate se propose comme une organisation, peu marquée politiquement, qui permet aux candidats potentiels comme aux électeurs d'exprimer leur défiance à l'égard des partis traditionnels.

C'est plus clairement encore cette défiance à l'égard de ces partis et même un antipartisme assumé qui a guidé la naissance d'un second modèle en Espagne et en Italie.

\section{B. Le citoyen comme garant du renouveau de la classe politique}

L'avènement d'internet a donné corps à une contestation latente à l'égard des partis et même de la classe politique. Fondée ou non, cette contestation n'a eu de cesse de s'amplifier avec les difficultés économiques rencontrées, entre autres, par les États européens. Au gré des difficultés, le souhait de renouveler une classe politique qui est toujours - à tort ou à raison - perçue comme une «caste de privilégiés » s'est mué en une véritable volonté de replacer le citoyen au cœur du débat politique.

Cette méfiance à l'égard des partis est présente à divers degrés dans toutes les formations nées sur internet. Si, à l'inverse du Parti pirate, internet et la communication numérique ne sont pas toujours les instigateurs des mouvements, ils en sont systématiquement les catalyseurs.

Ainsi, Podemos trouve son origine dans le Mouvement des indignés (dont la véritable dénomination est «mouvement $15-\mathrm{M} »$ pour 15 mai 2011 date des manifestations organisées dans plus de soixante villes espagnoles et dans quelque cinquante autres villes dans le monde). Cette initiative du mouvement 15-M va être immédiatement relayée par une association citoyenne espagnole «La vraie démocratie maintenant» (dont le site internet porte, en sous-titre, la mention «Nous ne sommes pas des marchandises dans les mains des politiciens et des banquiers »), disposant d'une large audience

13 Conférences de l'OMC, Règlement intérieur, www.wto.org/french/tratop_f/inftec f/git3.doc.

14 Toujours selon le même article, «le droit de prendre la parole ne comprend pas le droit de distribuer des documents ou de faire des propositions, à moins qu'une organisation ne soit invitée expressément à le faire, ni à participer à la prise de décisions ». 
sur internet et sur les réseaux sociaux. Ce mouvement est rapidement soutenu par un collectif d'universitaires - inquiets de la précarité affectant la jeunesse - qui va créer en février 2011 un mouvement appelé «Jeunesse sans futur». Si les manifestations vont se multiplier entre 2011 et 2014, le point d'orgue de ce mouvement citoyen est la Marche pour la Dignité qui s'est déroulée à Madrid en mars 2014, soit quelques semaines avant les élections européennes.

Cette importante mobilisation fait écho à la publication en janvier de la même année d'un manifeste (signé par une trentaine d'intellectuels, de journalistes et autres personnalités de la société civile) intitulé «Prendre les choses en main: convertir l'indignation en changement politique » dans le journal numérique espagnol Público. Quelques jours plus tard, le parti politique Podemos était né. Pour sa première participation à des élections, la nouvelle formation se hisse à la quatrième position sur l'échiquier politique du pays, avec $8 \%$ des voix et cinq sièges. Ce succès, inattendu, est essentiellement basé sur un programme reprenant les principales revendications des manifestants anti-austérité (lutte contre la corruption, défense des services publics, audit public de la dette...) et une tête de liste médiatique, Pablo Iglesias, un jeune professeur de science politique, très présent dans les débats télévisés et actif sur les réseaux sociaux.

Cette montée en puissance se confirme lors des élections législatives de 2015 puisque Podemos fait élire soixante-neuf députés et seize sénateurs, un score maintenu lors des élections de $2016^{15}$.

Ce succès électoral n'atténue pas pour autant le caractère «antiparti » de la formation. Podemos «n'est pas un parti politique, il n'est enregistré comme tel que pour des raisons légales», comme n'hésite pas à l'affirmer son leader, chaque fois qu'il le peut.

Le politologue espagnol José Ramón Montero Gibert donne une définition de Podemos qui est assez caractéristique de ces nouveaux mouvements. Il estime ainsi qu'il s'agit d'une « «force politique [dont les] caractéristiques idéologiques initiales combinent, à des doses plus ou moins importantes, et de manière extrêmement simplifiée, le néopopulisme, l'anti-européanisme, l'antipartisme, et l'anti-systémisme, avec un gauchisme maximaliste appliqué sans beaucoup de nuances à toutes les causes et à tous les dirigeants ${ }^{16}$.

De ce point de vue, le positionnement de Podemos au sein de la classe politique espagnole rappelle celui du Mouvement 5 étoiles italien.

Beppe Grillo a toujours défini son mouvement comme un «non-parti ». Ce dernier dispose bien d'un statut, comme l'y oblige la loi italienne, qui est ainsi dénommé «non-statut $»^{17}$ et qui fait état d'une «non-association» (art. 2 du non-statut). Pour autant, et contre toute attente, les velléités politiques de Beppe Grillo et des Grillini ont toujours été au cœur du mouvement et le statut ne le nie d'ailleurs pas en affirmant à l'article 4 que le «Mouvement 5 étoiles n'est pas un parti politique

\footnotetext{
15 Avec la coalition Unidos Podemos et d'autres accords scellés après les élections, le groupe parlementaire Unidos Podemos - En Comú Podem - En Marea, compte aujourd'hui 67 députés et 20 sénateurs.

16 J. R. Montero, «Podemos como laboratorio político », El País, 13 juin 2014.

17 Disponible sur le site du Mouvement 5 étoiles, www.movimento5stelle.it.
} 
ce qui ne veut pas dire qu'il n'en deviendra pas un dans le futur». Une affirmation qui prend tout son sens au regard du contexte qui a accompagné l'émergence du mouvement: Les Amis de Beppe Grillo n'est jamais que la coordination, à partir de 2005, par le comique lui-même, via le réseau social Meetup, des «déçus» de la politique. L'idée que des listes civiques participent à des élections politiques, qui se concrétise en 2007, est non seulement antérieure à la fondation du parti, mais elle en est la justification. L'idée de non-parti est donc un positionnement anti-establishment que résumait parfaitement Carla Ruocco en 2013 juste après son élection à la Chambre des députés: «Nous serons tout ce que vous n'avez pas vu jusqu'à présent». Un positionnement qui est, il faut le reconnaître, clairement assumé par le parti qui a toujours refusé, par exemple, de bénéficier des financements publics offerts par la loi italienne aux partis politiques ${ }^{18}$.

Cette volonté de rupture avec la classe politique est le fondement même du mouvement mais aussi de son activité politique puisqu'en pratique, et à l'inverse de Podemos qui s'affiche comme une force politique d'appoint (c'est-à-dire prête à éventuellement s'allier avec les partis traditionnels), le Mouvement 5 étoiles rejette toute alliance d'envergure avec les partis traditionnels.

Ces partis, quasiment nés sur internet, prônent une cyberdémocratie. Une notion qui suggère une double idée: celle d'une influence directe du citoyen sur le représentant, et celle, plus difficile à cerner que le représentant doit justement être plus représentatif des citoyens. En bref, la mise en œuvre d'une politique permettant, selon le porte-parole de Podemos, aux «gens normaux de faire de la politique».

Cette défiance à l'encontre de «l'establisment partisan» trouve très clairement des applications pratiques, au moins sur la question du renouveau de la classe politique.

Ce renouveau est institutionnalisé par les statuts du Mouvement 5 étoiles puisque les règles permettant de se porter candidats lors d'élections locales ou nationales sont draconiennes (et ce sont les mêmes que pour adhérer au mouvement): ne pas avoir déjà participé, sous une autre étiquette, à une compétition électorale, ne pas être déjà membre d'un parti ou d'une autre association «ayant un objet ou un but $\gg^{19}$ similaire ou contraire à ceux du mouvement (soit un but politique), avoir un casier judiciaire qui est vierge (ce qui interdit d'ailleurs à Beppe Grillo lui-même de se porter candidat puisqu'il a fait l'objet d'une condamnation en 1980). Autant dire qu'avec de tels prérequis, le Mouvement 5 étoiles s'évite tout problème de transfugisme... Si Podemos est moins précis sur la question, il faut bien reconnaître que le système de primaires très ouvertes et en ligne permet une sélection qui conduit à la désignation de candidats issus de la société civile et la plupart du temps avec une expérience plus militante que politique.

Dans les deux cas, comme l'on peut s'y attendre, ces mouvements laissent une large place à des profils habituellement sous-représentés au sein de la classe politique : des élus qui sont donc souvent

18 À titre plus anecdotique, les élus du mouvement refusent la dénomination d'onorevole (littéralement «honorable») qui désigne traditionnellement les membres du Parlement italien, et se définissent comme de simples cittadini (citoyens).

19 Art. $5 \mathrm{du}$ non-statut. 
jeunes (une moyenne d'âge d'environ trente-cinq ans), une parité très largement respectée et, c'est un aspect intéressant de ces partis (qui démontrent bien qu'ils se construisent sur un rejet des partis traditionnels), qu'il s'agisse des adhérents ou des élus, leurs profils dépassent souvent les clivages socioprofessionnels classiques.

L'antipartisme de ces formations et de ses élus s'exprime également dans le rapport qu'ils entretiennent avec les charges électives auxquelles ils accèdent, notamment concernant les indemnités auxquelles ils peuvent prétendre en tant qu'élus. Ainsi, tous les candidats du Mouvement 5 étoiles s'engagent à rembourser à l'État leurs indemnités au-delà de 5000 euros mensuels, de même que la première décision des cinq eurodéputés de Podemos aura été de refuser de toucher les 8000 euros qu'attribue le Parlement européen à chaque parlementaire. Dans le même sens, les élus au Congrès espagnol, en adéquation avec le règlement interne du parti, s'engagent à renoncer à certains avantages que leur confère leur mandat: limitation des salaires (les élus de Podemos s'engagent à ne percevoir aucune indemnité supérieure à trois fois le salaire minimum qui est de 648 euros en Espagne), d'une partie des indemnités de logement ou encore refus de se faire payer leurs déplacements. Le parti entend ainsi «montrer l'exemple» et mettre en avant un rejet des «privilèges ». Les sommes auxquelles les parlementaires renoncent sont allouées, soit au frais de fonctionnement du Congrès des députés, soit mises à la disposition de projets sociaux.

En définitive, ces partis tentent bien d'insuffler de nouvelles pratiques dans la gestion des charges électives. Cela dit, une analyse plus globale de leur fonctionnement laisse plus perplexe.

\section{Les nouveaux partis et la difficile conciliation entre transparence et efficacité}

Le point commun à ces nouvelles formations politiques est l'idée d'une démocratie renouvelée et participative par le biais d'internet et des technologies offertes par le numérique. Mais au-delà de l'idée initiale, il convient de mettre en œuvre des modalités de fonctionnement en adéquation avec cette philosophie. De ce point de vue, les risques sont légion pour ces nouveaux mouvements : tenter de ne pas reproduire les schémas existants (A) tout en conservant une influence réelle sur la vie politique (B).

\section{A. Les risques d'un « centralisme cybercratique»}

L'idée d'une cyberdémocratie, pour reprendre un terme à la mode, mais aux contours quelque peu abscons, renvoie à celle d'une «démocratie liquide » ou «délégative » qui offrirait une synthèse de la démocratie représentative et de la démocratie directe. 
Il ne suffit pas de l'affirmer, faut-il encore la mettre en œuvre, et c'est sans doute là que le bât blesse. De ce point de vue, seul le Parti pirate met en œuvre un tel mode de fonctionnement notamment par l'utilisation du logiciel Liquidfeedback, sorte de plateforme d'idées qui peut être enrichie par les utilisateurs et qui permet la discussion, le vote et l'adoption de propositions.

En y regardant de plus près, des partis comme Podemos et a fortiori le Mouvement 5 étoiles sont assez loin de telles règles. Ils fonctionnement assez largement comme des partis dits traditionnels avec, dans les deux cas, ce que certains désignent comme un «centralisme cybercratique ${ }^{20}$.

Ainsi, les statuts de Podemos (consultables en ligne ${ }^{21}$ ) affirment que le parti est «l'expression politique d'un processus social massif d'autonomisation populaire $»^{22}$. Il est fondé autour d'une «Assemblée citoyenne » regroupant l'ensemble des adhérents, d'un «Conseil citoyen » constitué de dix-sept dirigeants locaux et nationaux, soixante-deux membres élus par l'Assemblée citoyenne et d'un Secrétariat général. Par ailleurs, et là les statuts du parti espagnol sont plus discrets, un «Conseil de coordination» de dix à quinze personnes est nommé par le Conseil citoyen, sur proposition du secrétaire général. Ce dernier conseil a pour fonction de coordonner les travaux des différentes instances et d'assumer celles qui ne relèveraient pas du Conseil citoyen.

Face à une telle structure, certains observateurs n'ont pas hésité à faire un parallèle entre cette organisation et celle du parti communiste soviétique ${ }^{23}$. La souveraineté est bien confiée à la base, mais les décisions politiques sont prises par le sommet. En pratique, l'examen minutieux des statuts permet d'affirmer que l'influence de la base ne s'exerce de manière effective qu'au moment de la désignation des organes de direction et surtout du secrétaire général. En dehors de cette période, toutes les instances sont subordonnées aux décisions politiques de ce dernier. La seule opposition possible, prévue par les statuts, est une procédure de destitution qui ne peut être mise en œuvre qu'à la demande des deux tiers des membres de l'Assemblée citoyenne, donc des deux tiers de la totalité des adhérents au parti ${ }^{24}$.

Pour le Mouvement 5 étoiles, le «non-parti » repose donc sur un «non-statut» qui se résume à huit articles. En dehors du fait d'affirmer que le mouvement est « une plateforme de discussion et de consultation qui a son origine et son épicentre sur le site internet du mouvement $»^{25}$, que l'adhésion est gratuite et que la participation se fait exclusivement par l'intermédiaire du site internet, il n'existe pas de règles écrites sur le fonctionnement ou les modalités de participation des adhérents. Et pour cause, cette participation se concrétise par des sondages et des questions qui sont déterminés sur le fond comme sur la forme par les dirigeants du parti.

\footnotetext{
20 Entre autres, S. CECCANTI, S. CURRERI, «I partiti antisistema nell'esperienza Italiana: il MoVimento 5 Stelle come partito personale autoescluso », Diritto pubblico comparato ed europeo, ${ }^{\circ}$ 3, 2015, p. 799-832.

21 Disponibles sur le site de Podemos, l'organisation, la ligne politique et idéologique du parti sont présentées dans quatre livrets (Documentos de Podemos): Documento organizativo, Documento político, Documento ético, Documento de feminismos, https://podemos.info.

22 Documento organizativo, «Préambule».

23 A. ElorzA, «Podemos ou le léninisme aimable», Histoire \& Liberté, n 58,2015 , p. 115-128.

24 Documento organizativo, art. 26.

25 Art. $^{\text {er }}$ (nature et fondement) du non-statut.
} 
Plus encore, ce non-statut a fait l'objet d'une révision en septembre 2016, et s'est vu complété par un règlement interne, dont le but principal et affirmé est d'établir un code de déontologie pour les élus du mouvement (accompagné de sanctions en cas de non-respect), mais aussi, et cela s'est fait avec beaucoup plus de discrétion, de définir les institutions qui guident le parti. Ainsi, le règlement dans son article 1 bis, prévoit que les organes du parti sont toujours «l'assemblée des membres » (les adhérents au site) et le leader politique (Beppe Grillo) désormais complétés par deux nouveaux organes «sans fonction directive ou représentative » : un «Collège d'arbitres » et un «Comité d'appel».

Ce collège est une «demi-nouveauté»: composé de trois membres, ces derniers (des parlementaires du parti) seront désignés par les adhérents (via un vote sur internet), mais sur «sur proposition du leader politique du mouvement». Le collège restera en fonction pendant trois ans et ne pourra être renouvelé dans cette fonction. Il interviendra pour juger des violations des règles déontologiques et décidera en conséquence des sanctions prises (expulsions, suspensions...). Cette «évolution» démontre, une fois de plus, qui est le seul et unique leader du mouvement.

Cela dit, cette forte personnalisation du mouvement autour du personnage de Beppe Grillo n'est plus aujourd'hui un choix. La pratique l'a démontré. Après avoir été omniprésent entre 2009 et 2014, un «directoire» composé de six parlementaires est mis en place. L'expérience s'est avérée si peu probante que même si ce directoire existe toujours, le comique italien a dû revenir sur le devant de la scène politique à peine quelques mois plus tard afin de mettre un terme aux dysfonctionnements internes du parti ${ }^{26}$. Une situation qui confirme les propos du politologue Paolo Pombeni qui affirmait en $2013^{27}$ que Beppe Grillo n'était pas un dirigeant politique, mais un «messie » et que «la forme d'organisation du consentement par Grillo est la même que pour le fascisme, le nazisme, le stalinisme et les grands régimes totalitaires. Tout est basé sur le fait que le leader est infaillible, quand on admet qu'il s'est peut-être trompé, tout l'édifice s'effondre $»^{28}$. Une chose est sûre, à l'heure d'une «mode des primaires », l'implication directe du citoyen dans le processus de décision politique, prônée par ces partis reste assez limitée.

Par ailleurs, la concrétisation exclusive ou presque de la participation des adhérents par le biais d'internet présente au moins deux limites qui mettent à mal le caractère démocratique de ces mouvements : un monopole sur les décisions à prendre et un consensus limité quant aux décisions prises.

En premier lieu, qu'il s'agisse de Podemos ou du Mouvement 5 étoiles, les partis détiennent le «monopole de l'information». En effet, et contrairement au Parti pirate, les adhérents ne sont que peu partie prenante aux orientations politiques du parti. En effet, ils ne peuvent se prononcer que sur

\footnotetext{
26 Ces dysfonctionnements ont conduit à la démission du parti de trente-six parlementaires durant l'année 2014.

27 Dans un entretien accordé au journaliste P. Vernizzi, «Pombeni : Beppe Grillo come Stalin, chi lo critica è pazzo e merita il manicomio », 12 juin 2013, www.ilsussidiario.net.

28 Paolo Pombeni précisait: «Évidemment, je ne veux pas dire que l'idéologie de Grillo serait semblable à celle du nazisme, et encore moins mettre en relation les deux phénomènes pour dire que les grillini seraient des nazis. Le mécanisme de fonctionnement du M5S est similaire à celui du fascisme, du nazisme et de tous les mouvements messianiques. Le problème concerne la façon d'organiser le consensus, qui est basé sur un guide qui peut alors être appelé Führer, Duce ou de toute autre manière, mais qui par définition a toujours raison ».
} 
ce qui leur est proposé. Par extension, ce monopole dégage les dirigeants du parti de tout contrôle immédiat et continu.

En second lieu, la participation par internet n'est en aucun cas obligatoire. Ainsi, cela revient à ériger en «volonté générale » (sinon des citoyens au moins des adhérents) ce qui n'est souvent que la position d'une minorité. À titre d'exemple, le Mouvement 5 étoiles revendique aujourd'hui plus de 135000 adhérents, mais les votes en ligne aux différents sondages et questionnaires régulièrement proposés recueillent en moyenne 30000 réponses. De même, en 2014, Pablo Iglésias devient secrétaire général de Podemos avec 88,6\% des votes, soit environ 95000 sympathisants. Un très beau score qu'il convient de relativiser au regard des plus de 250000 inscrits qui pouvaient participer à cette élection. Un écart similaire peut être constaté à l'occasion de la deuxième assemblée citoyenne de Podemos, qui se déroule en février 2017. Pablo Iglesias est reconduit dans ses fonctions avec 89,09\% des votes, soit 250000 voix, alors que le mouvement revendique plus de 450000 inscrits au moment du vote. Par ailleurs, et lors des deux scrutins, la totalité des membres du Conseil citoyen, qui ont été désignés formait une liste présentée et soutenue par l'équipe du secrétaire général.

De fait, il faut bien constater que soit l'utilisation de l'outil internet ne suffit pas forcément à remobiliser l'électorat (qui est pourtant initialement volontaire puisqu'il est adhérent du parti), soit que ce même outil ne touche pas l'ensemble de ce même électorat. Dans les deux cas, cela pose la question de l'impact de ces partis sur la vie politique.

\section{B. Un impact politique d'une durée incertaine}

Au regard de la jeunesse de ces mouvements, le temps n'est pas encore à un bilan critique, mais simplement à l'observation. Néanmoins, les premiers éléments tendent à démontrer que l'apport à la démocratie - ou à un renouveau démocratique - est parfois incertain (1) et, de fait, interroge sur la capacité de ces partis à perdurer (2).

\section{Un apport à la démocratie encore incertain}

Si le fonctionnement de ces partis semble malgré tout sinon plus démocratique au moins plus enclin à entendre la voix des citoyens, il reste à s'interroger sur l'apport de ces partis au débat démocratique. Et de ce point de vue, les positionnements sont très dissemblables et de nombreuses interrogations restent en suspens.

Ainsi, et pour des raisons différentes, il est aujourd'hui difficile de mesurer l'impact réel d'un Parti pirate ou encore d'une formation comme Podemos. Dans le premier cas, les élus sous cette étiquette restent trop marginaux pour quantifier leur influence. Les victoires récentes des partis pirate islandais et tchèque devraient constituer un bon test de ce point de vue. A priori, le Parti pirate islandais semble vouloir "s'institutionnaliser» puisque sans réellement d'états d'âme, et après avoir échoué à former une coalition gouvernementale, les élus Pirate se sont alliés au Mouvement des verts et de gauche ainsi qu'aux centristes du Parti du progrès pour former une opposition forte. 
Si l'antipartisme semble donc s'éloigner des préoccupations islandaises, il s'agit là d'une véritable chance (assez inédite pour le parti) d'influer sur la politique nationale.

Ce type de jeu d'alliances, dans le second cas, explique également les difficultés du Parlement espagnol à investir un gouvernement entre 2015 et 2016. Podemos est en partie responsable de cette période d'incertitude, notamment parce qu'il a refusé de s'allier avec le Parti socialiste ouvrier espagnol (PSOE). Ce refus a été l'occasion de voir poindre des dissensions, qui semblent perdurer, entre les deux figures emblématiques du parti : Pablo Iglesias et Íñigo Errejón. Si le premier reste fidèle à l'idée de mobilisation sociale du mouvement 15-M, le second prône un «changement de cap », avec une approche plus institutionnelle concrétisée par des alliances. Réélu au poste de secrétaire général par les adhérents en 2017, Pablo Iglésias va écarter de la direction du parti les promoteurs de ce dernier courant. Sergio Pascual (« secrétaire à l'Organisation de Podemos », considéré comme le numéro 3 du parti) est destitué dès le mois de mars 2016 et, Íñigo Errejón lui-même, voit disparaître de l'organigramme du parti sa charge de « secrétaire à la Politique de Podemos ». Il occupe désormais un poste moins «exposé » (et créé pour lui...) de «secrétaire de l'analyse stratégique et du changement politique de Podemos». Pour autant si cet affrontement interne est un aléa assez connu des partis politiques, il ne sera pas resté sans effet puisque lors des élections générales anticipées du 26 juin 2016, Podemos rejette tout rapprochement avec le PSOE mais s'allie, au sein d'une coalition dénommée Unidos Podemos, avec Izquierda Unida (gauche radicale) et Equo (parti écologiste). Il faut d'ailleurs reconnaître que ce compromis a été décidé par les adhérents du mouvement lors d'une consultation en ligne, dont le taux de participation a été de $30 \%$.

Ce refus d'alliance avec les autres partis politiques constitue le mode de fonctionnement du Mouvement 5 étoiles depuis son entrée au Parlement italien, parfois au prix de blocages très importants. À titre d'exemple, le refus du parti de prendre position (avec la majorité ou l'opposition) pour la désignation des juges constitutionnels a conduit la Consulta à travailler avec trois juges manquants pendant presque deux ans ${ }^{29}$. Ce refus presque systématique de «collaborer» avec les autres partis peut finalement s'avérer très révélateur: refuser permet d'éviter de choisir, donc de prendre position et finalement d'être éventuellement placé face à ses contradictions.

Ces incertitudes, concernant les trois partis pose forcément la question de leur devenir.

\section{Une capacité à perdurer difficile à évaluer}

L'image de mouvements populistes de ces partis essentiellement promus par internet est souvent renforcée par l'absence d'un projet politique clair.

Le Mouvement 5 étoiles cultive une ligne politique difficilement identifiable: défense de l'environnement, lutte contre la «malbouffe», sortie de la zone euro, opposition à l'immigration, ou encore réduction du temps de travail à vingt heures par semaine, etc. Ces quelques exemples

29 M. BARDIn, «La vie de la Cour constitutionnelle en $2015 »$, Annuaire international de justice constitutionnelle, vol. XXXI-2015, 2016, p. 796-799. 
constituent autant d'éléments rassembleurs, voire parfois «racoleurs», mais dont la cohérence d'ensemble reste discutable. En rassemblant aussi bien à gauche, à droite que chez les abstentionnistes, le Mouvement 5 étoiles est certes devenu un mouvement d'ampleur, mais qui donne l'impression de n'être que le « réceptacle du moment» des frustrations de l'électorat italien. Une frustration qui perdure pourtant puisqu'à l'aube du début de la campagne qui verra l'élection de la XVIII législature du Parlement italien, le Mouvement 5 étoiles est crédité par les derniers sondages de plus de $29 \%$ des intentions de vote ${ }^{30}$. En définitive, le positionnement «ni droite, ni gauche » des Grillini leur permet de rester aisément fidèles à la devise du mouvement: «Nous ne sommes pas un parti, nous ne sommes pas une caste: nous sommes des citoyens, point final».

Cette formule prend tout son sens grâce à internet et sa popularité doit d'ailleurs tout autant à l'image médiatique de son leader qu'à la stratégie numérique mise en œuvre par le co-fondateur du mouvement, aujourd'hui disparu. En effet, comme le rappelle le journaliste Alberto Di Majo ${ }^{31}$, Gianroberto Casaleggio a su persuader Beppe Grillo (qui, il faut le rappeler, avait l'habitude jusqu'au début des années 2000 de détruire sur scène des ordinateurs en affirmant qu'ils étaient «lourds et inutiles ») que l'intérêt démocratique d'internet était de supprimer la médiation entre les citoyens et l'exercice du pouvoir, en bref, les partis politiques.

Avec la disparition de Gianroberto Casaleggio (en 2016), Beppe Grillo perd son spin doctor, celui qui était sans doute le véritable idéologue du parti, ${ }^{32}$ mais aussi le gardien de l'image du mouvement ${ }^{33}$. Malgré l'intronisation de David Casaleggio, déjà associé au travail de son père, la « désorganisation » est parfois palpable au sein du parti, tout comme, de manière assez inédite, le non-respect de certains votes des adhérents qui risque de fragiliser le mouvement, comme le leadership, de Beppe Grillo.

Ainsi, au début de l'année 2016, et après de longues tractations, le mouvement renonce à soutenir l'union civile pour les couples de même $\operatorname{sex}^{34}$ (malgré une consultation en ligne et le vote favorable de $80 \%$ des participants) afin, selon les observateurs, d'éviter de s'aliéner les voix de droite en vue des élections municipales.

Dans le même sens, et justement à la suite des élections municipales de 2016, le parti découvre, au niveau local, les difficultés liées à l'exercice du pouvoir: dissensions au sein de la direction du mouvement, exclusion de certains membres à la suite de critiques sur la «démocratie interne»,

\footnotetext{
30 N. PAgnoncelli, «Sondaggi elettorali 2018, gli operai (e i giovani) votano M5S. Tra i pensionati primo il Pd, bene FI», Corriere della Sera, 27 janvier 2018, www.corriere.it.

31 A. Di MAjo, Grillo for president. Che cos'è, da dove viene e cosa vuole veramente il Movimento 5 Stelle, Rome, Editori Riuniti, 2012,319 p. 32 Voir notamment E. Greblo, Filosofia di Beppe Grillo. Il Movimento 5 stelle, Sesto San Giovanni, Mimesis Edizioni, 2011,118 p.

33 Un gardien parfois jugé, y compris en interne, comme «impitoyable et vindicatif», «contrôlant tout et tout le monde» comme l'affirmait Giovanni Favia, un conseiller régional d'Émilie-Romagne en 2012. Il concluait qu' «il n'existe pas de démocratie au sein du M5S ». Des propos qui conduiront à son exclusion définitive du parti quelques mois plus tard, «M5S, Favia: "Casaleggio prende per il culo tutti. Da noi la democrazia non esiste" », Il Fatto Quotidiano, 6 septembre 2012, www.ilfattoquotidiano.it.

34 C. Tzutzuiano, «Quand vouloir unir divise », La Lettre d'Italie, $\mathrm{n}^{\circ}$ 8, 2016, p. 11-12.
} 
remise en cause de la probité de proches collaborateurs de Virginia Raggi, élue premier édile de Rome en $2016^{35}$.

Il en va de même de Podemos qui va devoir se positionner au sein du Parlement espagnol et qui aura le plus grand mal à soutenir les revendications, parfois contradictoires des mouvements citoyens comme le 15-M, tout en formant des alliances politiques. En tant que troisième formation du pays, Unidos Podemos devra composer avec le psoe qu'elle le veuille ou non: rallier les voix du groupe socialiste pour ses projets et, de fait, en soutenir d'autres en retour.

Reste le modèle, plus international, proposé par le Parti pirate. Ce dernier apparaît clairement comme un «concept » plus que comme une force politique organisée. Le modèle existe grossièrement et - puisque, c'est le leitmotiv - il est «libre d'accès ». Il suffit pour cela d'observer les conditions d'adhésion au Parti pirate international: adhérer à la philosophie de l'association, utiliser le mot «pirate» dans sa dénomination et fonctionner sur des principes démocratiques ${ }^{36}$. Il semble difficile de structurer un mouvement - international de surcroît - sur cette base. Sans évolution, les résultats électoraux risquent de rester aussi bas qu'ils le sont, laissant ainsi le Parti pirate en dessous des seuils de financement.

D'ailleurs, au moins sur l'aspect internationaliste du mouvement, cette philosophie marque le pas. L'idée de coordination internationale des partis pirate nationaux semble vouée à l'échec, faute de finalité claire et de modalités précises de mise en œuvre de la coopération annoncée. Ainsi, le retrait de partis pirate importants comme ceux d'Australie, du Canada, d'Islande, du Royaume-Uni ou encore d'Irlande (pourtant un des pays fondateurs du Parti pirate international) démontre un désintérêt croissant. De même, l'«historique» Parti pirate suédois a quitté l'organisation dès 2015. Dans une moindre mesure, le Parti pirate européen, et sa vocation à coordonner les partis pirate lors des élections européennes, s'essouffle presque naturellement entre les échéances électorales.

Cela dit, ces aléas et ces tâtonnements ne sont pas sans rappeler ceux des mouvements écologistes des années 1970. Ces derniers s'associaient ponctuellement pour des actions, mais ne constituaient en aucun cas une véritable force politique malgré quelques participations à des élections locales. Il faudra attendre 1979, et la création du Mouvement d'écologie politique, pour qu'émerge une force politique. Il en est vraisemblablement de même pour le Parti pirate. S'il semble a priori mieux organisé que ne l'étaient les écologistes, la faiblesse de sa force de proposition tend à démontrer qu'il ne s'agit que d'une organisation de façade. Pour reprendre l'exemple des écologistes, ces derniers, une fois structurés et sans jamais renier leur attachement à la défense de l'environnement, ont développé l'idée d'une écologie politique visant à mettre en œuvre une société plus apaisée en luttant contre les déséquilibres et les inégalités. Aussi simpliste que paraisse l'«idéologie de départ», elle existe. Aujourd'hui, les seules idées lisibles du Parti pirate sont, d'une part, une volonté de réforme du droit de la propriété intellectuelle, mais dont les implications pratiques sont des plus floues et, d'autre part,

35 Cela dit, et fort heureusement, la gestion de la ville de Turin, par Chiara Appendino et la majorité municipale Cinque Stelle (élue le même jour que Virginia Raggi) semble plus sereine.

36 Art. 1.4.2-IV des statuts du Parti pirate international. 
la mise en œuvre d'un libre accès à la culture (qui n'est qu'une conséquence évidente de la première idée). Pour le reste, plus que des idées, le Parti pirate semble surtout avoir des revendications: beaucoup de critiques pour peu de propositions. Pour reprendre certaines expressions du programme, la «démocratie ouverte» qui permettra de «casser les structures pyramidales» et de «travailler en transversalité » afin de «coconstruire les politiques publiques avec les citoyens » nécessite quelques explications et des propositions plus précises que celles qui ont été jusqu'à maintenant formulées.

C'est finalement le lien entre tous ces mouvements politiques nés ou popularisés grâce à internet et aux technologies actuelles: le passage d'une activité de contestation à une véritable activité politique au sein des institutions, sans trahir les fondements qui ont conduit à leur émergence.

Il va de soi que pour ces formations, la détermination des orientations politiques doit être le fruit d'un consensus et même d'une validation par les adhérents. Cela dit, comme le démontre l'adaptation des partis politiques traditionnels, cette adhésion peut être largement encouragée.

\section{Numérique et campagnes électorales}

Simple conjoncture ou mouvement plus profond, le numérique a eu une influence certaine sur la dernière campagne présidentielle française. Il a, à cette occasion, généré de nombreux particularismes (A) et prouvé qu'il était un redoutable instrument de rationalisation des campagnes électorales (B).

\section{A. Le numérique comme générateur de particularismes?}

La campagne présidentielle française de 2017 a révélé aux yeux du grand public toute l'ampleur d'un aspect souvent ignoré de la «révolution numérique». Pour la première fois, l'ensemble des candidats a clairement assumé le fait de mener une campagne électorale où les agences de stratégie numérique se sont imposées comme un élément incontournable.

À l'échelle de la politique française, cette élection présidentielle multiplie les particularismes, en lien avec l'idée d'une démocratie connectée, qui conduisent à observer que ce scrutin n'était pas celui des partis.

En premier lieu, la mise en œuvre de primaires, comme élément de revitalisation et de recentrement de la participation partisane et même citoyenne s'est avéré un échec cuisant. D’une part parce qu'il a sans doute entériné - trop tôt ou trop tard - des choix de candidats qui se sont avérés finalement handicapants pour les grands partis. D’autre part, et le premier tour des présidentielles suffit à le démontrer, les meilleurs scores électoraux ont été obtenus à ceux qui ne se sont pas «encombrés » d'une telle joute.

Par ailleurs, et au moins pour partie dans l'esprit des mouvements citoyens espagnols et transalpins, cette élection présidentielle a vu prospérer, avec plus ou moins de succès, des primaires ou projets de primaire en dehors des partis politiques: La Vraie Primaire, LaPrimaire.org ou encore 
La Primaire des Français sont lancés tout au long de l'année 2016. Il convient malgré tout de rester mesuré quant au parallèle à établir avec les exemples étrangers précédemment cités. En effet, pour partie, certaines de ces primaires ne visaient pas forcément à désigner un candidat sans «historique politique». À titre d'exemple, La Primaire des Français prévoyait certes de mettre en compétition des personnalités de la société civile (par exemple, l'écrivain Alexandre Jardin avec le mouvement citoyen «Bleu Blanc Zèbre » puis «la Maison des citoyens ») mais également des mouvements comme «Génération Citoyen» (dirigé par Jean-Marie Cavada, député européen depuis 2004) ou encore «Le Rassemblement citoyen - Cap21» présidé par Corinne Lepage (ministre de l'Environnement de 1995 à 1997 et également élue au Parlement européen de 2009 à 2014).

En second lieu, cette campagne est sans doute la première élection où les candidats «sans parti » se sont montrés à leur avantage. Preuve d'une évolution des mœurs ou simple contexte où les partis traditionnels «ratent leur rendez-vous», les premiers signes ont été révélés par les «candidats à la candidature ». Ainsi, des personnalités aussi connues que Michèle Alliot-Marie ou Rama Yade fondent respectivement «Nouvelle France » et «la France qui ose », de très éphémères mouvements qui n’ont vocation qu'à soutenir la candidature à la présidence. Des mouvements éphémères puisqu'aucune des deux candidates ne recueillera le nombre suffisant de parrainages. Il en sera de même d'ailleurs pour la seule candidate issue d'une "primaire citoyenne », Charlotte Marchandise, désignée à l'issue de la consultation organisée sur le site LaPrimaire.org.

Ces embryons d'initiatives citoyennes pourraient laisser penser que le numérique n'est pas un vecteur suffisant, mais il en a été tout autrement puisque le numérique a bel et bien été au cœur de la campagne. Tous les candidats à l'élection présidentielle ont eu recours au numérique, et à titre d'exemple, Emmanuel Macron et Jean-Luc Mélenchon ont également amorcé la campagne présidentielle sans parti politique (de surcroît préexistant) en soutien. Sur le principe, une telle situation ne doit en aucun cas émouvoir puisque l'on a de cesse de répéter, depuis qu'elle a été instituée, que l'élection présidentielle est avant tout une élection personnelle.

L'émergence des partis politiques ne concerne donc pas que les mouvements dits citoyens, issus d'un mouvement de rejet ou de contestation. Dans ces deux cas, il s'agit bien de personnalités ayant occupé des responsabilités politiques et/ou détenu des mandats. Est-ce à dire que les partis politiques ou à défaut, les «politiques » ont «récupéré » à leur profit l'outil numérique? Certainement, et, il faut bien en convenir, avec une méthode dont le caractère démocratique peut être discuté. Là où les mouvements citoyens se sont avancés sur la scène politique à «visage découvert », les méthodes employées par les équipes de campagne des candidats relèvent plus d'une sorte de «profilage politique » visant à rationaliser la campagne électorale. Un «profilage» où le citoyen, et en l'espèce l'électeur potentiel, devient une donnée - et même une donnée personnelle - exploitable.

\section{B. Le numérique comme instrument de rationalisation}


Le lien entre l'outil numérique et les campagnes politiques n'est pas nouveau. Il intéresse les partis politiques français depuis la campagne présidentielle de Barack Obama en 2008 (considérée comme un modèle du genre ${ }^{37}$ ). Mais au-delà, ce sont les modalités d'utilisation de cet outil numérique qui constituent la véritable révolution.

Une révolution qu'il convient pourtant de juger avec recul tant la rationalisation de la campagne électorale passe par l'idée d'un profilage des électeurs (1) qui, poussé à son paroxysme, conduit même à ficher ces derniers (2).

\section{De la rationalisation de la campagne au profilage électoral}

Nombre de commentateurs ont « disséqué » la campagne numérique des candidats: le calendrier, les modalités de communication choisies, les moyens de susciter des attentes (et par extension de l'intérêt), l'utilisation experte des réseaux sociaux, etc. Une telle analyse s'avère passionnante, mais elle ne révèle qu'une partie de ce qui constitue, en France, une nouveauté politique. La véritable «révolution » en politique se situe en amont de la campagne: elle se situe dans les moyens utilisés pour déterminer les éléments qui permettront de mettre en œuvre une campagne numérique efficace.

Sans entrer dans des considérations trop techniques, des entreprises comme Blue State Digital (sur les campagnes de Barack Obama en 2008 et 2012 et celle de Jean-Luc Mélenchon lors de la dernière campagne présidentielle française) ou NationBuilder (notamment connue pour avoir été utilisée par Bernie Sanders aux États-Unis et par François Fillon, Bruno Lemaire ou encore Alain Juppé pour les primaires) sont des entreprises fournissant des «services de stratégie et de technologique numériques ». À cette appellation, DigitaleBox, un modèle plus français et plus récent, préfère celle de «gestion des réseaux sociaux et d'organisation de communautés».

Un exemple, désormais connu, tel que la plateforme de NationBuilder permet d'illustrer les mécanismes de cette stratégie numérique. Sous cette appellation emphatique se cache un «système de gestion de contenu » (souvent appelé cMs pour content management system) créé en 2009 aux ÉtatsUnis. Quelle que soit la structure «cliente», le modèle proposé repose sur quatre éléments: un site internet qui va servir de base au développement d'une communauté et permettre, une fois l'adhésion à cette dernière entérinée, d'organiser des collectes de fonds (le fundraising, traditionnel aux ÉtatsUnis), mais aussi et surtout de mettre en œuvre une communication plus efficace car plus ciblée.

Dans sa partie visible, la stratégie de communication se fonde sur une double acceptation: celle d'une adhésion, qui entraîne celle de la fourniture de données personnelles. Jusqu'ici, rien de très innovant, puisque le soutien des militants et des sympathisants est une donnée assez classique des campagnes politiques. L'outil numérique devient orwellien par la capacité de ces plateformes à optimiser les données personnelles fournies.

37 Voir I. FASSASSI, «Les effets des réseaux sociaux dans les campagnes électorales américaines », in cet ouvrage. 
Ainsi, NationBuilder (ou toute autre plateforme de ce type) permet, par l'intermédiaire d'une fonction «Match», d'automatiser la collecte de données personnelles issues de plusieurs réseaux sociaux (Facebook, LinkedIn, Twitter, etc.) grâce à une simple adresse e-mail. Par là même, au-delà de l'adhérent, c'est l'ensemble de son réseau social et de son activité numérique qui sont analysés. Mais l'outil va plus loin: il se «nourrit» plus largement encore puisqu'il recoupe ces informations avec d'autres types de données, publiques (celles de l'Insee, par exemple) ou privées (par l'achat ou la location de bases de données collectées par des entreprises). Ce ciblage électoral n'est qu'un premier pas puisque s'il peut sembler trop superficiel et dégagé de toute réalité pratique, d'autres instruments, par un nouveau croisement des données, vont optimiser cette approche numérique.

Ainsi, il est possible d'évaluer le profil de l'utilisateur (en l'espèce du sympathisant ou du militant en devenir). Une application comme Klout, permet de mesurer «l'activité numérique d'une personne et par là même sa capacité d'influence dans et sur les réseaux sociaux. De manière plus pragmatique encore, une application telle que Knockin permet de gérer un fichier de militants ainsi qu'une cartographie électorale et donc de mettre en place des campagnes de porte-à-porte rationalisées. Ajoutez à cela une application telle que Foursquare qui permet de géolocaliser les utilisateurs en temps réel et la somme d'informations recueillies, correctement traitée et utilisée, permet de mettre en œuvre une campagne électorale d'une rare précision. C'est sur ce dernier point que la différence existe. Il est un fait établi qu'une multitude de données sont collectées. Pour autant, le «talent» de ces agences de stratégie est bel et bien de savoir regrouper et exploiter des données (souvent brutes) afin de créer des interconnexions exploitables ${ }^{38}$ qui se matérialisent initialement par la constitution d'un fichier de sympathisants potentiels.

À l'heure des débats sur le fichage de masse et les atteintes à la vie privée, et alors que la fonction «Match» de NationBuilder est proposée aux clients français depuis 2013, elle n’a été désactivée en France qu'à la fin du mois de mars 2017 après une négociation entre la Commission nationale de l'informatique et des libertés (CNIL) et l'éditeur. Autant dire bien trop tard pour ne pas influer sur la campagne présidentielle.

En lien direct avec cette élection et au regard de l'usage quasiment systématique de ces nouveaux instruments par les candidats, il convient de constater qu'il ne s'agit pas là d'une «méthode miracle » conduisant irrépressiblement à la victoire. Cependant, il s'agit sans doute non seulement d'une nouvelle manière de conduire, et même de rationaliser, les campagnes électorales, mais aussi et surtout d'un «démultiplicateur d'effets». De ce point de vue, l'efficacité de la campagne d'Emmanuel Macron doit beaucoup aux outils numériques, tant en ce qui concerne la faculté d'atteindre puis de mobiliser un électorat potentiel que de s'assurer de l'efficacité des actions entreprises. Les outils numériques ont été utilisés pour «scénariser» la campagne: susciter l'attente, alimenter les interrogations et même la curiosité, et mettre en scène les réponses. En bref, appliquer des méthodes relevant du pur marketing.

38 Soit elle-même soit en sous-traitant avec des entreprises spécialisées dans le data wrangling (ou data munging), c'est-à-dire dans l'analyse et la gestion de données numériques. 
Mais cette nouvelle utilisation des outils numériques par des partis politiques, ou des personnes politiques, déjà médiatiquement exposés ne constitue pas, ou ne constitue peut-être déjà plus, une différence fondamentale avec les nouveaux mouvements issus du numérique. Si dès leur création, Podemos, le Mouvement 5 étoiles ou encore le Parti pirate ont mis en place une sélection des candidats par les adhérents grâce à un vote en ligne, certains d'entre eux, et plus particulièrement le parti italien, semblent, malgré tout, apprendre de la communication des partis dits classiques.

\section{Du profilage électoral au fichage des électeurs et des adhérents}

Ainsi, en 2016, le jour de la disparition de Gianroberto Casaleggio, l'antiparti lançait une plateforme collaborative baptisée Rousseau, désignée comme le sistema operativo (système d'exploitation ou système opérationnel) et décrite comme le «cœur battant» du mouvement. D’abord, très confidentielle, la plateforme accueille, dans une seconde version lancée à l'été 2017, l'essentiel des instruments de participation proposés aux adhérents et militants (l'accès leur est réservé, mais il est possible de parcourir certains éléments du site en tant qu'invité). A priori, le modèle proposé se rapproche de ce que le Parti pirate a mis en place dès sa création: consultation des propositions de loi en cours, vote sur les lignes directrices et les propositions politiques du mouvement (aux niveaux européen, national et régional) et même la possibilité de soumettre des propositions.

Il n'empêche qu'une fois dépassés ces instruments assez classiques pour les partis numériques, Rousseau réserve d'autres surprises qui démontrent que cette plateforme est autre chose qu'un instrument collaboratif.

Tout d'abord, et pour rester dans l'esprit affirmé, Rousseau n'offre pas plus de garanties que l'ancien site quant à la prise en compte finale des votes des adhérents. Il en va de même quant à la promotion des mesures proposées par les partisans : la sélection des sujets soumis à la discussion est tout aussi absconse qu'auparavant. La démocratie interne ne progresse donc pas et les dirigeants nationaux gardent la mainmise sur la direction politique du parti. Tout au plus, lors du lancement de la nouvelle version, les dirigeants ont-ils affirmé que certains votes seraient «certifiés », sans préciser par qui, ni comment...

Ensuite, cette plateforme propose des rubriques qui rappellent très clairement les cms proposés par des entreprises comme NationBuilder. Au premier chef, Rousseau prévoit la fameuse option de fundraising. Les adhérents sont appelés à faire des dons afin de compenser les « 42 millions d'euros de remboursement auxquels le Mouvement 5 étoiles a renoncé » en «refusant les financements publics» au titre de la législature actuelle. Toujours dans le pur esprit des CMs, Rousseau promeut et aide à mettre en place les différentes actions et manifestations du mouvement (avec là encore des appels à contribution dédiés).

Enfin, la section «e-learning» de la plateforme est plus intéressante encore pour un non-parti. Quelque 8000 documents constituent une sorte de vade-mecum pour l'éducation politique des adhérents. La rubrique, le plus souvent sous la forme de vidéos, offre ainsi des leçons sur la manière 
d'organiser une manifestation, de prendre la parole en public, sur ce que recouvre la fonction de conseiller municipal et même «comment devenir une voix» du parti ${ }^{39}$.

Cela dit, et comme souvent dans les liens qui unissent la politique et le numérique, le plus intéressant est ce qui n'est ni visible, ni revendiqué.

Ainsi, la gestion de la plateforme n'est pas assurée par le Mouvement 5 étoiles mais par l'Association Rousseau, qui en est également propriétaire. Le siège de cette association se trouve à la même adresse que la société Casaleggio Associati, la société de conseil en stratégie numérique basée à Milan et fondée par Gianroberto Casaleggio et son fils David. Ce dernier est le président (et le trésorier) de l'association et les autres administrateurs sont deux élus du Mouvement 5 étoiles (un conseiller municipal de Bologne et député européen) ainsi qu'un salarié de Casaleggio Associati.

Étant donné que cette société est à la base même de l'émergence du Mouvement 5 étoiles, il n'est pas injustifié, ni étonnant de voir cette dernière gérer (même indirectement) la plateforme Rousseau. Ce qui l'est plus en revanche, c'est l'étendue des données collectées et les modalités prévues pour adhérer au Mouvement 5 étoiles car il s'agit bien d'adhésion au mouvement.

Outre le fait, que la consultation du site conduit à accepter des «cookies $»^{40}$, l'accès illimité et la participation à Rousseau nécessitent une inscription et le lien proposé sur le site de l'association renvoie à la page d'adhésion du Mouvement 5 étoiles. Sous couvert d'une certaine autonomie, il n'est donc pas possible d'adhérer directement à cette association, il faut adhérer à celle du parti.

Par ailleurs, l'étendue des données semble pour le moins exorbitante. Outre les données d'usage (les nom, prénom, le sexe ou encore la date de naissance), un numéro de téléphone portable est exigé (avec mention que ce numéro doit être «personnel» et qu'il ne peut s'agir «d'un numéro partagé avec d'autres personnes»), mais aussi le numéro de carte d'identité ou à défaut de passeport (incluant la date de l'expiration éventuelle du document).

Enfin, les différents accords demandés pour finaliser l'inscription sont éloquents. D’une part, une acceptation sans réserve est exigée concernant les dispositions du statut de l'association (non pas celui de Rousseau mais celui de «l'association portant le nom de "Mouvement 5 étoiles"»), le code de déontologie du mouvement, l'organigramme et la légitimité des dirigeants du parti ou encore concernant les règles de sélection des candidats aux élections. D’autre part, les mentions relatives à la confidentialité conduisent à céder toutes ses données personnelles au Mouvement 5 étoiles puisqu'il faut autoriser «l'utilisation [des] données personnelles, y compris sensibles».

\footnotetext{
39 Une idée qui n'est pas inconnue des partis politiques français puisque LREM a mis en place des plateformes de formation et La France insoumise a inauguré au début de l'année 2018 son «école de formation» basée sur des cours magistraux mensuels (gratuits et dispensés devant un public) retransmis en ligne sur les comptes YouTube et Facebook du mouvement, ainsi que sur la mise en ligne de vidéos tutorielles consacrées à l'activité militante.

40 Il existe plusieurs types de «cookies», initialement, ils avaient vocation à gérer les sessions d'un utilisateur, ils ont ensuite été utilisés également pour personnaliser la navigation (publicités ciblées). Désormais, la collecte de données s'effectue grâce à des cookies «de pistage» qui permettent de suivre, et donc de connaître, les habitudes de navigation et plus globalement le comportement d'un utilisateur (sites visités, pages visitées sur un même site, requêtes exactes envoyées, etc.).
} 
Si la plateforme en elle-même ne propose rien d'illégal, certains éléments attirent clairement l'attention. Avec l'acception des «cookies» et surtout de «cookies tiers», les premiers permettent une analyse (donc un profilage) du comportement des utilisateurs et les seconds permettent à une entreprise («tiers») de collecter, d'analyser et de traiter ces données le plus souvent avec une finalité économique.

Le procédé est certes connu, puisque c'est le modèle économique des réseaux sociaux (une gratuité «de façade» puisque l'utilisateur «paye» avec ses données), mais il est plus ambigu lorsqu'il est utilisé par un «non-parti» qui fonde une large part de sa logique politique sur la nocivité des liens entre les mondes économique et politique...

De surcroît, le modèle utilisé, comme l'accès «extérieur » aux données collectées par le site, remet en cause la fiabilité de l'ensemble du système de participation en ligne. L'exemple le plus flagrant reste celui du secret du vote : il n'en est pas question dès lors que les administrateurs du site, mais aussi une ou plusieurs entreprises, peuvent relier cet acte à des informations très précises sur le participant. Avec de telles méthodes, la démocratie semble bien peu renouvelée.

La démocratie connectée, du point de vue des partis politiques peut s'avérer décevante, ou pour le moins insuffisante ou incomplète, sans devoir pour autant être minorée.

Il est une affaire entendue que le numérique a bouleversé la communication politique: sa forme comme son rythme mais aussi, et cela est plus regrettable, sa profondeur (un véritable programme politique s'expose difficilement en 140 signes sur Twitter...). Néanmoins, le numérique a aussi bouleversé la vie politique et plus particulièrement la vie des partis politiques.

Sur le principe, l'émergence de «partis numériques» a permis de bousculer les «habitudes» politiques. Ces partis conduisent sans doute une partie des électeurs à renouer avec la «chose politique » et ils leur permettent, au moins à un instant T, d'être ou d'avoir le sentiment d'être, entendu. Ils permettent de donner corps à une contestation qui prend souvent sa source dans un sentiment d'abandon ou d'impuissance. Et dans le même esprit, ils constituent une opportunité de reprendre possession d'une souveraineté qui, selon le sentiment qui anime ces partis, échappe aux citoyens parce qu'elle est confisquée par les élites politiques. Soutenu par ces partis, l'accès de nouveaux profils - de simples citoyens - aux mandats politiques constitue déjà une forme de victoire. À défaut de pouvoir se «débarrasser» des représentants, l'objectif de ces partis est donc de mettre en place des représentants... potentiellement plus «représentatifs». Les élus pirate, de Podemos, les Grillini et même une large partie des élus de la majorité présidentielle en France illustrent cette forme de renouveau. Seuls les technologies numériques et internet étaient à même de permettre un tel essor en un temps aussi réduit: de ce point de vue, «l'accélérateur de démocratie » qu'est le numérique est bien réel. 
Mais donner corps à une contestation, mettre en œuvre un mouvement d'ampleur et l'organiser, ne constitue qu'une partie de l'exercice du pouvoir. Et si le modèle évolue dans sa constitution, il évolue moins du point de vue des méthodes: des problèmes de centralisation de la décision aux batailles entre dirigeants sur l'idéologie du parti, toute organisation politique, afin d'être cohérente, se doit de rationaliser son fonctionnement. En cela, les nouveaux mouvements ressemblent parfois à s'y méprendre aux «anciens».

Il n'y a pas forcément de volonté desdits partis mais pourtant, cette reproduction des «travers » des partis politiques traditionnels semble presque inévitable: le Parti pirate semble relativement exempt de dysfonctionnements majeurs ou mettant à mal l'idéologie de départ, mais il faut bien reconnaître que les résultats ne sont pas au rendez-vous. En définitive, un tel exemple tend à prouver que le conflit, ou encore un minimum de centralisation de la décision, est indispensable à la compétition politique. Cela est sans doute en partie vrai : il n'est pas de parti sans leader. Le phénomène est devenu classique et, finalement, n'épargne pas les nouveaux mouvements. Cela dit, ce leadership peut être conduit de multiples manières. Il existe bien un débat idéologique, ou tout au moins sur le positionnement politique du parti au sein de Podemos mais en revanche, il n'en existe pas au sein du Mouvement 5 étoiles.

L'idéologie justement est au cœur des interrogations qui planent autour de ces partis et de l'engouement qu'ils suscitent. Entre idéologie trop marquée et absence d'idéologie, leur capacité à s'inscrire dans le temps semble inexorablement sujette à une forme de «normalisation ». L'exercice du pouvoir implique des positionnements politiques qu'il faudra assumer et qui situeront forcément ces formations sur l'échiquier politique national. En définitive, on ne saurait être un parti politique influent en restant un «non-parti ».

Mais l'idée de «normalisation», et cela est sans doute le plus dommageable, s'inscrit aussi dans les méthodes employées par certains «partis du numérique » pour s'imposer. La compétition politique est avant tout une compétition, et à l'image des partis traditionnels, la «chasse aux électeurs », par tous moyens (et notamment grâce aux technologies actuelles), intéresse tous les partis, fussentils les dépositaires désignés d'un hypothétique «renouveau politique». 


\title{
L'UTILISATION DES RÉ SEAUX SOCIAUX PAR LES PARLEMENTAIRES: INSTRUMENT DE REVITALISATION DE LA DÉMOCRATIE REPRÉ SE NTATIVE?
}

\author{
Priscilla JENSEL-MONGE ${ }^{1}$
}

L'idée que l'évolution des technologies de communication puisse être un instrument de progrès pour la démocratie n'est pas nouvelle. Ainsi, chaque avancée fait naître l'espoir d'une rénovation de nos systèmes politiques ${ }^{2}$ et c'est aujourd'hui internet qui porte les plus belles promesses ${ }^{3}$. Aucun phénomène d'exercice ou de conquête du pouvoir ne semble désormais pouvoir lui échapper. De la web campagne de Barack Obama en 2008 aux tweets retentissants de Donald Trump, en passant par la remise en cause des partis politiques traditionnels ${ }^{4}$, internet a aujourd'hui investi l'ensemble des strates du pouvoir. Les élections récentes, en France comme à l'étranger, ont démontré, s'il en était encore besoin, que le web joue un rôle central en matière de propagande électorale et de conquête du pouvoir ${ }^{5}$. La maîtrise de ce nouvel espace de communication apparaît comme un enjeu stratégique des candidats à une élection. Mais l'investissement d'internet ne se limite pas aux aspirants dirigeants et à ces périodes ponctuelles d'expression formelle de la démocratie. Les institutions communiquent également sur leurs activités à travers des stratégies qui sont de plus en plus élaborées et de plus en plus maîtrisées. Ce lien indissociable, qui unit donc le pouvoir à internet, est la conséquence de l'explosion rapide et massive de ce dernier au sein de la société. D'abord utilisé comme un outil d'informations, il est rapidement devenu un espace de communication et d'échanges et, par conséquent, un enjeu stratégique pour ceux qui détiennent ou souhaitent conquérir le pouvoir. Dans ce rapport qui lie internet et pouvoir, un outil occupe une place centrale ${ }^{6}$ les réseaux sociaux. Ces derniers peuvent être définis comme « des services web qui permettent aux individus de construire

\footnotetext{
1 Maître de conférences, Aix-Marseille Univ., Université de Toulon, Univ. Pau \& Pays Adour, CNRS, DICE, ILF-GERJC, Aix-en-Provence, France.

2 Th. VEDEL, «L'idée de démocratie électronique. Origines, visions, questions », in P. PERrineAu, Le désenchantement démocratique, La Tour d'Aigues, Éditions de l'Aube, 2003, p. 243. L'auteur identifie ainsi trois âges de la démocratie électronique (la machine à gouverner, la télédémocratie et la cyber-démocratie) qui correspondent chacun au contexte technique qui lui est contemporain.

3 Voir notamment Th. VeDEL, «La révolution ne sera plus télévisée. Internet, information et démocratie », Pouvoirs, 2006/4 (n 119), p. 41-54.

4 Sur cette question voir notamment M. BARDIN, «Les partis politiques face au numérique : entre adaptation stratégique et émergence de nouveaux mouvements », in cet ouvrage. Voir encore A. LITvinenko, «Social Media and Perspectives of Liquid Democracy on the Example of Political Communication of Pirate Party in Germany », in M. GAsco, Proceedings of the 12th European Conference on e-Government (ESADE, Barcelona), Reading, Academic Conferences and Publishing International Ltd, 2012, p. 403-408.

5 Sur l'influence des réseaux sociaux dans les campagnes électorales américaines voir notamment I. FASSASSI, «Les effets des réseaux sociaux sur les campagnes électorales américaines », in cet ouvrage.

6 D. CARdon, «Réseaux sociaux de l'Internet», Communications, 2011/1 (nº 88), p. 141.
} 
un profil public ou semi-public dans le cadre d'un système délimité, d'articuler une liste d'autres utilisateurs avec les lesquels ils partagent des relations ainsi que de voir et de croiser leurs listes de relations et celles faites par d'autres à travers la plateforme $»^{7}$. Il s'agit donc d'outils numériques qui permettent de se constituer un réseau personnel ou professionnel et qui fournissent des interfaces sociales, de dialogue et d'informations. Du mandat électif communal à la magistrature suprême, ce virage « $2.0 »$ n'a pas échappé aux gouvernants. Au-delà de l'aspect stratégique et de «marketing politique» qu'elle porte, l'utilisation d'internet par les gouvernants (ou les aspirants gouvernants) questionne la démocratie et son fonctionnement. En réduisant la distance entre les citoyens et les gouvernants et en plaçant l'orateur et l'auditeur sur un même espace de dialogue, internet se présenterait comme l'outil idéal de revitalisation de la démocratie. Il présenterait ainsi de nombreuses vertus et notamment celle d'instaurer une «véritable démocratie du débat ${ }^{8}$. Toutefois, si les évolutions technologiques portent un certain nombre de promesses, elles apportent également un certain nombre de dangers. «La promesse euphorique d'un monde d'échanges horizontaux et sans intermédiaires entre les individus» se confronte alors «à la menace catastrophique d'une disparition de la vie privée et d'une société de surveillance généralisée, dans laquelle chacun vivrait sous le contrôle et le regard des autres $»^{9}$. Cette réflexion générale sonne comme un avertissement qui doit inviter à demeurer prudent et à ne pas faire preuve d'un enthousiasme démesuré lorsque l'on s'interroge sur la capacité d'internet à rénover la démocratie, voire à instaurer une nouvelle forme de démocratie: la démocratie électronique.

La présente étude propose de s'intéresser à un aspect particulier de cette problématique : l'utilisation des réseaux sociaux par les parlementaires. Elle envisage ainsi, à partir d'une démarche empirique, d'engager une réflexion afin d'apprécier si cette pratique contribue à revitaliser la démocratie représentative en permettant notamment un dépassement de ses principaux défauts. Dans un premier temps, la démarche a consisté à élaborer un questionnaire ${ }^{10}$ qui a été envoyé à l'ensemble des parlementaires français, et dont l'objectif était de comprendre leur(s) motivation(s) dans l'utilisation des réseaux sociaux. Si une certaine convergence est apparue dans les réponses, force est de constater que peu de parlementaires se sont prêtés à l'exercice, ce qui constitue déjà un élément de réponse. Il a donc fallu doubler ce questionnaire d'une «infiltration» des réseaux sociaux des parlementaires. Cela a permis à la fois de compléter les résultats obtenus dans les questionnaires réceptionnés et de les confronter à la réalité de l'usage des réseaux sociaux par les parlementaires. Sans surprise, les résultats obtenus démontrent que les parlementaires français sont 2.0 et qu'ils utilisent largement les réseaux sociaux. Il apparaît ainsi évident que ces derniers sont devenus l'instrument privilégié

7 D. Boyd, N. Eutison, "Social Network Sites: Definition, History, and Scholarship", Journal of Computer-Mediated Communication, vol. 13, $\mathrm{n}^{\circ} 1,2007$, citées in D. CARDON, « Réseaux sociaux de l'Internet», op. cit., p. 141-142.

8 A. Melucci, Challenging Codes: Collective Action in the Information Age, Cambridge, Cambridge University Press, 1996, cité in Th. VedeL, «L'idée de démocratie électronique. Origines, visions, questions.», op. cit., p. 254.

9 D. CARDON, «Réseaux sociaux de l'Internet», op. cit., p. 145.

10 Ce questionnaire a été envoyé à l'ensemble des parlementaires de l'Assemblée nationale et du Sénat en septembre 2016. Sur ce questionnaire figuraient les questions suivantes (liste non exhaustive): Utilisez-vous les réseaux sociaux? Si oui, lesquels? Si non, pourquoi ?; Depuis quand et à quelle fréquence? Dans quels «domaines» les utilisez-vous (fonction de contrôle, fonction législative) et dans quels buts? De manière un peu plus générale, pensez-vous que les réseaux sociaux influencent d'une quelconque manière les rapports de force politique au sein et à l'extérieur des assemblées? 
de communication des parlementaires qui ont pleinement saisi les potentialités de cet outil. La présence des parlementaires sur les deux principaux réseaux sociaux (Facebook et Twitter) est significative $^{11}$. Les chiffres sont saisissants : 99,5\% des députés ont un compte Twitter et $58 \%$ des sénateurs. S'agissant de Facebook, les chiffres sont du même ordre. Ainsi, sous la XIV législature, quatre députés seulement ne disposaient ni de page, ni de compte Facebook. Les résultats démontrent par ailleurs que les réseaux sociaux ne sont pas un outil générationnel, contrairement peut-être à ce que l'on aurait pu penser, puisque les parlementaires les plus âgés y sont aussi présents. En revanche, là où la génération peut jouer c'est dans la gestion de ces réseaux sociaux. Certains parlementaires, le plus souvent les plus jeunes, gèrent eux-mêmes, totalement ou en partie, leurs réseaux sociaux. Si ce n'est pas un outil générationnel, ce n'est pas davantage un outil qui serait plus mobilisé par les hommes que par les femmes ou par les parlementaires de province par exemple. Sur le plan politique, on s'aperçoit ensuite que les réseaux sociaux sont utilisés par les parlementaires, qu'ils appartiennent ou pas à un groupe parlementaire, quelle que soit son appartenance politique (groupe majoritaire, groupe minoritaire, groupe d'opposition). Si les parlementaires non inscrits se montrent le plus souvent très actifs, l'activité du parlementaire sur les réseaux sociaux apparaît surtout liée à sa personnalité. La seule véritable différence qui subsiste concerne l'utilisation des réseaux sociaux entre l'Assemblée nationale et le Sénat. Les députés mobilisent en effet davantage les réseaux sociaux. Selon une première interprétation, on peut considérer que cela constitue un indice de ce que l'utilisation des réseaux sociaux a plutôt une vocation «électoraliste » puisqu'elle est davantage utilisée par les élus du suffrage universel direct. On peut aussi envisager que les sénateurs souhaitent conserver une certaine distance politique que ce soit à l'égard du Gouvernement ou des citoyens, ces deux interprétations n'étant d'ailleurs pas contradictoires.

Cette utilisation généralisée des réseaux sociaux par les parlementaires doit donc conduire à mener une réflexion plus profonde sur les espoirs et les dangers qu'elle porte pour la démocratie. Sous le prisme particulier que nous nous proposons d'étudier, un certain nombre de questions méritent alors d'être posées : internet et les réseaux sociaux favorisent-ils la délibération démocratique? Peuventils permettre l'instauration d'une certaine forme de démocratie participative ou pallier les carences de la démocratie représentative? Finalement, ce lien entre internet et pouvoir est-il en mesure de revitaliser la démocratie, voire d'instaurer une nouvelle forme de démocratie: la démocratie électronique? Dans un contexte de remise en cause des institutions et de crise de confiance politique, l'utilisation des réseaux sociaux par les parlementaires semble en effet se présenter comme un outil en mesure de reconnecter le citoyen à ses représentants et de dépasser les limites de la démocratie représentative (I). Mais en créant un lien virtuel, essentiellement descendant, il semble que cette pratique tende davantage à en accentuer les défauts (II).

11 Ces chiffres ont été obtenus après avoir recherché, pour chaque parlementaire, s'il disposait d'un compte ou d'une page Facebook et d'un compte Twitter. 


\section{Un dépassement des limites de la démocratie représentative}

Les axes autour desquels l'idée et les pratiques de la démocratie électronique se développent sont au nombre de trois: l'information, le débat et la participation à la décision ${ }^{12}$. En utilisant de manière régulière les réseaux sociaux pour communiquer sur leurs activités et échanger avec les citoyens, les parlementaires créent ainsi un lien permanent qui idéalise la notion de démocratie représentative et tend à réaliser une véritable « démocratie connectée».

\section{A. La permanence de la démocratie}

À travers l'utilisation des réseaux sociaux, le parlementaire élabore une véritable stratégie de communication. Les réseaux sociaux apparaissent ainsi comme un instrument de communication politique essentiellement individuelle. Ils sont d'abord utilisés comme une sorte d'agenda public, dans lequel on retrouve toutes les activités du parlementaire. Sans demander d'investissement de communication trop important, cela permet ainsi au parlementaire d'occuper en permanence l'espace virtuel. Bien que cette communication varie considérablement d'un parlementaire à un autre, cette pratique permet de montrer aux électeurs que le parlementaire de la circonscription est actif et qu'il méritera, lorsque le moment sera venu, sa réélection. Cette pratique a donc à l'évidence une finalité électorale.

Les réseaux sociaux sont également mobilisés par les parlementaires en tant qu'espace d'expression politique individuelle. De manière indirecte, c'est le cas lorsque le parlementaire communique sur les activités législatives ou de contrôle du Parlement. Ainsi, le parlementaire informe ceux qui le suivent sur les réseaux sociaux qu'il va poser une question au Gouvernement ou encore qu'il a déposé une proposition de loi ou un amendement. Même si cette communication reste limitée aux activités auxquelles le parlementaire participe, cela a pour avantage de relayer auprès des citoyens une partie des activités du Parlement, ce qui contribue à mieux le faire connaître. En attirant l'attention sur les activités de contrôle, cela tend également à accentuer le contrôle qui s'exerce sur le Gouvernement et d'une certaine manière à créer une nouvelle forme de responsabilité politique qui s'exerce non plus devant le Parlement mais devant le peuple, les parlementaires jouant alors un rôle d'intermédiaire. De manière plus directe, les réseaux sociaux vont servir d'espace au parlementaire pour exprimer une position individuelle sur l'action du Gouvernement, sur la politique internationale ou encore sur un fait d'actualité. Cette possibilité d'expression offerte par les réseaux sociaux présente de nombreux avantages pour les parlementaires. Elle permet ainsi de contourner certaines contraintes liées au fonctionnement du Parlement. Les réseaux sociaux offrent la possibilité à chaque parlementaire de pouvoir réagir et de prendre position sur un fait politique d'actualité, ce que ne permettent pas les règles contraignantes d'attribution du temps de parole au sein du Parlement. Cela permet alors de soutenir la théorie de la souveraineté nationale selon laquelle chaque parlementaire représente

12 Th. VEDEL, «L'idée de démocratie électronique. Origines, visions, questions. », op. cit., p. 252. 
au Parlement la Nation tout entière ${ }^{13}$, et de contourner les inégalités liées au fonctionnement du Parlement qui tient davantage compte de l'importance politique du groupe auquel le parlementaire appartient au sein de l'assemblée. Par ailleurs, la logique collective qui domine le fonctionnement des assemblées parlementaires, et qui s'est encore accentuée depuis la révision constitutionnelle du 23 juillet $2008^{14}$, est dépassée par cette capacité d'expression individuelle puisque les réseaux sociaux offrent au parlementaire la possibilité de prendre position en dehors de son groupe. Enfin, on relèvera que l'expression individuelle des parlementaires sur les réseaux sociaux leur offre une double liberté de ton et de sujets, ce qui, encore une fois, est rarement le cas dans les assemblées parlementaires. Pour les parlementaires, l'utilisation des réseaux sociaux se présente donc comme un outil de communication qui offre à la fois plus d'égalité et plus de liberté.

L'utilisation des réseaux sociaux par le parlementaire a donc pour première conséquence de créer un lien, d'une nature particulière, entre les représentants de la Nation et les représentés en dehors des périodes électorales. Elle tend ainsi à offrir à la démocratie la permanence qui lui fait défaut et qui ne tend à s'exprimer formellement qu'au moment des élections. Si l'on considère que l'un des principaux défauts de la démocratie représentative est la distance qu'elle crée entre les gouvernants et les gouvernés, force est de constater que les réseaux sociaux ont cet avantage de réduire cette distance puisque se crée un lien permanent, et plus seulement au moment des échéances électorales, entre ceux qui exercent la souveraineté et ceux qui en sont les détenteurs. Ce lien permanent est aussi direct puisqu'à travers ces interfaces virtuelles, le parlementaire interagit avec les citoyens sans intermédiaire. Pour le parlementaire, cela permet de maitriser sa communication. Pour le citoyen, c'est un moyen rapide et direct de s'adresser à son représentant. Plus encore que le caractère direct du lien qui est créé, c'est l'horizontalité qui est intéressante dans une perspective de revitalisation de la démocratie représentative. En effet, en intervenant sur une même interface de dialogue, les réseaux sociaux offrent un espace sur lequel parlementaires et citoyens échangent dans une relation en apparence égalitaire. Cela tend donc à briser la dimension verticale qui caractérise traditionnellement leurs relations.

Ce lien quasi-institutionnel, permanent et direct, qui s'établit entre les représentants et les représentés présente cet autre avantage d'être à double mouvement. Il est à la fois descendant et ascendant. Dans sa dimension descendante, la communication des parlementaires sur les réseaux sociaux contribue à favoriser la transparence, exigence inhérente à la démocratie. Elle permet de diffuser de nombreuses informations, en dépassant les difficultés techniques ou les coûts que pourrait engendrer un autre mode de diffusion. Les parlementaires peuvent ainsi, par exemple, partager un lien vers le rapport d'une commission d'enquête ou vers un dossier législatif et ainsi intéresser davantage les citoyens au travail parlementaire, et plus largement au fonctionnement de la démocratie. Cet effort d'informations des citoyens constitue aujourd'hui une carence dans les démocraties

13 Voir en ce sens par exemple la décision du Conseil constitutionnel n ${ }^{\circ} 2004-490$ DC du 12 févr. 2004, Loi organique portant statut d'autonomie de la Polynésie française, cons. 14, 702 mars 2004, p. 4220, Rec., p. 41.

14 Qu'il nous soit permis de renvoyer à notre thèse de doctorat, P. Monge, Les minorités parlementaires sous la Ve République, Paris, Dalloz, 2015, p. 113 s. 
libérales actuelles ${ }^{15}$. En renforçant les informations disponibles, et en tendant par conséquent vers la réalisation d'une démocratie de citoyens éclairés, la communication des parlementaires sur les réseaux sociaux apparaît donc essentielle. Ce mouvement descendant permet par ailleurs, en réaction, un mouvement ascendant, qui part des citoyens. Les réseaux sociaux leur offrent en effet le moyen de s'émanciper d'une relation trop verticale et passive ${ }^{16}$ et, par conséquent, de prendre une part plus active à l'exercice de la démocratie. Lorsque le parlementaire communique sur les réseaux sociaux, il se livre à l'échange et à la critique. En scrutant les paroles et les actes des parlementaires et, le cas échéant, en les interpellant sur les réseaux sociaux, les citoyens obligent alors les représentants à rendre des comptes devant ceux au nom desquels ils exercent la souveraineté. Même les citoyens qui ne prennent pas part aux discussions peuvent, dès lors qu'ils sont connectés, être spectateurs d'un échange d'arguments contradictoires. Cela crée donc un nouveau canal d'informations et de surveillance pour les citoyens qui ne se contentent plus d'élire leurs représentants. Sans remettre en cause la nature représentative du mandat, cela tend à instaurer une forme de responsabilité politique individuelle et à conférer au concept de représentation son sens le plus fort. Si pour Pierre Rosanvallon, les activités de surveillance et de dénonciation sont à la base de la contre-démocratie ${ }^{17}$, alors l'utilisation des réseaux sociaux contribue, d'une certaine manière, à sa réalisation ${ }^{18}$ ou, plutôt, à celle d'une contre-démocratie électronique.

\section{B. La poursuite de l'idéal délibératif}

Si l'utilisation des réseaux sociaux par les parlementaires est en mesure de créer un lien permanent entre les représentants et les représentés, et de redonner ainsi tout son sens au concept de représentation, elle est également en mesure de pallier l'une des dérives durables de nos régimes politiques contemporains: l'affaiblissement du Parlement et de sa capacité délibérative. Ainsi, la logique exécutive qui gouverne le fonctionnement de nos institutions sous la Vépépublique a progressivement conduit à réduire le Parlement à une fonction de contrôle qui serait dès lors devenue sa fonction essentielle. Si le contrôle parlementaire est «la fonction parlementaire par excellence ${ }^{19}$, puisqu'il se trouve aux fondements mêmes du régime parlementaire, il est également devenu un discours de justification de la disparition du Parlement législateur. Dès lors, si la délibération ne trouve plus véritablement de place dans nos assemblées politiques, le débat public se déplace naturellement vers d'autres espaces et notamment vers les réseaux sociaux ${ }^{20}$. Ainsi, dès les années 1990, «le cyberespace est perçu comme un lieu de dépassement des systèmes politiques où peut se forger une contre-société qui absorbera en retour le vieux monde politique réel $»^{21}$. Les réseaux sociaux, et plus largement internet, seraient ainsi devenus une nouvelle agora électronique ${ }^{22}$ comparable à l'espace

\footnotetext{
Th. VEDEL, «L'idée de démocratie électronique. Origines, visions, questions. », op. cit., p. 250.

D. CARDON, «Réseaux sociaux de l'Internet», op. cit., p. 147.

P. Rosanvallon, La contre-démocratie. La politique à l'âge de la défiance, Paris, Seuil, 2006.

P. Flichy, «Internet et le débat démocratique», Réseaux, 2008/4, nº 150, p. 179.

$\mathrm{Ph}$. LAUvAuX, «Le contrôle, source du régime parlementaire, priorité du régime présidentiel», Pouvoirs, 2010, n 134 , p. 35.

A. Delcambre, A. Piquard, «Facebook, faux ami de la démocratie », Le Monde, 3 novembre 2016.

Th. VEDEL, «L'idée de démocratie électronique. Origines, visions, questions. », op. cit., p. 246.

Voir notamment P. Flichy, L’imaginaire d'Internet, Paris, La découverte, 2001.
} 
public habermassien ${ }^{23}$. Ils permettraient de réaliser une véritable démocratie du débat en créant un « espace public vigoureux et ouvert, à même d'accueillir l'expression d'idées multiples, de demandes qui ne peuvent se manifester dans le cadre institutionnel et rigide de la démocratie représentative $»^{24}$. En effet, les réseaux sociaux apparaissent comme un lieu ouvert à une diversité d'idées et à une multitude d'acteurs ${ }^{25}$, y compris par exemple aux citoyens les moins actifs. Ils constituent donc une porte d'entrée pour tous au débat public. Ils tendent ainsi vers l'idéal délibératif dans lequel chacun pourrait prendre part au débat public et, in fine, participer à la prise de décision politique, qui devient alors légitime ${ }^{26}$. C'est donc par nature le lieu idéal pour échanger de manière contradictoire puisque tous ceux qui le souhaitent peuvent prendre part au débat, à condition de se trouver sur ces plateformes. C'est aussi un lieu qui transcende les frontières culturelles, sociales ou géographiques et qui partage avec la démocratie les mêmes principes de base que sont la liberté et l'égalité. En élargissant et en stimulant ainsi la discussion et en mettant à la disposition des citoyens un grand nombre d'informations ${ }^{27}$, l'utilisation des réseaux sociaux par les parlementaires apparaît alors en mesure de revitaliser le débat public et la démocratie.

Dans ce nouvel espace, l'utilisation des réseaux sociaux va permettre au parlementaire de jouer un rôle à la fois de catalyseur, de modérateur et de médiateur du débat public. En partageant des contenus politiques, qu'ils touchent ou non aux activités du Parlement, le parlementaire provoque une réaction chez ses lecteurs et permettre au débat de s'engager sur un sujet de son choix. Il peut alors y prendre part ou bien demeurer spectateur de celui-ci. Lorsqu'il souhaite s'engager dans le débat, la difficulté tient à ce qu'il doit argumenter afin que le débat demeure constructif. En effet, le danger provient de ce que les règles de l'espace public, qui est un espace autonome, sont très différentes des règles applicables au sein des assemblées parlementaires. Sa fonction expressive peut alors conduire le parlementaire à jouer un véritable rôle de modérateur du débat, à l'image de ce que font les présidents des assemblées parlementaires. Il dispose en effet de cadres de référence et d'interprétation en matière politique qui vont lui permettre de structurer, voire d'enrichir, le débat. L'intérêt de provoquer et de prendre part au débat public est multiple et, tout comme le lien qu'il crée entre les représentants et les représentés, il connaît un double mouvement dans lequel le parlementaire fait office de médiateur. En servant de point de contact entre l'espace public virtuel et les assemblées, le parlementaire permet d'abord une externalisation du débat. Il partage ainsi un texte de loi à l'ordre du jour ou encore un amendement ou une proposition de loi qu'il a déposé. L'intérêt est alors double. Cela lui permet d'abord d'apprécier la réaction de l'opinion publique pour le texte ou pour son initiative. Cela lui offre également la possibilité de puiser des arguments ou d'affûter la défense de son texte lorsqu'il aura la parole dans l'hémicycle. D'une certaine manière, cela permet également, à travers le parlementaire, de faire pénétrer la parole citoyenne dans les assemblées

23 Voir notamment H. Rheingold, The Virtual Community. Homesteading on the Electronic Frontier, New York, Harper Perennial, 1994.

24 A. Melucci, Challenging Codes: Collective Action in the Information Age, Cambridge, Cambridge University Press, 1996 cité in Th. Vedel, «L'idée de démocratie électronique. Origines, visions, questions.», op. cit., p. 254.

25 Les questionnaires ont notamment révélé que les parlementaires bloquent rarement des personnes sur leurs réseaux sociaux et lorsque c'est le cas c'est en réaction à des échanges agressifs ou irrespectueux.

26 B. MANin, «Volonté générale ou délibération? Esquisse d'une théorie de la délibération politique », Débat, 1985, n³3, p. 77.

27 Th. Vedel, «L'internet et la démocratie: une liaison difficile», in P. Perrineau, L. Rouban, La démocratie de l'entre-soi, Paris, Presses de la Fondation nationale des sciences politiques, 2017, p. 76 et s. 
représentatives. De manière plus générale, y compris en dehors de toute forme de débat, cela permet aussi de capter les préoccupations des citoyens connectés ${ }^{28}$ et de les restituer devant la représentation nationale, notamment dans les activités de contrôle. Dès lors, il ne faut pas minimiser l'importance de la médiation opérée par le parlementaire dans ce cadre. Cette fonction de médiation lui permet de dépasser l'expression des intérêts particuliers qui vont s'exprimer, plus qu'ailleurs encore sur ce type de support de communication. Il peut ainsi les rassembler et dégager, sinon l'intérêt général, au moins un intérêt commun puisque dans tout processus démocratique, à la diversité des opinions doit nécessairement succéder l'unité de la décision.

Les espoirs portés par internet et notamment par l'utilisation que font les parlementaires des réseaux sociaux sont donc nombreux: plus d'égalité, plus de liberté, permanence du lien entre les représentants et les représentés, restitution des préoccupations de la société civile et de la parole citoyenne, réalisation de l'idéal délibératif, etc. Mais ce nouvel idéal démocratique n'est pas exempt de dangers et soulève un certain nombre de difficultés qui démontrent que le risque pour les parlementaires qui utilisent les réseaux sociaux est d'accentuer les défauts de la démocratie représentative.

\section{Une accentuation des défauts de la démocratie représentative}

La démocratie du débat que prétendent favoriser internet et les réseaux sociaux apparaît en réalité comme un leurre en raison de la nature même de ces supports de communication. Ainsi, ce qui constitue la force de l'idée de démocratie électronique en constitue également l'une de ses principales faiblesses. Les réseaux sociaux apparaissent alors comme de faux-amis de la démocratie ${ }^{29}$ susceptibles de renforcer la crise de confiance qui affecte déjà le régime représentatif.

\section{A. La fiction délibérative}

La principale critique qu'il est possible d'adresser à l'idée qu'internet et les réseaux sociaux seraient devenus une nouvelle agora électronique est son incapacité à susciter un véritable débat démocratique. S'il est incontestable que le débat public est désormais sur ces espaces, et qu'il s'est encore accentué avec la présence des gouvernants, force est de constater qu'il ne respecte pas les exigences démocratiques d'un débat, à la différence des médias traditionnels. Il n’y a pas d'équilibre dans les sujets qui sont proposés. Le principe du contradictoire n'est pas respecté. Les différents points de vue ne sont pas toujours entendus ${ }^{30}$. En dépit du rôle de médiation joué par les parlementaires sur les réseaux sociaux, les échanges qui s'y tiennent apparaissent alors davantage comme des «monologues interactifs ${ }^{31}$ qui se traduisent rarement par des discussions argumentées et construites susceptibles d'aboutir à une position commune. Au contraire, la virtualité des échanges, qui protège chaque in-

\footnotetext{
28 D. CARDON, «Réseaux sociaux de l'Internet», op. cit., p. 142. L'auteur démontre que les réseaux sociaux s'enracinent dans une expérience beaucoup plus proche des attentes et des pratiques ordinaires des utilisateurs. C'est un espace familier et navigable où les personnes exposent les traits de leur identité.

29 A. Delcambre, A. Piquard, «Facebook, faux ami de la démocratie», op. cit.

$30 \quad$ Idem.

31 M. Dumoulin, «Les forums électroniques : délibératifs et démocratiques? », in D. MoniÈRE, Internet et la démocratie. Les usages politiques d'Internet en France, au Canada et aux Etats-Unis, Québec, Monière et Wollank éditeurs, 2002, p. 156.
} 
terlocuteur derrière un écran, tend à durcir et à radicaliser les prises de position et à réduire le débat à une sorte de marché aux idées ${ }^{32}$. L'égalité et la liberté, qui sont par nature attachées aux réseaux sociaux, n'ont donc pas pour conséquence d'instituer un espace pluraliste où les différents points de vue s'expriment de manière argumentée en vue d'emporter la conviction de son interlocuteur. C'est simplement un espace ouvert à tous où l'expression est totalement libre.

Plusieurs raisons expliquent cette incapacité des réseaux sociaux à engager un véritable débat démocratique. Cette déception est d'abord liée au fait que les débats qui s'y tiennent n'ont pas vocation à aboutir à une décision politique. S'ils constituent une sorte d'exutoire pour des citoyens en manque d'expression politique, ils ne sont pas en mesure de tendre vers l'idéal de démocratie directe souhaitée par Rousseau et de contourner les difficultés liées à l'impossible réalisation d'une démocratie directe à grande échelle ${ }^{33}$. Ils demeurent des outils informels de participation des citoyens au débat public mais, pour des raisons évidentes, ils ne peuvent être envisagés comme des instruments formels de la prise de décision politique. Les débats qui se tiennent sur les réseaux sociaux sont donc dispensés de responsabilité de la décision collective. Contrairement au Parlement qui exprime la souveraineté nationale, les réseaux sociaux n'expriment qu'une partie des préoccupations de ceux qui sont les plus actifs et, par conséquent, loin d'être une image fidèle de la société, ils n'en sont qu'un miroir déformant.

Le mode de fonctionnement de ces espaces constitue, ensuite, une autre explication de cet échec du débat démocratique virtuel. Les réseaux sociaux aboutissent à la création d'un cercle fermé entre des personnes qui partagent les mêmes opinions politiques ${ }^{34}$. La logique réticulaire repose en effet sur la mise en place progressive de liste d'amis. Cette remarque vaut également, même si elle supporte quelques tempéraments, pour le parlementaire qui utilise les réseaux sociaux. Les citoyens connectés s'orientent en effet davantage vers les personnalités politiques avec lesquelles ils partagent les mêmes idées. De manière plus rare, ils peuvent également faire le choix de suivre le parlementaire élu dans le cadre de leur circonscription ou un parlementaire médiatique. Cela conduit alors à une double situation: soit les échanges sont très consensuels entre personnes qui partagent les mêmes opinions et qui les confortent mutuellement; soit les échanges sont très agressifs, voire injurieux, et les positions se radicalisent. En définitive, les réseaux sociaux n'apparaissent pas comme un lieu de confrontation pluraliste des opinions mais davantage comme un espace de confortation de ses propres idées. Ce constat se vérifie également s'agissant du parlementaire puisque celui-ci ne va chercher sur les réseaux sociaux que les arguments qui confortent sa position au détriment des autres. Il faut rappeler à ce titre l'idée fondatrice d'internet. C'est un espace qui a été pensé pour mettre en relation des personnes présentant des intérêts communs ${ }^{35}$, et non l'inverse. Paradoxalement, cet espace, qui est par nature ouvert à tous, contribue donc à créer un entre-soi qui enferme les individus dans une bulle idéologique, là où l'espace public réel conduit des personnes d'opinion politique différente à

32 Th. VEDEL, «L'idée de démocratie électronique. Origines, visions, questions. », op. cit., p. 264.

33 Ibid., p. 259.

34 Voir notamment A. Lev-ON, B. MANin, «Internet: la main invisible de la délibération», Esprit, 2006, n 5, p. 195-212; P. FLICHY, «Internet et le débat démocratique », op. cit., p. 168 et s.

35 P. Flichy, «Internet et le débat démocratique », op. cit., p. 163-164 
se rencontrer et à échanger. Si cet enfermement, qui est à la fois social et algorithmique ${ }^{36}$, n'est pas nouveau et existait déjà avec les médias traditionnels - avec la presse notamment -, il est d'autant plus inquiétant qu'il est le plus souvent subi, voire inconscient avec les réseaux sociaux ${ }^{37}$.

On relèvera également que la fiction délibérative électronique repose sur l'idée d'un citoyen nécessairement actif et éclairé et postule un état de politisation permanent. Or, être présent sur les réseaux sociaux n'implique pas nécessairement une volonté de prendre une part active au débat politique. Il est évident que ceux qui décident de suivre un parlementaire s'inscrivent déjà dans une démarche politique. Pour autant, cela témoigne le plus souvent d'une volonté d'être un simple spectateur de l'action et de l'activité du parlementaire. C'est uniquement lorsqu'un contenu engendrera une charge émotionnelle chez son lecteur, que celui-ci fera le choix de prendre part au débat, avec les risques que cela implique. En définitive, il ne suffit pas d'être sur les réseaux sociaux pour que ceux-ci deviennent un instrument de débat démocratique.

Incapable de produire un échange constructif, l'utilisation des réseaux sociaux par les parlementaires ne semble pas davantage en mesure de restaurer la confiance politique et d'apporter plus de sérénité à la démocratie. L'illusion d'horizontalité qu'elle tend à créer et la virtualité du lien qui s'établit risquent au contraire d'accentuer la crise qui affecte aujourd'hui le système représentatif.

\section{B. Le risque de renforcement de la crise de confiance politique}

La désillusion provient d'abord d'un double problème de temporalité au regard de la décision politique. Avec les réseaux sociaux, le temps apparaît à la fois trop court et trop long. Il est d'abord trop court parce que les attentes et les inquiétudes se propagent à la vitesse numérique. La logique émotionnelle qui domine ces espaces, et qui conduit à commenter ou à partager des contenus qui provoquent un sentiment fort chez son lecteur ${ }^{38}$, crée une forme d'empressement qu'il est aisément possible, pour les gouvernants, de mesurer sur les réseaux sociaux. Or, cela tend à renforcer l'une des dérives de nos sociétés actuelles qui est celle de la législation par l'émotion, voire même de la constitutionnalisation par l'émotion. Il apparaît néanmoins essentiel d'offrir à la démocratie le temps de respiration nécessaire à la prise de décision politique, lequel ne saurait correspondre au temps numérique. Le temps devient trop long, à l'inverse, si l'on considère que l'utilisation des réseaux sociaux doit permettre au débat de s'extraire des assemblées parlementaires, de vivre sur la Toile pour finalement revenir enrichi dans les hémicycles. Cette vision un peu idyllique se heurte à l'empressement dont font de plus en plus preuve les gouvernants. La pression populaire les oblige en effet à produire des résultats rapides ce qui les conduit à adopter des décisions dans des délais qui sont de plus en plus courts. Ainsi, le 6 octobre 2016, dans un colloque qui avait pour thème « Refaire la démocratie », le Président de la République François Hollande a annoncé vouloir refondre complètement la procédure législative «parce qu'il existe un décalage de plus en plus flagrant entre le temps exigé

36 Sur la question de l'enfermement algorithmique, voir notamment A. Delcambre, A. PiQuARD, «Facebook, faux ami de la démocratie », op. cit.; Th. VEDEL, «L'internet et la démocratie : une liaison difficile», op. cit., p. 77 et s.

37 A. Delcambre, A. Piquard, «Facebook, faux ami de la démocratie », op. cit.

38 Idem. 
par le mode d'élaboration de la loi et la rapidité attendue par les citoyens ${ }^{39}$. Si celui-ci n'a pas eu le temps d'engager une telle réforme, son successeur à la magistrature suprême semble s'inscrire à son tour dans un processus d'accélération de la prise de décision politique.

Le renforcement de la crise de confiance politique peut ensuite provenir du risque important de manipulation qui existe sur les réseaux sociaux. Ce danger concerne d'abord les informations diffusées et le problème de leur évaluation. Elles impliquent en effet de faire preuve de vigilance et de disposer d'une certaine distance critique à leur égard, ce qui constitue l'une des principales limites des réseaux sociaux en tant qu'outil d'informations. La logique émotionnelle et l'enfermement idéologique conduisent à sélectionner, de manière parfois inconsciente, celles qui nous confortent et à les considérer comme avérées. Ainsi, la popularité des contenus est souvent plus forte que leur véracité ce qui induit que les mensonges et les rumeurs sont autant lus que les faits avérés ${ }^{40}$. Le risque de manipulation concerne ensuite les acteurs. Les réseaux sociaux offrent en effet une identité virtuelle derrière laquelle il est impossible de connaître l'identité réelle de son interlocuteur. Il est donc difficile de savoir si l'on échange avec un citoyen lambda ou avec une structure beaucoup plus organisée susceptible de manipuler l'opinion et de faire pression sur le parlementaire. Par ailleurs, et de manière générale, l'utilisation des réseaux sociaux par les parlementaires contribue à accentuer le phénomène du consumérisme politique, en captant les préoccupations et les habitudes des individus qui s'exposent de plus en plus ${ }^{41}$.

L'utilisation des réseaux sociaux par les parlementaires risque enfin de dévaloriser encore davantage la fonction parlementaire, de créer une confusion entre la personne qui s'exprime et la fonction qu'elle exerce et d'accentuer le phénomène d'hypersonnalisation du pouvoir. L'enjeu pour le parlementaire est alors de réussir à donner l'illusion d'une relation de proximité tout en conservant la distance nécessaire à l'exercice de sa fonction. Or, pour de nombreuses raisons, cela n'est pas toujours le cas. Force est donc de constater que les réseaux sociaux ont permis à la parole politique de se libérer et que les propos tenus par les parlementaires ne sont pas toujours à la hauteur de ce que l'on peut attendre d'un dépositaire de la souveraineté nationale ${ }^{42}$. La difficulté provient du support de communication lui-même puisque les réseaux sociaux, et notamment Twitter, ont vocation à diffuser des messages courts, souvent exagérés, des réactions spontanées, sur un ton généralement badin et familier, afin de pouvoir se démarquer de la masse d'informations diffusées quotidiennement. Cela crée alors une confusion dans laquelle la parole publique et la parole privée se confondent ${ }^{43}$, ce qui ne contribue pas à restaurer l'image des représentants de la Nation et du Parlement.

39 Déclaration de M. François Hollande, Président de la République, sur la démocratie et le fonctionnement des institutions de la V ${ }^{\mathrm{e}}$ République, Colloque «Refaire la démocratie », Paris, 6 octobre 2016.

40 A. Delcambre, A. Piquard, «Facebook, faux ami de la démocratie », op. cit.

41 D. CARDON, «Réseaux sociaux de l'Internet», op. cit., p. 141.

42 Voir par exemple le tweet du député Philippe Vitel du 2 avril 2014: «Et Valérie [Trierweiler], elle n’a pas droit à un ministère comme Ségolène. Au nom de la justice du plumard». Il semble difficile de considérer que le parlementaire donne ici son avis sur la composition du gouvernement.

43 P. Flichy, «Internet et le débat démocratique», op. cit., p. 169. 
En utilisant de manière régulière les réseaux sociaux, les parlementaires semblent avoir réussi à mettre en relation deux espaces que tout oppose: le Parlement, où la logique majoritaire s'exprime pleinement et où la parole est limitée par un temps parlementaire toujours plus réduit; l'espace public virtuel dominé par la liberté et l'égalité. À bien des égards, cette pratique, aujourd'hui largement répandue, contribue à donner un nouveau souffle à la démocratie. Mais cette conclusion ne vaut que si l'on fait preuve de mesure, voire de modestie, quant à la capacité d'internet à panser les maux du régime représentatif. Les réseaux sociaux ne permettent pas d'instaurer une véritable démocratie électronique. Au même titre que la démocratie directe souhaitée par Rousseau, la démocratie connectée ne semble pas en mesure de supplanter le régime représentatif. Le principal intérêt des réseaux sociaux réside alors dans le lien qu'il permet de créer entre les représentants de la Nation et ceux au nom desquels ils exercent la souveraineté au Parlement, en dehors des périodes électorales. Cela offre au citoyen un nouveau canal d'expression et de surveillance en mesure de restaurer la confiance politique. Il faut toutefois veiller à ne pas faire des promesses utopiques au risque de distendre encore le lien entre les gouvernants et les citoyens et de mettre en danger la démocratie. 


\title{
LES EffETS DES RÉSEAUX SOCIAUX DANS LES CAMPAGNES ÉLECTORALES AMÉRICAINES
}

\author{
Idris FASSASSI ${ }^{1}$
}

«Sans Twitter, je ne serais probablement pas là. J'ai près de cent millions d'abonnés sur Facebook, Twitter et Instagram. F’ai mon propre média. fe n'ai pas besoin de m'en remettre aux faux médias»². Donald Trump

L'usage des moyens de communication revêt une dimension stratégique fondamentale dans toute tentative de conquête du pouvoir. L'élection présidentielle américaine en 2016 l'a illustré de manière éclatante. En 1800, déjà, la victoire de Thomas Jefferson s'était appuyée sur sa campagne offensive dans la presse contre son adversaire John Adams. Au cours des années 1920, la radio est rapidement devenue un instrument essentiel des campagnes et a profondément modifié la manière dont celles-ci étaient menées. Quelques années plus tard, le Président Franklin Delano Roosevelt s'est d'ailleurs servi avec brio de ses messages radiophoniques pour s'installer au cœur des foyers américains ${ }^{3}$. En 1960, le premier débat présidentiel télévisé de l'histoire, opposant John Fitzgerald Kennedy et Richard Nixon, a marqué un nouveau tournant ${ }^{4}$. La maîtrise de l'outil télévisuel dont a fait preuve le candidat démocrate au cours de sa campagne, et en particulier pendant ce débat, contraste avec l'impréparation et le peu d'intérêt de son adversaire ${ }^{5}$.

Les transformations produites par la révolution numérique constituent les dernières évolutions en date, telles qu'illustrées dans des registres différents par la campagne de Barack Obama en 2008 et celle de Donald Trump en 2016. La première a en effet été qualifiée de "première élection du $\mathrm{xxI}^{\mathrm{e}}$ siècle» en raison notamment de la stratégie de communication novatrice mise en place par le

\footnotetext{
Professeur à l'Université de Picardie Jules Verne, LL.M. Harvard Law School.

Propos de Donald Trump, interview à Fox News, 15 mars 2017.

Voir D. Craig, Fireside Politics. Radio and Political Culture in the United States. 1920-1940, John Hopkins University Press, Baltimore, 2000,

4 M.-A. Watson, «The Kennedy-Nixon debates: The Launch of Television's Transformation of U.S. Politics and Popular Culture », in A. Hoberek, The Cambridge Companion to fohn F. Kennedy, Cambridge University Press, Cambridge, 2015, p. 45 et s.

5 Parmi les facteurs mis en avant pour expliquer la victoire de John Kennedy lors du scrutin final, celui tiré de la bataille de l'image télévisuelle est ainsi fréquemment avancé.
} $362 \mathrm{p}$. 
candidat démocrate ${ }^{6}$. Comme pour John Kennedy, la modernité revendiquée par le candidat s'illustrait dans la maîtrise du nouveau support médiatique. L'élection de 2016 fut indéniablement marquée par les tweets de Donald Trump tout au long de la campagne, comme les propos placés en tête de cet article le rappellent. Sa victoire, qui a déjoué les pronostics de la plupart des observateurs, s'est ainsi appuyée sur une stratégie savamment orchestrée de communication sur les réseaux sociaux, mettant en avant un candidat «hors système» et en prise directe avec les citoyens.

La question des effets produits par les nouvelles technologies de l'information et de la communication sur la démocratie fait l'objet d'études de plus en plus nombreuses ${ }^{7}$, qui présentent néanmoins la particularité d'aboutir à des conclusions souvent opposées. De part et d'autre de l'Atlantique, les auteurs sont en effet divisés sur la portée des changements opérés. Certains mettent en exergue la construction d'un espace public élargi, caractérisé par une culture du dialogue, un engagement politique accru, en particulier chez les jeunes, permettant un approfondissement de la démocratie, ou encore le passage des «médias de masse » aux «médias des masses » ${ }^{8}$. D’autres soulignent, à l'inverse, le danger pour les libertés, l'illusion du renforcement de la participation politique, la réalité d'un cloisonnement de l'individu enfermé dans des «bulles cognitives», le danger de la désinformation et la mise en exergue d'un «agir stratégique» plus que d'un «agir communicationnel»?

Il peut sembler réducteur de vouloir cantonner l'influence ou les effets d'internet à un aspect unique, qualifié de positif ou de négatif. Cela traduit une forme d'essentialisation, peu adaptée pour un outil technologique profondément ambivalent qui devient, pour partie, ce qu'en font ses utilisateurs, et qui est déterminé, dans une large mesure, par ce qu'ont prévu ses concepteurs. En se référant à l'article fondamental du constitutionnaliste et spécialiste du cyberespace, Lawrence Lessig - «Code Is $L a w »^{10}$-, il convient en effet de rappeler que l'architecture de ces outils détermine leur nature. Et parce que cette architecture - le code - évolue et demeure en tout état de cause malléable ${ }^{11}$, la portée de ces outils peut difficilement s'appréhender de manière figée, en étant réduite à une caractéristique unique.

\footnotetext{
A. NAgourney, «The '08 Campaign: Sea Change for Politics as We Know It», The New York Times, 3 novembre 2008 , p. A1.

Voir à titre d'illustration P. SEgur, S. PIERÉ-Frey, L'internet et la démocratie numérique, Presses Universitaires de Perpignan, Perpignan, 2016, 219 p. ; H. Oberdorff, La démocratie à l'épreuve du numérique, Presses Universitaire de Grenoble, Grenoble, 2010, 202 p. ; A. DAHMANI, J. Do-Nascimento, J.-M. Ledjou, J.-J. Gabas (dir.), La démocratie à l'épreuve de la société numérique, Karthala, Paris, 2007,375 p. ; E. Boulot (dir.), Politique, démocratie et culture aux États-Unis à l'ère du numérique, L'Harmattan, Paris, 2011, 192 p ; D. CARDON, La Démocratie Internet. Promesses et limites, Seuil, Paris, 2010, 112 p. ; M. MArgolsis, D. Resnick, Politics as Usual. The Cyberspace "Revolution”, Sage, Londres, 2000, 256 p. ; C. Sunstein, Republic. Com 2.0, Princeton University Press, Princeton, 2007, 272 p.; K. Mossberger, C. TolberT, «How Politics Online Is Changing Electoral Participation », in J. LeIghley (dir.), Oxford Handbook of American Elections and Political Behavior, Oxford, Oxford University Press, 2010, p. 200-215 ; J. Armstrong, M. Zuniga, Crashing the Gate: Netroots, Grassroots, and the Rise of People-Powered Politics, Chelsea Green Publishing, White River Junction, 2006, 208 p.

8 Voir P. DALgren (dir.), Young Citizens and New Media: Learning for Democratic Participation, Routledge, New York, 2007, 276 p. ; D. CARDON, La Démocratie Internet. Promesses et limites, op. cit.

9 Voir M. Hindman, The Myth of Digital Democracy, Princeton University Press, Princeton 2008, 200 p. ; C. Sunstein, \#Republic. Divided Democracy in the Age of Social Media, Princeton University Press, Princeton, 2017, 328 p. ; M. G. SuRAuD, «Le débat électronique. Entre agir communicationnel et stratégie militante», in A. Dahmani, J. Do-Nascimento, J.-M. Ledjou, J.-J. Gabas (dir.), La démocratie à l'épreuve de la société numérique, op. cit., p. 179-192.

10 L. Lessig, «Code Is Law », Harvard Magazine, $1^{\mathrm{er}}$ janvier 2000, accessible en ligne ; voir également du même auteur, Code 2.0, Basic Books, New York, 2006, 410 p.

11 «But no thought is more dangerous to the future of liberty in cyberspace than this faith in freedom guaranteed by the code. For the code is not fixed. The architecture of cyberspace is not given. Unregulability is a function of code, but the code can change », L. LESsIG, «Code Is Law », art. cit.
} 
La présente contribution vise donc à analyser les effets de la révolution numérique et plus particulièrement des réseaux sociaux - entendus comme des plateformes numériques facilitant l'échange d'informations entre individus ${ }^{12}$ - dans la campagne électorale, en s'appuyant sur les enseignements des dernières élections présidentielles américaines. Il y a un intérêt fort pour la situation française dans la mesure où l'usage des réseaux sociaux se développe de façon rapide dans l'hexagone et parce que, dans ces matières comme dans bien d'autres, on constate souvent une migration des phénomènes et des stratégies, de sorte que l'on ne peut ignorer les enseignements de l'expérience américaine ${ }^{13}$.

L'importance des réseaux sociaux dans la campagne américaine tient tout d'abord au poids de ces réseaux dans la société elle-même ${ }^{14}$. On compte ainsi deux cents millions d'utilisateurs de Facebook, soit soixante-huit pour cent de la population totale, et soixante-seize millions utilisateurs de Twitter, soit vingt pour cent de la population ${ }^{15}$. Non seulement ces réseaux sociaux sont très largement utilisés, mais ils sont également le moyen d'accès privilégié à l'information. Soixante-deux pour cent de la population américaine se servent en effet des réseaux sociaux comme source d'accès à l'information ${ }^{16}$.

Les juges de la Cour suprême ont d'ailleurs récemment souligné l'importance de ces réseaux en tant que support de la communication politique. Lors des plaidoiries dans l'affaire Packingham ${ }^{17}$, portant sur la constitutionnalité d'une loi interdisant à certains individus de communiquer sur les réseaux sociaux, la juge Kagan a ainsi affirmé que ces réseaux sont aujourd'hui un «moyen crucial de la communication politique », et le juge Kennedy a fait un parallèle avec le paradigme du forum public $^{18}$. Comme l'a souligné Elena Kagan, tous les membres du Congrès, tous les gouverneurs et, de manière plus générale, la quasi-totalité des candidats à un poste politique disposent d'un compte Twitter ou Facebook. Le poids des réseaux sociaux se mesure également à l'aune des sommes investies par les candidats pour faire campagne sur ce média, soit près de cinq cents millions de dollars pour la dernière campagne présidentielle ${ }^{19}$.

Au-delà de l'aspect quantificatif et financier, se pose la question des effets qualitatifs et des transformations produites par l'utilisation des réseaux sociaux dans la campagne. La question est

12 Parmi les réseaux les plus populaires, on peut ainsi citer Facebook, Twitter, Instagram, Snapchat ou Youtube. Nous nous focaliserons en particulier sur Facebook et Twitter, qui sont aujourd'hui les réseaux sociaux les plus utilisés s'agissant de la discussion politique. En dépit des différences dans la structure même de ces réseaux - Facebook étant ainsi un réseau plus «personnel» - des études soulignent néanmoins que le niveau de contenu politique est équivalent sur chacun de ces réseaux. Voir M. DugGan, A. Smith, «The Political Environment on Social Media », 25 octobre 2016, accessible sur le site du PewResearch Center.

13 Lors de la campagne présidentielle de 2012, l'équipe de campagne de François Hollande avait ainsi sollicité les conseils du responsable de la campagne sur les réseaux sociaux de Barack Obama. Voir infra.

14 Rappelons que les réseaux sociaux les plus populaires dont il est question ici, Facebook et Twitter au premier chef, ont été créés aux États-Unis.

15 PewResearch Center, «Social Media Update 2016 », 11 novembre 2016, accessible sur le site du PewResearch Center.

16 PewResearch Center, «News Use Across Social Media Platforms 2016 », 26 mai 2016, accessible sur le site du PewResearch Center.

17 Packingham v. North Carolina, 137 S. Ct. 1730 (2017). L'affaire concernait une loi interdisant aux individus inscrits sur le registre des délinquants sexuels d'accéder à certains sites tels que Facebook ou Youtube, qui permettent l'échange d'informations et sont ouverts aux mineurs. La loi a été invalidée sur le fondement du Premier amendement protégeant la liberté d'expression.

18 Voir la retranscription des plaidoiries, p. 28, accessible sur le site de la Cour suprême. Sur le lien avec le forum public, voir infra.

19 R. Kay GreEn, «The Game Changer: Social Media and the 2016 Presidential Election », The Huffington Post, 16 novembre 2015, accessible en ligne. 
délicate pour trois raisons. La première tient à la profonde ambivalence en la matière, dans la mesure où la plupart des caractéristiques des réseaux sociaux peuvent s'envisager comme une opportunité ou une tare, comme des vertus ou des vices. La deuxième tient à la complexité de l'objet même de la recherche, liée à la multiplication des niveaux d'analyse. Il faudrait en effet envisager ces effets dans les relations entre les citoyens eux-mêmes, entre les citoyens et les candidats - ce qui pourrait s'envisager du citoyen vers le candidat et du candidat vers le citoyen - et, enfin, entre les candidats eux-mêmes. Le troisième facteur de complexité a trait à la particularité de la campagne électorale elle-même, «moment politique » par excellence. La campagne est en effet à la fois le moment de la mobilisation et de l'action, mais aussi celui de la conviction, de la persuasion et, in fine, de la délibération. Il y a ainsi dans la campagne électorale elle-même une confrontation de deux logiques, et un rapport au temps - temps court et temps long -, voire même à la rationalité, différents.

C'est sous le prisme de cette tension que peuvent en définitive s'appréhender les différents effets des réseaux sociaux dans la campagne, tels que révélés lors des dernières élections américaines. Si les réseaux sociaux sont en effet un catalyseur de la mobilisation politique (I), ils constituent également, dans le même temps, un obstacle à la délibération politique (II).

\section{Les réseaux sociaux en tant que catalyseur de la mobilisation et de l'action politiques}

Il s'agit là de l'effet premier de l'usage des réseaux sociaux en ce qu'ils favorisent, par leur nature même, la mise en action et la réactivité. Ce rôle de catalyseur de l'action politique peut s'envisager de deux manières. S'agissant des citoyens, il permet la mise en œuvre d'une nouvelle forme d'engagement et de participation, auxquels on renvoie parfois à travers l'expression « démocratie connectée »(A). S’agissant des candidats, l'usage des réseaux sociaux est une arme redoutable permettant notamment d'organiser la campagne, d'atteindre par des messages spécifiques des couches particulières de l'électorat, de contourner des obstacles, voire même de renverser des structures établies (B).

\section{A. Un vecteur de l'engagement politique pour les citoyens}

Les effets des réseaux sociaux s'inscrivent dans la lignée des évolutions engendrées par internet s'agissant de la participation politique. Ils tiennent à un accroissement de l'intérêt et de l'engagement politique, en particulier chez les jeunes, à l'établissement d'un nouveau rapport entre les citoyens entre eux, et même entre les citoyens et les candidats est-il parfois soutenu. Les maîtres mots en l'espèce seraient donc l'horizontalité et le dialogue. Les réseaux sociaux permettraient en effet d'aplanir, de niveler les différences et obstacles existant dans le monde « réel ». À titre d'exemple, des activistes, précurseurs de la discussion politique en ligne aux États-Unis, soulignent que ce nouvel espace de l'activisme permet de s'affranchir des contraintes de la politique traditionnelle, qu'il s'agisse de l'argent, des directives de l'establishment des partis ou des médias ${ }^{20}$. Cette nouvelle configuration de l'espace public, par l'aplanissement produit, permettrait en retour un approfondissement du

20 J. Armstrong, M. Moulitsas Zuniga, Crashing the Gate: Netroots, Grassroots, and the Rise of People-Powered Politics, op. cit., p. XV. 
dialogue, à travers le rehaussement de la participation citoyenne. Les citoyens seraient ainsi ramenés au cœur de la discussion politique. L'emploi du conditionnel indique déjà les réserves que l'on peut émettre à l'encontre de cette thèse, sans doute idéaliste, mais qui a néanmoins trouvé certaines concrétisations dès les campagnes de 2004 et de 2008.

Il convient d'ailleurs de porter une attention particulière à la campagne électorale de 2004, dans la mesure où elle illustre les nouvelles potentialités permises par la révolution numérique à travers notamment le phénomène des netroots. Les netroots renvoient à l'origine aux blogs politiques créés à la fin des années 1990 et au début des années 2000 aux États-Unis. Le terme, popularisé par l'activiste Jerome Armstrong en $2002^{21}$, est formé à partir de la contraction des termes «internet» et «grassroots». Il traduit ainsi directement l'idée d'une exploitation de l'outil technologique pour la fédération d'un groupe, à partir de sa base. Ces netroots se sont dans un premier temps développés dans le camp progressiste et les activistes qui les organisèrent avaient pour ambition de se « réapproprier » le parti démocrate ${ }^{22}$. Les netroots progressistes ont dominé la Toile au milieu des années 2000, attirant plus de lecteurs et contributeurs que les netroots conservateurs qui, dans une logique de différenciation, se sont d'ailleurs regroupés sous la bannière des rightroots. Cette domination a pu être justifiée par un argument structurel et idéologique selon lequel le conservatisme serait intrinsèquement incompatible avec la structure ascendante (bottom-up) d'internet ${ }^{23}$. L'argument n'emporte pas la conviction et a d'ailleurs été infirmé par le succès des blogs et des sites conservateurs sous la Présidence Obama et durant la campagne de 2016. Le succès des blogs progressistes au milieu des années 2000, et celui des blogs conservateurs au cours des années 2010, s'expliquent davantage par un facteur conjoncturel, lié au fait que ces mouvements et partis n'étaient pas au pouvoir durant ces période ${ }^{24}$. Le fait d'être dans l'opposition crée ainsi une dynamique, un effet catalyseur, qui luimême pousse à l'exploitation innovante de nouvelles stratégies. Ce recours accru du challenger aux opportunités permises par l'outil numérique se retrouve d'ailleurs en 2008, dans la campagne de Barack Obama face à celle d'Hillary Clinton, et en 2016, dans la campagne de Bernie Sanders face à celle d'Hillary Clinton, ainsi que dans celle de Donald Trump face aux candidats républicains.

En 2004, lors des primaires démocrates, Howard Dean, candidat méconnu du grand public, s'appuya sur les netroots pour mener une campagne qui demeure une référence en matière de mobilisation des jeunes électeurs et d'exploitation de la Toile ${ }^{25}$. Elle préfigure ainsi celle que mena Barack Obama en 2008.

Les aspects fondamentaux des effets des netroots hier et des réseaux sociaux aujourd'hui pour les individus sont triples. En premier lieu, ces outils servent de moyens d'information. C'est en effet

21 J. Armstrong, «Netroots for Dean in $2004 »$, MyDD, 18 décembre 2002, accessible en ligne.

22 Voir V. PiQueT, «L'impact des netroots sur le débat politique et la démocratie américaine : à la fois positif et pernicieux » in E. Boulot (dir.), Politique, démocratie et culture aux États-Unis à l'ère du numérique, op cit., p. 117-135.

23 A. Godet, «The "Other Netroots" : A Study of Online Conservative Activism from 1998 to 2010 », in E. Boulot (dir.), Politique, démocratie et culture aux États-Unis à l'ère du numérique, op cit., p. 28.

$24 \quad$ Ibid., p. 32

25 Voir J. TRIPpI, The Revolution Will Not Be Televised: Democracy, the Internet, and the Overthrow of Everything, HarperCollins publisher, New York, 2004, 272 p. Voir infra. 
à travers Facebook et Twitter que la plupart des Américains s'informent politiquement, ce qui a une influence déterminante au cours de la campagne ${ }^{26}$. Les activistes et groupes investis dans une campagne peuvent utiliser ces réseaux pour produire et diffuser une information alternative, différente de celle véhiculée par le parti, ou les autres partis, en mettant l'accent sur tel ou tel enjeu de la campagne éventuellement négligé. En deuxième lieu, ces outils permettent aux individus de contribuer et de devenir chacun à son niveau, chacun dans son réseau si l'on ose dire, un acteur de la campagne. Il n'est pas donné à tout le monde de pouvoir exprimer son point de vue dans un éditorial publié dans le New York Times, le Washington Post ou sur les ondes, mais les réseaux sociaux permettent précisément à chacun de s'exprimer et de débattre, ou de mobiliser d'autres citoyens.

En troisième lieu, les réseaux sociaux permettent précisément une mobilisation accrue. Cela s'est vérifié dans la campagne de Howard Dean en 2004 et de Barack Obama ultérieurement s'agissant notamment des jeunes. Cette mobilisation n'est pas cantonnée au cyberespace. Le succès d'une campagne réussie tient d'ailleurs à la capacité d'établir des ponts entre l'univers numérique et le monde réel, pour transformer les soutiens en votes le jour de l'élection. À cet égard, la campagne de Barack Obama a illustré les nouvelles potentialités, à travers des opérations de démarchage et de porte-à-porte visant à inciter les électeurs à aller voter le jour de l'élection, qui s'appuyaient sur des informations recueillies sur les réseaux sociaux. Les utilisateurs du réseau social mybarackobama. com recevaient ainsi des informations sur des électeurs indécis se situant à proximité de leur domicile, avec des indications sur le discours à tenir pour les convaincre. La mobilisation et la participation du plus grand nombre étaient ainsi obtenues à travers la contribution des citoyens eux-mêmes, inclus dans la campagne.

Si l'effet catalyseur des réseaux sociaux sur la mobilisation politique en période de campagne électorale est difficilement contestable, il convient d'apporter une précision importante s'agissant de la thèse selon laquelle les réseaux sociaux permettraient la mise en place d'un dialogue entre le candidat et les citoyens. Le fait pour un électeur de suivre un candidat politique sur Twitter ou de le soutenir sur Facebook traduit-il réellement un rapprochement entre ces deux acteurs? À titre d'exemple, l'analyse des comptes Twitter de Barack Obama et de Mitt Romney en 2012, ou de Donald Trump en 2016, révèle que les candidats échangent en définitive très peu avec les citoyens. On ne trouve pas réellement trace de dialogue, et les candidats ne retweetent que rarement des messages de citoyens ordinaires ${ }^{27}$. En 2012, Barack Obama avait lancé une grande opération sur le site Reddit intitulée «Demandez-moi ce que vous voulez » dans laquelle il s'engageait à répondre lui-même aux questions posées par les internautes. Il n'a répondu finalement qu'à dix questions ${ }^{28}$, écartant d'ailleurs certaines questions sensibles. Si les réseaux sociaux favorisent la communication et l'échange

\footnotetext{
26 Voir supra note 14.

27 Une étude menée en juin 2012 sur les messages de Barack Obama et Mitt Romney sur leur compte Twitter révèle que seulement trois pourcent des messages du premier étaient des retweets de messages de citoyens ordinaires, tandis que le second n'a retweeté qu'un seul message au cours de la période, un message de son fils. Voir Pew Research Center, «How the Presidential Candidates Use the Web and Social Media », 15 août 2012, accessible à l'adresse suivante: www.journalism.org/2012/08/15/how-presidential-candidates-use-web-and-social-media/. 28 Les questions et réponses sont accessibles à l'adresse suivante : www.reddit.com/r/IAmA/comments/z1c9z/i am barack obama president of the united states/.
} 
entre les citoyens eux-mêmes ${ }^{29}$, il n'est donc pas établi qu'ils permettent un véritable dialogue entre les citoyens et le candidat.

On constate en l'espèce une asymétrie et la tendance unidirectionnelle de la communication sur ces réseaux sociaux, du candidat vers le citoyen, ce qui s'inscrit de manière plus large dans le cadre des opportunités offertes par les réseaux sociaux aux candidats.

\section{B. Une arme de campagne redoutable pour les candidats}

Il s'agit de la dimension qui vient immédiatement à l'esprit suite à l'élection de 2016, dans la mesure où le Président Trump lui-même affirme qu'il n'aurait probablement pas été élu sans Twitter $^{30}$. Dans des styles différents, Barack Obama en 2008 et Donald Trump en 2016 ont ainsi su se servir de ce nouvel outil.

S'agissant du premier, sa campagne de 2008 a repris, développé et perfectionné les axes de celle menée par Howard Dean en 2004. Sur ce point, Barack Obama a eu la clairvoyance de mesurer très tôt la portée des réseaux sociaux et d'investir en la matière ${ }^{31}$. Dès 2007, il a ainsi engagé Chris Hughes, un des cofondateurs de Facebook, qui a mis en place sa stratégie de communication en ligne. Celle-ci s'organisait autour d'un site, barackobama.com, d'un réseau social autonome, mybarackobama.com, qui permettait aux internautes de se connecter entre eux, et d'un usage particulièrement efficace des réseaux sociaux classiques tels que Facebook, Twitter ou encore YouTube. Quelques données statistiques permettent de mesurer l'impact de cette stratégie. Plus de deux millions de profils ont ainsi été créés sur mybarackobama.com, plus de deux cent mille évènements ont été organisés, plus de quatre cent mille billets ont été rédigés, et plus de trente-cinq mille groupes de volontaires ont été créés ${ }^{32}$.

Cette stratégie active du candidat démocrate lui a permis d'établir des écarts significatifs avec ses adversaires en termes de soutien en ligne. Ainsi, lors des primaires démocrates, Barack Obama avait deux cent cinquante mille abonnés sur Facebook, contre seulement trois mille deux cents pour Hillary Clinton. Lors de l'élection générale, le candidat démocrate, avec deux millions d'abonnés sur Facebook, comptabilisait quatre fois plus de soutiens que son adversaire John McCain, et vingt fois plus sur Twitter ${ }^{33}$. En 2012, il comptait également trois fois plus de soutiens sur Facebook que le candidat républicain Mitt Romney, et douze fois plus de soutiens sur Twitter ${ }^{34}$. Lors des primaires républicaines de 2016, Donald Trump a lui aussi largement dominé ses adversaires sur Twitter et Facebook. Il disposait également d'un net avantage lors de l'élection générale face à Hillary Clinton, non seulement concernant ses soutiens sur les réseaux sociaux - deux fois plus par exemple sur

29 Avec la réserve que cette communication accrue n'opère le plus souvent qu'entre des individus relevant du même environnement idéologique. Voir infra.

$30 \quad$ Voir supra note 1.

31 Voir V. ChAng, «Obama and the power of social media and technology», European Business Review, mai-juin 2010, p. 16-21; M. BANWART, M. McKinney (dir.), Communication in the 2008 U.S. Election: Digital Natives Elect a President, Peter Lang, New York, 2011, p. 89-104.

32 J. VArgas, «Obama Raised Half a Billion Online», The Washington Post, 20 novembre 2008, accessible en ligne.

33 S. Dutta, M. Fraser, «Barack Obama and the Facebook Election», accessible à l'adresse suivante: www.usnews.com/opinion/ articles/2008/11/19/barack-obama-and-the-facebook-election.

34 S. Frantzich, «Are We Halfway There Yet?: New Technology and the 2012 Election » in W. Crotty (dir.), Winning the Presidency 2012, Paradigme, New York, 2013, p. 93-94. 
Facebook -, mais aussi plus généralement au regard de son empreinte numérique, c'est-à-dire sa capacité à capter l'attention et à faire relayer, retweeter et partager ses messages ${ }^{35}$.

À l'évidence, une domination sur la Toile et les réseaux sociaux n'est pas en elle-même suffisante pour pouvoir remporter une élection ${ }^{36}$. Elle représente néanmoins un atout considérable lorsque le candidat parvient à transformer ce soutien sur les réseaux en un soutien opérationnel. La campagne de Barack Obama a d'ailleurs été une réussite sur ce point avec l'utilisation combinée des outils les plus modernes et des stratégies les plus anciennes, à savoir l'usage des réseaux sociaux pour recueillir des informations sur les électeurs potentiels, et du porte-à-porte pour ensuite les convaincre de s'inscrire sur les listes électorales et de voter ${ }^{37}$.

Outre cet aspect de mobilisation générale de l'électorat, la dimension offensive de l'usage des réseaux sociaux pour le candidat se décline sous plusieurs aspects.

Elle permet tout d'abord d'atteindre des couches particulières de l'électorat à travers une communication ciblée. Cela a été le cas particulièrement dans le cadre de la campagne de Barack Obama en 2008 s'agissant des jeunes et de certains groupes et communautés. Les pages de soutien Facebook du candidat ont ainsi été déclinées en différentes variantes, avec par exemple, les Veterans for Obama, Women for Obama, etc. Dans le même sens, ses équipes de campagne ont créé des profils spécifiques sur certains réseaux pour atteindre les communautés noire, asiatique ou hispanique ${ }^{38}$. Dans cette optique, les réseaux sociaux sont utilisés pour cibler et capter un électorat particulier, ce qui présente déjà le risque d'un morcellement du discours politique.

En second lieu, les réseaux sociaux sont un outil formidable pour lever des fonds. Howard Dean est le pionnier en la matière ${ }^{39}$. En 2004, au lieu de chercher à recueillir le financement des donateurs traditionnels du parti démocrate, la campagne du candidat se focalisa sur des milliers de petits donateurs, les sympathisants et citoyens ordinaires, contactés sur la Toile, à travers les différents netroots et des sites tels que MoveOn et MeetUp. Si cette stratégie a été innovante, la réalité est que Howard Dean n'avait pas d'autre choix, dans la mesure où il n'était pas soutenu par la direction du parti et ses donateurs traditionnels. On retrouve ici l'idée précédemment évoquée du lien entre nécessité et innovation, qui pousse les challengers à exploiter au maximum les opportunités de la Toile. Howard Dean établit ainsi de nouveaux records côté démocrate, recueillant plus de quinze millions de dollars sur le dernier trimestre de l'année 2003, et plus de cinquante millions de dollars au total, provenant majoritairement de contributions inférieures à 200 dollars $^{40}$. En 2008, l'équipe de campagne de Barack Obama s'inspira et développa cette stratégie, permettant au candidat de récolter la somme

\footnotetext{
35 Pew Research Center, Election 2016: Campaigns as a Direct Source of News, 18 juillet, 2016, p. 15 et s., accessible en ligne; « Clinton vs. Trump: How They Used Twitter », Wall Street fournal, 7 avril 2017, accessible en ligne.

36 L'échec de Howard Dean en 2004 à obtenir l'investiture côté démocrate en est d'ailleurs une illustration.

37 Voir supra.

38 BlackPlanet.com, Asianave.com, Migente.com

39 Voir l'ouvrage rédigé par son directeur de campagne, J. TRIPPI, The Revolution Will Not Be Televised: Democracy, the Internet, and the Overthrow of Everything, op. cit.

40 Institute for Politics Democracy and the Internet, Campaign Finance Institute, Small Donors and Online Giving, A study of Donors to the 2004 Presidential Campaign, p. 3-4, accessible sur le site du Campaign Finance Institute.
} 
record de plus de sept cent quarante millions de dollars. Le candidat démocrate bénéficia ainsi de la force de frappe de la société Blue State Digital, dirigée par des anciens de la campagne de Howard Dean, qui mit en place une stratégie numérique globale alliant big data, communication profilée et collecte de fonds.

La mise en exergue des potentialités d'internet et des réseaux sociaux pour lever des fonds conduit logiquement à s'interroger sur l'état de la réglementation en la matière concernant le financement des campagnes. Il apparaît que porter la campagne sur la Toile permet, dans une large mesure, de s'affranchir des réglementations traditionnelles. En effet, l'espace en ligne est, en quelque sorte, une terre vierge, très faiblement réglementée par la puissance publique. Le principe fondamental, imposé par la Commission électorale fédérale (FEC), est que toute communication placée sur un site en échange d'une somme d'argent tombe sous le coup de la réglementation classique relative aux «communications publiques $»^{41}$. Au-delà de cet aspect minimal, il existe plus de questions que de réponses ${ }^{42}$.

En troisième lieu, l'usage des réseaux sociaux par le candidat peut être un outil offensif permettant d'attaquer des adversaires. En 2015 et 2016, les nombreux tweets de Donald Trump à l'encontre de Jeb Bush, Ted Cruz et Marco Rubio durant les primaires, et à l'encontre d'Hillary Clinton durant l'élection générale sont particulièrement révélateurs. La campagne de Donald Trump illustre ainsi un usage plus agressif des réseaux sociaux, destiné non pas uniquement à organiser, informer ou dynamiser la campagne du candidat, mais à attaquer directement des adversaires, en les forçant parfois à croiser le fer sur ce terrain ${ }^{43}$.

L'élection présidentielle de 2016 a révélé une autre potentialité s'agissant de l'usage des réseaux sociaux par un candidat, à savoir la possibilité de s'en servir pour renverser et transcender des structures établies. C'est d'ailleurs le sens des propos de Donald Trump cités au début de cet article, à savoir l'idée que sans Twitter il n'aurait pu remporter l'élection ${ }^{44}$. Il y a indéniablement une dimension utopique dans la thèse selon laquelle les réseaux sociaux permettent « au petit candidat » de se faire entendre et de résister au «système ». Il faut donc veiller à ne pas succomber à un excès d'idéalisation, en gardant à l'esprit que la révolution numérique sert principalement et le plus souvent des candidats disposant d'atouts importants ${ }^{45}$. En d'autres termes, si Donald Trump était clairement un candidat atypique, il n'était pas pour autant un «petit candidat» au regard de sa fortune ou de ses réseaux. Il a toutefois réussi à se servir de Twitter pour court-circuiter et même s'affranchir des médias classiques. Comme il le dit lui-même, le fait de disposer de son propre média - à travers les réseaux sociaux - et de pouvoir diffuser directement ses messages à un auditoire de plusieurs

\footnotetext{
41 Voir le rappel des principes applicables aux communications en ligne accessible sur le site de la FEC, à l'adresse suivante : www.fec.gov/ pages/brochures/internetcomm.shtml.

42 Il convient de préciser ici que la faible intervention de la puissance publique ne signifie pas que ces espaces sont soustraits à toute réglementation. La nature ayant horreur du vide, il apparaît que les réseaux sociaux eux-mêmes, c'est-à-dire les entreprises Facebook ou Twitter, deviennent en quelque sorte les régulateurs et peuvent décider dans une large mesure quel type de discours est permis. On assiste ainsi à une privatisation problématique de la règlementation applicable au cyberespace. Voir infra.

43 Voir infra.

$44 \quad$ Voir supra note 1.

45 Voir M. Hindman, The Myth of Digital Democracy, op. cit.
} 
dizaines de millions de personnes, lui a permis de s'affranchir des médias traditionnels. Sa campagne est de ce point de vue pionnière, dans la mesure où il a profondément chamboulé les codes de la politique outre-Atlantique et, dans une certaine mesure, réécrit les règles du jeu. On assiste ainsi à une nouvelle forme de story telling, permettant au candidat de reprendre la main sur les médias.

Un même scénario s'est d'ailleurs reproduit à chaque fois que ses déclarations ou actes étaient perçus comme excessifs et que certains observateurs annonçaient prématurément la fin de sa campagne. Donald Trump a alors le plus souvent surenchéri et dicté le tempo médiatique, tweet après tweet. Ce faisant, il a forcé les médias classiques à s'ajuster à son propre rythme et à devenir, en quelque sorte, les commentateurs de son propre compte twitter.

Le renversement à l'œuvre ne s'est pas limité aux médias. La campagne de 2016 côté républicain traduit également l'affaiblissement du parti en tant qu'institution. Les primaires ont en effet conduit à la désignation d'un candidat rejeté par l'establishment du parti, qui a néanmoins pu faire campagne en s'affranchissant largement des structures de ce même parti. Les réseaux sociaux, précisément, lui ont permis de mettre en œuvre sa propre communication et de ne pas être dépendant de celle organisée par le parti, et les règles relatives au financement des campagnes électorales, autorisant les entreprises et donateurs fortunés à dépenser de manière quasiment illimitée, permettent, là encore, de court-circuiter le parti ${ }^{46}$.

Les lignes qui précèdent illustrent en quoi les réseaux sociaux sont une arme pour le candidat pour atteindre certaines couches de l'électorat, organiser sa campagne, lever des fonds, attaquer directement ses adversaires ou encore court-circuiter les médias classiques. Cette arme est néanmoins à double tranchant et peut se retourner contre le candidat. L'hypothèse évidente est celle du tweet impulsif, regretté puis effacé par le candidat, comme ce fut le cas pour Donald Trump et Ted Cruz notamment durant les primaires. La difficulté est que ce tweet réapparaît le plus souvent sur la twittosphère. Les médias ont même rapporté qu'afin d'éviter tout dérapage de dernière minute, l'équipe de campagne de Donald Trump l'aurait privé de son compte Twitter dans les derniers moments de la campagne ${ }^{47}$. Ce versant négatif de l'usage des réseaux sociaux par le candidat lui-même permet de faire le lien avec les obstacles posés par l'usage de ces réseaux à la délibération politique de manière plus générale.

\section{L’usage des réseaux sociaux en tant qu'obstacle à la délibération politique}

Si la campagne électorale est le moment de la mobilisation, pour les citoyens comme pour le candidat, elle est aussi celui, du moins en théorie, du débat d'idées et de la délibération politique. La campagne doit en effet permettre la confrontation réfléchie de programmes et de propositions politiques. À cet égard, on peut toutefois considérer que la communication sur les réseaux sociaux,

46 Qu'il nous soit permis de renvoyer à notre commentaire sous l'arrêt Citizens United v. FEC, 558 U.S. 310 (2010), RFDC, 2011, p. 324 et s.
47 M. Haberman, A. Parker, J. Peters, M. BArbaro, «The Storm Below the Calm: An Inside View of Trump's Last Stand», The New York Times, 7 novembre 2016, p. A1. 
qui repose principalement sur une logique d'instantanéité, de simplification formatée et parfois de désinformation, contribue à l'appauvrissement du débat politique (A). Les derniers enseignements de la campagne de 2016 permettent de pousser l'argument plus loin. L'effet de bulle produit par les réseaux sociaux tenant au fait que les utilisateurs sont confortés dans leurs opinions, par l'exposition à des opinions similaires, et non confrontés à des points de vue opposés, conduit, in fine, à la négation du débat (B).

\section{A. L'appauvrissement du débat}

Le principe actif des réseaux sociaux, et de Twitter en particulier, est la réactivité. Cette dimension, très utile, dans une logique d'action, est plus problématique à l'égard des vertus attendues de la campagne quant à la délibération. On constate ainsi une course effrénée vers l'avant, dans la mesure où le candidat n'existe qu'à travers son dernier tweet. Un compte inactif est un compte qui se déprécie. Le réseau social, et les attentes qu'il produit, structurent donc la communication des candidats et l'oriente dans un sens contraire à la délibération. En d'autres termes, le temps court s'oppose ici au temps long.

La campagne de 2016 a illustré les dérives auxquelles cela peut mener, à travers des échanges brutaux, agressifs, et injurieux de la part des candidats et des citoyens. L'outil offensif évoqué précédemment a donc également été, à bien des égards, offensant. On pense tout d'abord aux différents messages de Donald Trump à l'encontre de journalistes, de personnalités publiques et d'autres candidats. Le New York Times tient à ce sujet une base de données relative aux différentes «personnes, lieux et choses» que Donald Trump a insultés sur Twitter depuis son entrée dans la campagne électorale. Le chiffre est actualisé en fonction des derniers tweets et est actuellement de quatre cent vingt-cinq ${ }^{48}$. On notera, entre autres, les attaques contre Hillary Clinton qualifiée de «stupide», contre la journaliste Megan Kelly - « folle» -, Barack Obama - « incompétent » et «pire Président de l'histoire » - la sénatrice Elisabeth Warren - «Pocahontas», en référence à l'affaire sur ses origines amérindiennes. On se souvient également qu'il avait retweeté une photo de la femme d'un adversaire, Ted Cruz, juxtaposée à celle de sa femme, avec pour légende «l'image parle d'elle-même».

De tels échanges illustrent un abaissement problématique du débat et les messages de Donald Trump par exemple sont loin d'être l'unique illustration. Ils sont symptomatiques d'une tendance plus générale. De nombreuses études concluent en effet que l'hostilité, l'agressivité et l'injure sont des caractéristiques de la communication en ligne, en raison de la désinhibition produite par l'anonymat et la médiation de l'écran ${ }^{49}$. Des études récentes menées pendant la campagne électorale de

48 J. Lee, K. Quealy, «The 425 People, Places and Things Donald Trump Has Insulted on Twitter: A Complete List», accessible sur le site du New York Times.

49 Voir le recensement des travaux en ce sens, in F. Greffet, S. Wojcik, «Parler politique en ligne. Une revue des travaux français et anglosaxons », Réseaux, nº 4, 2008, p. 31 et s; K. Wilcox, A. Stephen, «Are Close Friends the Enemy? Online Social Networks, Self-Esteem, and Self-Control», fournal of Consumer Research, juin 2013, p. 90-103. 
2016 soulignent dans le même sens qu'une majorité d'utilisateurs des réseaux sociaux jugent que la discussion politique y est «particulièrement négative », en raison du ton retenu ${ }^{50}$.

L'appauvrissement du débat s'illustre également à travers la propagation, grâce précisément aux réseaux sociaux, des «fausses informations». Cette question et l'importance supposée de ces campagnes de désinformation sur l'issue du dernier scrutin présidentiel ont d'ailleurs conduit le dirigeant de Facebook, Mark Zuckerberg, à s'expliquer quelques jours après le résultat ${ }^{51}$. Le but était de souligner l'attention accordée par Facebook à la question de l'intégrité de l'information et d'envisager des moyens de la garantir, à travers notamment la possibilité d'un signalement des informations douteuses. On se trouve là face à l'illustration d'une des ambivalences de l'usage des réseaux sociaux. D'un côté, ils accroissent la participation en permettant à un plus grand nombre d'individus de rejoindre le débat, mais ils conduisent également à brouiller les lignes, dans la mesure où le nivellement opéré rend plus délicat tout effort de distinction entre l'information sérieuse et celle qui ne l'est pas. Le numérique favorise ici un rapport distendu à la vérité et l'objectivité. Ce rapport distendu se retrouve d'ailleurs dans certains tweets de Donald Trump, dans lesquels ils relaient des informations qui sont ensuite démenties, ou dans lesquelles ils énoncent des affirmations graves sans apporter de preuves ${ }^{52}$.

On ne peut manquer de noter l'ironie tenant à ce qu'un des bienfaits invoqués des netroots tenait à la forme de vigilance qu'ils permettaient, en plaçant sur le devant de la scène des faits ignorés par les médias classiques ${ }^{53}$. La Toile était alors envisagée comme un instrument de résistance permettant précisément de faire éclore la vérité. Aujourd'hui, la problématique se pose en sens inverse et l'on s'interroge sur les manières de réintroduire une hiérarchie des informations, une forme de verticalité afin de couper le phénomène de propagation horizontale des fausses informations sur les réseaux.

L'appauvrissement du débat politique peut également s'envisager à l'aune de la standardisation du message sur les réseaux sociaux. Sur Twitter par exemple, les candidats doivent se plier à la contrainte des cent quarante caractères, ce qui conduit à des raccourcis dans l'expression, dont on peut toutefois craindre qu'ils traduisent aussi un raccourci de la pensée. On peut également s'interroger sur la mesure dans laquelle les réseaux façonnent la réception par le citoyen du message politique. L'usager est en effet contraint et limité par les formes de réaction prévues par le réseau social lui-même. À travers les «partages», «j'aime» ou « retweets», s'installe une forme de standardisation des comportements, sans doute inadaptée à la réception et la pleine compréhension d'un message politique. On peut craindre en effet que la délibération politique perde sa richesse et sa spécificité, en raison de la superficialité de son appréhension ${ }^{54}$.

\footnotetext{
50 Voir M. Duggan, A. Smith, «The Political Environment on Social Media », art. cit.

51 Voir le billet de Mark Zuckerberg, accessible à l'adresse suivante

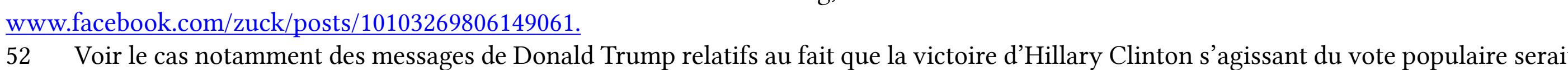
due aux votes frauduleux d'individus en situation irrégulière.

53 Voir V. PIQUeT, «L'impact des netroots sur le débat politique et la démocratie américaine : à la fois positif et pernicieux », art. cit., p. 125127.

54 Pour une approche très critique des changements engendrés par Internet, et notamment des effets négatifs, sur la capacité des individus à penser, voir N. CARr, The Shallows : What the Internet Is Doing to Our Brains, W.W. Norton, New York, 2010, 276 p.
} 
À cet appauvrissement constaté du débat s'ajoute même, en définitive, une certaine négation du débat.

\section{B. La négation du débat}

Les réseaux sociaux permettent de se couper de toutes opinions diverses, d'opérer en circuit fermé et donc de rejeter le pluralisme sans lequel, pour reprendre la célèbre formule de la Cour européenne des droits de l'homme, «il n'est pas de société démocratique ${ }^{55}$. Dans le monde réel, le monde de tous les jours, il est difficile de ne pas voir ce qui se donne à voir. Il est difficile en effet de ne pas être confronté à des opinions diverses. Aux États-Unis, ceci a une implication particulière pour ce qu'on appelle la théorie du «forum public » en matière de liberté d'expression ${ }^{56}$. Dans ces forums publics, dont la rue, le trottoir ou le parc sont des exemples topiques, il ne peut y avoir de règlementation fondée sur le contenu de l'expression. Toutes les opinions, à quelques rares exceptions près, doivent pouvoir être défendues. Un des fondements philosophiques de cette conception maximaliste de la liberté d'expression qui prévaut aux États-Unis est d'ailleurs la métaphore du marché des idées politiques, mise en exergue par le juge Holmes dans son opinion dissidente dans l'affaire $\mathrm{Abrams}^{57}$. Cette approche puise dans la pensée des philosophes John Milton et John Stuart Mill et considère que la vérité n'apparaît pleinement que dans sa confrontation avec l'erreur. Dès lors, la collision des opinions contraires est le meilleur moyen de faire triompher la vérité. John Stuart Mill affirmait ainsi «que l'on ne saurait surestimer l'intérêt qu'il y a à mettre des êtres humains en contact avec des personnes qui ne leur ressemblent pas et dont les modes d'action et de pensée différent de ceux auxquels ils sont habitués $»^{58}$. Il y voyait là une des sources du progrès.

Dès lors qu'aujourd'hui, les parcs et les rues ont été pour partie supplantés par le cyberespace en tant qu'espace de formation de l'opinion ${ }^{59}$, la question est de savoir comment assurer la pluralité et la diversité des interactions dans un univers où l'utilisateur peut se façonner son propre réseau cloisonné. En d'autres termes, où sont les équivalents des trottoirs et des parcs publics dans le cyberespace ${ }^{60}$ ?

\footnotetext{
55 «[la liberté d'expression] vaut non seulement pour les “informations” ou "idées" accueillies avec faveur ou considérées comme inoffensives ou indifférentes, mais aussi pour celles qui heurtent, choquent ou inquiètent l'État ou une fraction quelconque de la population. Ainsi le veulent le pluralisme, la tolérance et l'esprit d'ouverture sans lesquels il n'est pas de "société démocratique" », CEDH, Handyside c. Royaume-Uni, 7 décembre 1976, $\mathrm{n}^{\circ}$ 5493/72.

56 Voir E. Zoller, «La Cour suprême et la liberté d'expression», in E. Zoller (dir.), La liberté d'expression aux États-Unis et en Europe, Dalloz, Paris, 2008, p. 259 et s. ; R. Post, «Between Governance and Management : The History and Theory of the Public Forum», UCLA Law Review, 1987, p. 1713-1836.

57 «Le meilleur test pour la vérité est la capacité de la pensée à se faire accepter dans la compétition du marché », Abrams v. United States, 250 U.S. 616,630 (1919).

58 J. Stuart Mill, Principes de l'économie politique, Tome II, Guillemin et Compagnie, Paris, 1861, p. 124, accessible sur le site de la bibliothèque numérique archive.org.

59 C'est d'ailleurs ce que reconnaît le juge Anthony Kennedy: « Minds are not changed in streets and parks as they once were. To an increasing degree, the more significant interchanges of ideas and shaping of public consciousness occur in mass and electronic media », Denver Area Educ. Telecomm. Consortium, Inc. v. FCC, 518 U.S. 727, 803 (1996).

60 Cf. N. ZATz, «Sidewalks in Cyberspace: Making Space for Public Forums in the Public Environment», Harvard fournal of Law and Technology, vol. 12, 1998, p. 149-240.
} 
Ce problème a été mis en exergue depuis longtemps, notamment par le juriste Cass Sunstein à Harvard qui, dans une série d'ouvrages ${ }^{61}$, a dénoncé le danger des filtres et des «cocons cognitifs» qui fragmentent la société et réduisent la masse des «expériences partagées » par les membres de la société, condition pourtant essentielle à son bon fonctionnement. Le danger vient donc du fait que, sur les réseaux sociaux, il est possible de créer sa propre réalité, et de constituer ainsi son propre forum privé, un réseau que l'on pourrait sans doute qualifier d'idiot, en se référant à l'origine étymo-

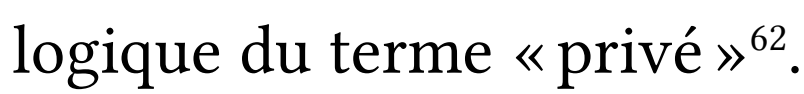

La structure même des réseaux sociaux conduit à n'offrir à l'utilisateur qu'une vision partielle du champ des possibles, celle qu'il choisit en s'entourant de personnes qui partagent ses opinions politiques, et celle que le réseau choisit pour lui à travers l'algorithme utilisé pour la présentation des informations. Il y a là une tension entre l'ouverture supposée d'internet et des réseaux sociaux, et la réalité de leur fonctionnement, qui repose pour partie sur un enfermement et une cristallisation de la pensée. Ceci risque de produire un aveuglement et une radicalisation.

Au cours de la campagne de 2016, certains supporters de Donald Trump se disaient convaincus de sa victoire et affirmaient que s'il perdait, cela serait la preuve que l'élection a été achetée. Ils en voulaient pour preuve le fait qu'ils voyaient une majorité de messages en sa faveur sur les réseaux sociaux ${ }^{63}$. On ne saurait trouver meilleure preuve d'un raisonnement circulaire, biaisé par le prisme déformant des réseaux sociaux. Dans le même ordre d'idées, mais en sens inverse, l'incapacité des grands médias à prévoir la victoire de Donald Trump a été également justifiée par les effets aveuglants des réseaux sociaux ${ }^{64}$.

S'agissant des écueils posés par l'usage massif des réseaux sociaux, un dernier élément doit être évoqué, relatif au pouvoir de ces réseaux. Ils peuvent, nous l'avons vu, réglementer le contenu des discussions en ligne, en imposant des règles qui, si elles étaient adoptées par les pouvoirs publics, seraient probablement jugées inconstitutionnelles aux États-Unis ${ }^{65}$. Ils décident également de ce que voit l'utilisateur, en fonction de l'algorithme utilisé. Certains auteurs se sont interrogés sur la capacité de ces réseaux à influencer directement une élection, par exemple, en incitant une partie de la population à voter. Une expérience menée en 2010 a d'ailleurs mis en lumière les effets directs de Facebook sur le processus électoral, en encourageant, par des messages adressés à une partie de la population, dans des zones spécifiques, la participation ${ }^{66}$. Ces résultats, et les hypothèses qu'ils

\footnotetext{
61 C. Sunstein, \#Republic: Divided Democracy in the Age of Social media, Princeton University Press, Princeton, 2017,328 p.; Republic. Com 2.0, Princeton University Press, Princeton, 2007, 272 p.; Republic. Com, Princeton University Press, Princeton, 2001, 224 p. Voir également E. PARISER, The Filter Bubble: What The Internet Is Hiding From You, Penguin Press, New York, 2011, $294 \mathrm{p}$.

62 Idiotes renvoie ainsi au particulier, à ce qui se prive d'une partie du monde. Sur le public et le privé, voir notamment J.-A. MAZÈres, «Public et privé dans l'œuvre d'Hannah Arendt : de l'opposition des termes aux termes de l'opposition », RDP, 2005 , p. 1047 et s.

63 A. Parker, N. Corasaniti, «Some Donald Trump Voters Warn of Revolution if Hillary Clinton Wins », The New York Times, 27 octobre 2016, accessible en ligne.

64 Voir J. Wortham, «Is Social Media Disconnecting Us From the Big Picture? », The New York Times Magazine, 22 novembre 2016 , accessible en ligne.

65 Voir supra note 42

66 Voir J. ZitTrAin, «Engineering an Election: Digital Gerrymandering Poses a Threat to Democracy », Harvard Law Review Forum, vol. 127, 2014, p. 335-341.
} 
laissent entrevoir, renforcent la nécessité d'imposer des règles de transparence sur le fonctionnement de ces réseaux.

Malgré les indéniables défis posés par l'usage des réseaux sociaux, en particulier pour la qualité de la délibération démocratique, il convient d'éviter de porter un jugement trop sévère à leur encontre. Il est en effet très délicat d'opérer un bilan net de leur apport, et on ne saurait négliger les opportunités qu'ils représentent, en termes notamment de mobilisation et de participation des citoyens à la discussion politique. Il convient également de contextualiser le discours actuel, plutôt critique à l'égard des réseaux sociaux, en rappelant les appréciations toutes autres qui étaient portées sur ces outils après les élections de 2008 ou 2012 outre-Atlantique. Après les victoires de Barack Obama, les réseaux sociaux étaient en effet plutôt perçus comme des instruments vertueux et émancipateurs. Après les élections de 2016, le ton est beaucoup plus sévère. Sans idéaliser ni diaboliser ces réseaux, il est possible d'appréhender leurs effets de manière plus mesurée, en considérant qu'ils reflètent plus qu'ils ne créent la polarisation et les tensions de la société américaine aujourd'hui. Ces réseaux ancrent, amplifient sans doute, ces travers, mais ils ne les créent pas par eux-mêmes. Les réseaux ne font qu'accentuer les tendances lourdes de l'environnement dans lequel ils opèrent.

En tout état de cause, les solutions à certains des écueils avérés pourraient être apportées par les réseaux eux-mêmes, par la modification de leur code et de leur architecture. On peut ainsi imaginer, à l'instar de Cass Sunstein, que ces réseaux incluent des options permettant d'exposer l'utilisateur à des points de vue opposés et à des informations choisies de manière aléatoire, hors de ses champs d'intérêt ${ }^{67}$. Si techniquement la solution est possible, demeure la question des moyens permettant d'imposer à des entités privées aussi puissantes des réformes qui contribueraient sans doute au bien commun.

67 C. Sunstein, \#Republic: Divided Democracy in the Age of Social Media, op. cit., p. 231 et s. 



\section{Discussion}

Président (Pascal Jan): Peut-être une ou deux observations. La première, et je rebondis sur le titre «la démocratie connectée », à vous entendre tous les trois, même depuis ce matin, c'est plutôt une démocratie instantanée, voire simplifiée. Ce qui n'est pas tout à fait la même chose que connectée. C'est la différence entre les blogs et les réseaux sociaux du type de Facebook et Twitter. C'est intéressant car dans les débats d'aujourd'hui, on se focalise beaucoup sur Twitter, c'est-à-dire un type de réseau très particulier où les caractères sont limités, où on est dans la réaction, dans la dénonciation, dans la critique. On ne parle pas dans les réseaux sociaux de la même façon que dans les blogs. Il y a peut-être un champ à travailler parce que cela ne fait pas appel aux mêmes réflexes de la part des citoyens.

Ma deuxième remarque est que l'on a l'impression, après les communications sur les partis politiques et les parlementaires, mais cela se vérifie avec la dernière campagne présidentielle américaine, que nous ne sommes absolument pas dans la délibération, c'est une certitude, ni dans la discussion collective: il s'agit d'une relation bilatérale, quelque part nous ne sommes absolument pas dans le collectif. En réalité, nous nous apercevons que ce sont des réactions très isolées et très autonomes les unes par rapport aux autres, ce qui pose un problème de cohérence et de synthèse. Avec la loi Lemaire, la loi sur le numérique, nous constatons qu'il y a une multitude de propos très personnels et des lobbies, et que tout cela ne donne pas quelque chose de très cohérent. Il y a une difficulté pour le pouvoir politique, à « digérer » l'information qui est donnée. Il y a le fait de « s'exprimer » et le fait de «digérer» l'information donnée. Il y a un problème pour les pouvoirs politiques qui n'ont pas appris et qui ne sont pas forcément prédisposés à gérer une multitude d'informations.

Je me souviens il y a quelques années à Science Po Bordeaux, nous avions invité Ségolène Royal juste avant l'élection présidentielle de 2007. Elle était favorable à la démocratie participative et était très en avance sur les réseaux sociaux. Elle agissait, au niveau national et au niveau local, déjà énormément de ce point de vue là. Nous avions demandé aux étudiants de faire une enquête sur ce qui avait été annoncé et ce qui avait été réalisé dans ce domaine. C'est-à-dire, autant en termes de démocratie dite participative, que ce soit l'intervention des citoyens sur des forums ou encore dans des consultations publiques, etc. Le résultat est que par rapport à cette participation des citoyens, sous toutes ces formes, il n'y avait aucun suivi, aucune reprise. Oui, la parole était donnée; oui, il y avait des réactions, mais en réalité, il n’y avait aucune concrétisation. C'est sur cela aussi que l'on 
doit s'interroger: n'est-ce pas la manipulation (le terme est sans doute trop fort) d'une opportunité pour valoriser ou légitimer une action plutôt que réellement entamer une nouvelle façon de penser la démocratie?

La démocratie connectée peut représenter une opportunité, mais aussi un danger dès lors que le «contrat» n'est pas aussi clair que cela entre les gouvernants et les gouvernés. Il ne faut pas oublier une chose c'est que s'il y a une crise, en France et dans d'autres pays tels que les États-Unis, c'est qu'il y a une distanciation entre ce qui est dit, ce qui est promis, et la réalité ou en tout cas le ressenti des citoyens.

Quelle serait ou quelle sera la réaction des citoyens si cette démocratie connectée venait à se développer et si, en réalité, il n'y avait aucun suivi, aucun résultat? Je ne suis pas sûr que cela favorise véritablement les parlementaires et les élus. Je pense que la réaction pourrait être assez violente de ce point de vue là, parce qu'aujourd'hui il y a une sorte de défouloir, de communication, de lien. Ce lien n'existera plus quand bien même il est aujourd'hui purement virtuel.

Public (Jean Gicquel): Juste une observation sur votre formule finale : réseaux sociaux et juge, à titre anecdotique, il y a deux ans de cela, le Conseil supérieur de la magistrature a été amené à se prononcer, en matière disciplinaire, sur le comportement de deux juges. Ces derniers, au lieu de juger, «twittaient». Cela se passait devant ce qui est considéré par tous les magistrats comme la vitrine de la Justice: la Cour d'assises. L'affaire mettait en cause un magistrat du siège et un magistrat du parquet. Les messages sont tombés dans le domaine public, ils ont été poursuivis par le garde des Sceaux et ont été sanctionnés et déplacés d'office. Pire des hypothèses pour un magistrat du siège, être déplacé d'office c'est manquer au principe d'inamovibilité. Quand on est juge, on doit juger et non pas twitter! (Rires)

Public: Mon intervention fait suite à celle de Monsieur Bardin qui disait que le numérique est un accélérateur de démocratie. Je pense qu'effectivement, quand nous observons le contexte dans lequel les partis pirates se sont créés, la ferveur de leur naissance, l'adhésion populaire massive... par la suite, nous avons l'impression qu'ils ont une difficulté à s'imposer dans le temps. On peut donc se poser la question de savoir si ce n'est pas le mode opératoire même de ces partis qui pose problème. Ces derniers naissent d'une contestation mais ils n'ont aucun programme de gouvernement, aucun projet de société, alors qu'il s'agit des critères essentiels des partis politiques. J'essaye de faire un parallèle avec Podemos et le Mouvement 5 étoiles, qui se définissent comme des mouvements citoyens mais qui sont finalement des partis politiques de circonstance, sans un contexte précis, ils n'auraient pas vu le jour. Ces partis peuvent-ils constituer une sorte de menace pour les partis politiques traditionnels? Il y a deux mouvements citoyens en Afrique : le mouvement «Y'en a marre » au Sénégal et «Allez citoyen» au Burkina Faso qui sont des mouvements de contestation qui sont nés des réseaux sociaux mais qui n'ont pas de vocation politique, ils ont un rôle démocratique dans le pays, cela suscite un vrai intérêt sur les réseaux sociaux: ce sont des sentinelles de la démocratie. 
Michaël Bardin: Il y a une première et fondamentale distinction à faire entre «mouvement citoyen » et «parti politique » : il y a ceux qui se présentent à des élections et qui sont élus et il y a ceux qui en restent aux manifestations. J'ai structuré mon propos autour du Parti pirate, de Podemos et du Mouvement 5 étoiles, parce qu'eux, à un moment donné, s'enregistrent en tant que partis politiques, participent à des élections avec des résultats plus ou moins bons mais avec des résultats; c'est-à-dire qu'ils siègent aujourd'hui dans des institutions politiques. Si je distingue le Parti pirate de Podemos et du Mouvement 5 étoiles, c'est pour des questions de fonctionnement interne. Dire que le parti pirate n'a pas de programme politique, c'est faux, ils ont un programme très complet. Le problème de ce dernier est le logiciel qui a servi à son élaboration, et qui, par ailleurs, est le même qui a servi à fédérer le parti. Liquidfeedback permet à tous les adhérents de faire des propositions. Quand elles sont soutenues par un certain nombre de pirates, commence alors un débat: la proposition est étudiée, discutée, amendée et finalement votée. Sur cette base, et sur la multiplication des propositions, un programme politique est élaboré et structuré. Mais le contenu reste pauvre en propositions précises : tout le monde a droit à une «bonne santé», tout le monde a droit à «l'accès à un médecin», tout le monde a le droit «d'accéder à la culture », etc. C'est le propre de l'ouverture à l'extrême. C'est une forme de compromis à l'extrême. Plus il y a d'ouverture, plus il y a d'avis, plus le compromis est large. Concernant Podemos et le Mouvement 5 étoiles, je serais tenté de dire qu'il n'y a pas de programme. Dans le cas de Podemos, le programme en 394 points est tellement vaste qu'il devient une «litanie» dont on ne voit jamais la fin, avec des propositions qui ne sont pas toujours cohérentes entre elles. Quant au Mouvement 5 étoiles, et même s'il emprunte aujourd'hui la voie de la plateforme collaborative avec Rousseau, le programme, si cela peut être ainsi nommé, reste aussi abscons sur sa direction politique que sur les méthodes qui conduisent à l'élaborer. Je reste persuadé que le Mouvement 5 étoiles est la «version numérique» de la Ligue du Nord des années 1990-2000: un mouvement ultra-centralisé qui bénéficie d'un rejet des partis traditionnels, un rejet consciencieusement entretenu par une «tête de gondole» très médiatique. L'exercice du pouvoir risque de poser problème aux Grillini, et c'est même déjà le cas. Pour Podemos, le vent a déjà commencé à tourner, parce que le pays est en train de changer, que les équilibres politiques changent. La position du secrétaire général va devenir de plus en plus délicate. Unidos Podemos permet pour l'instant d'éviter une alliance avec le PSOE mais des rapprochements devront s'opérer si le parti veut réellement jouer un rôle dans la vie politique espagnole. Et là, les choses vont devenir intéressantes, puisqu'il va falloir passer le cap des revendications pures, faire le tri dans tout ce qu'ils ont soutenu jusqu'à maintenant. Il y aura forcément un tri pour trouver des accords, même ponctuels, avec le PSOE. Sur le reste, les mouvements citoyens, au sens large, sont nombreux - et utiles - mais ils «influencent» éventuellement le pouvoir, ils ne l'exercent pas, à l'inverse de ces nouveaux partis.

Public: Je voulais dire que la vocation des mouvements citoyens, au départ et comme pour Podemos, c'est la contestation.

Michaël Bardin :Certes, mais Podemos n'est pas un mouvement de contestation, c'est la retranscription en parti politique de nombreux mouvements de contestation. Un cap est déjà franchi avec la 
création Podemos qui n'est qu'un parti politique et constitue justement l'évolution du Mouvement des indignés mais également de plus d'une vingtaine de mouvements citoyens. À partir du moment où c'est un parti politique, en tant que tel, on attend d'eux autre chose que de la contestation.

Public: Par rapport aux réseaux sociaux comme catalyseurs de l'action politique surtout dans les campagnes, j'ai pu observer un cas très particulier récemment au Niger. Un des candidats s'est retrouvé en prison mais grâce à l'utilisation massive des réseaux sociaux, il a pu se placer au deuxième tour des élections. Son équipe de campagne a pu agir sur le terrain grâce aux réseaux. Cette utilisation des réseaux sociaux est en train de devenir un catalyseur de démocratie dans le contexte politique africain, cela représente donc une forme d'aubaine pour les hommes politiques durant la campagne.

Public (Caterina Severino): Je voudrais revenir sur la question des partis pirates et du Mouvement 5 étoiles pour faire une remarque concernant les rapports avec les partis traditionnels: le Mouvement 5 étoiles s'est structuré sur internet, ce sont des enfants essentiellement d'internet, et le parti voulait vraiment faire la distinction entre le mouvement et les partis traditionnels. Il y avait ce côté critique à l'égard des partis traditionnels. Mais on remarque qu'après être devenu la troisième force de l'Italie, et maintenant qu'ils ont pris des villes comme Rome, au moment où ils commencent à avoir du pouvoir, ils fonctionnent comme des partis traditionnels. Ils disaient au départ qu'il fallait éviter les médias car, selon eux, ces derniers étaient corrompus (connivence avec le pouvoir en place), mais désormais on ne les voit que dans les médias traditionnels : télévision, journaux, radios. Finalement on retrouve le même fonctionnement que dans les partis traditionnels.

Michaël Bardin : Ils ont cultivé toute la distance qu'ils ont pu aussi longtemps que cela a été possible, en revendiquant comme base fondamentale: «nous ne sommes pas comme les autres, nous ne sommes pas un parti». L'un des 8 articles du statut prévoit bien que toute la communication des élus du Mouvement 5 étoiles est maîtrisée par les dirigeants nationaux. Cela dit, avec un nombre d'élus aussi important, la réalité pratique est désormais tout autre et oui, effectivement, il y a un vrai changement de cap dans la communication du parti.

Public (Caterina Severino): Sur le fonctionnement intérieur de ce Mouvement 5 étoiles : l'idée est une démocratie plus complète s'appuyant sur la société civile qui, selon les termes mêmes du parti, «vient du bas». Finalement, si l'on regarde le fonctionnement interne du parti, on remarque qu'il y a une forme de totalitarisme, de parti autoritaire. Les personnes qui ne disent pas ce qu'il faudrait dire ont été exclues, des élus locaux ont ainsi été évincés du parti. Le dirigeant, l'un des cofondateurs du Mouvement, avait par ailleurs une entreprise qui était ancrée dans le numérique. La direction est donc très forte et il existe des élites au sein même du parti. C'est donc l'inverse de ce qu'ils prônent, de ce qu'ils visent.

Michaël Bardin: Après la victoire aux législatives, Beppe Grillo, la vraie figure de proue du mouvement a dit qu'il était fatigué et qu'il se retirait. Il y a donc eu un directoire de six parlementaires qui a été mis en place. Il n'y a jamais eu autant de démissions au sein du Mouvement 5 étoiles. 
Tant et si bien que quelques mois plus tard, le directoire a littéralement disparu des médias et Beppe Grillo a repris la tête du mouvement et a recommencé à aller dans les médias. Étrangement, tout a recommencé à mieux fonctionner.

Public (Caterina Severino): Ce sont les désillusions du numérique comme nous en avons parlé un peu plus tôt.

Président (Pascal Jan): Là on est sur le fonctionnement interne des partis, on s'échappe un peu de la démocratie connectée. Simplement, on peut faire élire un président contre un parti, avec l'exemple du président américain. Ce matin a été évoquée la crise de la représentation, ce ne sont pas que les élus, ce sont les formations traditionnelles politiques. Le fait que Donald Trump soit élu, contre les élites de son parti. Tout a été fait pour noyauter complètement Donald Trump et cela a échoué, il a résisté en dehors du parti. Ce qui est relativement intéressant, d'autant plus que la campagne de Donald Trump était en partie contre les médias. La puissance médiatique n'est pas forcément égale à la puissance numérique. Cette dernière donne l'impression que l'on peut maîtriser, que l'on peut participer, alors que la puissance médiatique est imposée. Il n'y a pas la même réaction face au numérique, en comparaison avec la puissance médiatique, ici, il est possible d'être acteur. C'est un autre débat mais la question doit aussi être pensée. La puissance des médias dans les élections a toujours été évoquée mais elle n'est pas de la même nature que la puissance numérique.

Public: Ce qui me frappe, c'est la culture de la dérision qui inonde les médias et les réseaux sociaux. Il y a peu de temps, j'étais dans un pays d'Amérique centrale où j'ai eu droit à quinze chaînes de sports, dix chaînes pour enfants, cinquante chaînes de télénovelas, une chaîne évangéliste, une chaîne catholique, une chaîne du Costa Rica. Où sont les chaînes qui peuvent élever les esprits? Beppe Grillo est bien un comique au départ. Trump a participé à des émissions où le rire était utilisé. Est-ce qu'on ne va pas se poser des questions sur cette culture du divertissement et de la dérision? On assiste à un aplatissement du monde et un manque flagrant de culture générale que l'on remarque chez de nombreux étudiants, c'est-à-dire l'oubli du contexte spatial et temporel.

Public: Pour nuancer ce qui a été dit sur l'influence des médias, elle a été décisive. Ils n'ont pas influencé dans le sens qu'ils auraient souhaité mais ils ont été décisifs dans le sens où ils ont fait la publicité de Donald Trump. J'aimerais savoir ce qu'il en aurait été s'il y avait eu un black-out, estce que cela aurait été la même chose? Il en va de même pour les partis d'extrême-droite, comme en France. Sans relais médiatiques je pense que beaucoup de choses tomberaient à l'eau. En plus, Donald Trump est un candidat contre le système et les médias représentent le système, les médias sont contre lui, donc cela nourrit le système finalement.

Idris Fassassi : La question c'est la légitimité d'un éventuel black-out justement, comment justifier que les médias prennent parti pour un candidat plutôt qu'un autre en l'écartant totalement du débat? 
Public: Ils prennent déjà parti. Ils sont contre. Alors quelle légitimité aussi à être contre? Chacun son choix. C'est aussi un parti pris. Être contre c'est aussi le même parti pris que «je ne l'invite pas », c'est un choix.

Idris Fassassi: C'est vrai, mais je pense qu'il y a toutefois une différence entre combattre les idées d'un candidat, et l'écarter totalement en ne relatant aucun des éléments de sa campagne. C'est beaucoup plus fort, plus violent et pose un réel problème. Cela aurait peut-être radicalisé davantage la campagne de Donald Trump, et renforcé sa posture de candidat hors système, qu'il n'est d'ailleurs pas en réalité.

Public: Quel est le pourcentage des personnes populaires qui utilisent les réseaux sociaux?

Idris Fassassi : Près des deux tiers des Américains sont sur les réseaux sociaux, plus de 200 millions de personnes.

Public: Les ouvriers savent aussi se servir d'internet...

Public: Bien sûr, ce n'est pas la question de «savoir » mais de ce qu'il en est effectivement en pratique. Bien sûr, tout le monde peut se servir d'internet même ma grand-mère pourrait se servir d'internet... pourtant elle ne l'a pas! (Rires)

Public: Je partage le même pessimisme, il y a une expression qui est revenue souvent dans les interventions : il faudrait «transformer l'essai ». Je pense que la démocratie connectée est condamnée à rester connectée. Peut-elle avoir une traduction dans l'espace réel et démocratique dans lequel nous vivons? Je voudrais poser une question par rapport aux États-Unis, 200 millions d'utilisateurs Facebook, une campagne très connectée, 500 millions de budget investis : vous avez parlé d'un triptyque (information, contribution, mobilisation), personnellement je vérifie surtout les deux premiers : j'utilise les réseaux sociaux pour m'informer, je participe aussi au débat dans mon pays, par contre le troisième élément... Souvent, j'ai «mieux à faire» que de manifester. Je demande si dans une élection majeure où l'on comptabilise presque cinquante pour cent d'abstention, les réseaux sociaux n'ont-ils pas échoué dans la mobilisation ou au contraire cela aurait pu être pire? Je pense que le problème est là: de passer d'un espace connecté à un espace réel.

Idris Fassassi: Je suis plutôt sceptique quant au rapport entre les citoyens et le candidat, quant à l'établissement d'un dialogue et d'une véritable «connexion» Je pense que c'est plus la logique unidirectionnelle, du candidat vers les citoyens, qui est effective. Pour le candidat cela fonctionne très bien. Voyez Donald Trump! Il a pu, dans une large mesure, court-circuiter les médias classiques et atteindre des couches particulières de l'électorat. Les réseaux sociaux sont effectivement un formidable outil de campagne. Maintenant, vouloir reconstruire une nouvelle démocratie sur les réseaux sociaux, je serais plus réservé.

Président (Pascal Jan): Je vous remercie pour l'organisation de ce beau colloque. 


\title{
Propos conclusifs \\ QUEL AVENIR POUR LE CITOYEN DANS LA « DÉMOCRATIE NUMÉRIQUE »?
}

\author{
Marthe Fatin-Rouge Stefanini ${ }^{1}$
}

Cette journée d'étude sur la démocratie connectée a été particulièrement riche. De nombreuses questions ont été abordées et ont conduit à réfléchir sur le fonctionnement des démocraties aujourd'hui au regard des évolutions et des potentialités offertes par les nouvelles technologies et les nouveaux vecteurs de communication. Le thème étudié est, en effet, au cœur des préoccupations de notre société ces dernières années et ces préoccupations sont d'autant plus nombreuses que l'on découvre l'emprise qu'internet et les réseaux sociaux ont déjà, ou sont susceptibles d'avoir, sur la vie quotidienne et le fonctionnement des institutions. Ce thème intéresse d'ailleurs tout un chacun mais génère un sentiment ambigu mêlant à la fois l'admiration et l'effroi. Chaque individu a sa propre opinion, ses propres exemples ou anecdotes tirés d'informations lues, entendues, tweetées, diffusées de quelque manière que ce soit et quelle qu'en soit la véracité...

Les interventions et les débats ont porté notamment sur les enjeux, les réalités et les faux-semblants de la démocratie numérique. Il convenait en particulier de se demander si le développement d'internet, et les interactions qu'il permet, participent au renouveau de la démocratie dont on ne cesse de constater qu'elle est en crise; une crise de la représentation mettant en péril les démocraties même les plus anciennes. Internet pourrait ainsi offrir la promesse d'un « renouveau de la démocratie », d'une « cure de jouvence démocratique » comme l'a souligné Ariane Vidal-Naquet, permettant aux citoyens non seulement de participer mais également de devenir « co-legislateur, œuvrant avec, aux côtés du législateur traditionnel ».

En effet, la démocratie à l'ère d'internet a ouvert la porte à de grandes ambitions en ce qu'elle révolutionne le rapport des citoyens - et, au-delà, des individus - à la politique, au sens large du terme, pour se rapprocher de l'idéal démocratique de base, permettre à chacun de participer plus ou moins directement à la prise de décision politique. Grâce à la démocratie « connectée », la taille de l'agora s'est ainsi considérablement élargie. Chaque personne pour peu qu'elle veuille bien être pleinement « connectée » à la société, se sent directement concernée puisqu'elle est une des voix de cette démocratie. Le numérique lui offre l'occasion de participer directement au débat démocratique

1 Directrice de recherches au CNRS, Aix-Marseille Univ., Université de Toulon, Univ. Pau \& Pays Adour, CNRS, DICE, ILF-GERJC, Aix-enProvence, France. 
sans qu'il soit nécessaire d'y être préalablement invité. Ainsi, ce n'est pas seulement chaque voix qui compte, mais chaque personne, chaque message, chaque opinion, d'où qu'ils proviennent. Chaque internaute, en particulier ceux qui participent à des blogs ou des forums de discussions, a de cette façon le sentiment de devenir acteur du débat démocratique ${ }^{2}$. Il en va de même pour ceux qui envoient des sms qui sont diffusés au cours d'émissions télévisées, afin de participer activement aux débats organisés dans celles-ci. La démocratie représentative serait ainsi « idéalisée », selon les termes de Priscilla Jensel-Monge, en offrant la possibilité d'une démocratie plus directe car « connectée ». En outre, bien que des filtres existent encore concernant ces différentes formes de participation, ils se révèlent beaucoup plus souples de sorte qu'il ne soit pas nécessaire d'être journaliste, homme politique, philosophe réputé, universitaire ou chercheur pour prendre part au débat. On assiste ainsi - et par là même - à une désacralisation de la parole politique car ce qui importe désormais est plus le message énoncé que le statut de celui qui l'énonce.

Le numérique permet également un accès facilité à l'information, une « démocratisation des savoirs $»^{3}$ par un accès plus simple à la connaissance. Pour en revenir au seul droit constitutionnel, le numérique a d'ailleurs largement facilité l'essor du constitutionnalisme non seulement en théorie, dans les constitutions, par la diffusion du constitutionnalisme et l'accès facilité aux jurisprudences et à la doctrine étrangères mais également dans les faits. Ainsi, internet n'a pas permis seulement la diffusion de modèles théoriques, d'articles de la Constitution, de mécanismes institutionnels et de solutions jurisprudentielles; il a contribué également à la diffusion du phénomène démocratique en soi, avec des revendications politiques s'appuyant sur une possibilité de dénoncer par l'image, et par internet, ce qui se fait dans les États, notamment les répressions dans la violence ${ }^{4}$. D’ailleurs, cette capacité de pression sur les régimes autoritaires par le biais des réseaux sociaux en particulier, est contrebalancée par la volonté de ces régimes, de réduire, interdire, contrôler l'accès à internet ou d'en contrôler le contenu. La circulation de l'information est donc à double tranchant car elle peut nuire y compris à celui qui procède à la diffusion. Ainsi, dans les régimes autoritaires ou les démocraties fragiles ou fragilisées, chacun est sous surveillance.

En revanche, dans les démocraties plus solides, du point de vue du citoyen en particulier, les potentialités offertes par le numérique sont particulièrement attrayantes même si ce pouvoir de participation des individus n'est peut-être qu'illusion ${ }^{5}$ comme le rappellent Idris Fassassi et Priscilla JenselMonge. D’ailleurs, au fur et à mesure que les nouvelles technologies se développent et prennent une place de plus en plus importante dans la vie quotidienne et dans la société en général, on en perçoit aussi les inconvénients. Or, sans vouloir être pessimiste ou conservateur à l'excès face aux opportunités offertes par la toile, force est de constater que lors de cette journée d'étude les interventions et les discussions ont principalement porté sur les défauts et les dangers du numérique pour la

\footnotetext{
2 Voir sur ce point M. Fraysse, «La promotion de l'opinion individuelle : les blogs », in Ph. SÉGUR, S. PÉRIÉ-Frey, L’internet \& la démocratie numérique, Presse universitaires de Perpignan, 2016, 219 p., disponible en open edition: http://books.openedition.org/pupvd/2748 (consulté le 8 février 2018)

Voir M. Delmas-Marty, « La démocratisation des savoirs », Rue Descartes, 2007/1 (nº 55), p. 128.

Voir M. FraysSE, précité, §18.

Voir en ce sens, G. VIDAL, « Présentation : Instabilité et permanence des usages du numérique », Les cahiers du numérique, vol. 9 (2), 2013 , p. 9-46 (en ligne sur Cairn: www.cairn.info/revue-les-cahiers-du-numerique-2013-2.htm).
} 
démocratie. Si le numérique ouvre de formidables perspectives en termes de participation et d'accès facilité aux informations, la connexion numérique, la mise en réseau et la circulation d'une quantité d'information présentent, toutefois, un certain nombre de défauts qui ont été évoqués tout au long de cette journée, et dont la liste n'est pas exhaustive:

- la « déprofessionnalisation de l'information $»^{6}$, pour commencer, chacun s'érigeant en journaliste, à travers des blogs, des photographies, et tout type d'information en libre accès sur internet sans que cette diffusion soit soumise à un minimum de règles déontologiques;

- une absence de hiérarchisation des informations : la moindre anecdote peut avoir un fort retentissement si elle est largement diffusée. En outre, si l'accès à la connaissance est plus direct, il n'est pas forcément plus pratique car il est nécessaire d'être capable de sélectionner les bonnes informations, celles qui sont pertinentes pour celui qui fait une recherche, face à une masse très importante de données;

- un risque de désinformation voire de manipulation des informations ${ }^{7}$ comme le soulignent Idris Fassassi et François-Bernard Huyghe: les « fake-news » (fausses informations) ou « réalités alternatives » sont d'ailleurs source d'inquiétudes pour les gouvernants au regard de l'effet dévastateur que cela peut avoir sur le fonctionnement même de la démocratie; d'autant plus lorsque ces fausses informations sont relayées par des médias officiels eux-mêmes victimes de ce nouveau mode de diffusion anarchique.

- dans la campagne politique, un risque d'abus du marketing politique par une utilisation des sites internet et des réseaux sociaux à des fins de remporter les élections (Michaël Bardin) au détriment de la sincérité et de la loyauté d'une campagne électorale qui devraient être de mises dans une démocratie;

- un appauvrissement du débat politique, sous l'effet d'un nivellement par le bas, voire une « négation du débat » comme le rappellent Idris Fassassi et Priscilla Jensel-Monge, la discussion entre représentant et représenté prenant la forme d'un monologue 8 .

D’autres éléments ont également été portés au débat. D’un côté, le web permet un accès facilité au débat politique voire à la participation à la prise de décision politique et, en théorie, on tend à s'approcher de l'idéal démocratique. Cependant, d'un autre côté, ce mode de participation souffre d'un manque de transparence, donc de visibilité des participants dont il est difficile d'établir l'identité réelle. La démocratie numérique peut donner l'illusion d'une participation générale là où seuls quelques citoyens, certains citoyens - disposant de temps ou pour qui le sujet revêt un intérêt capital (intérêt pouvant être personnel) -, ont contribué à la fabrication de la norme. En outre, la

\footnotetext{
M. Fraysse, précité, § 22.

En ce sens, voir notamment, V. EsPIAGo, « Désinformation, subversion, hacking : les actions anti-démocratiques », in Ph. SÉGUR, S. PÉRIÉFreY, L'internet \& la démocratie numérique, op. cit.

8 Voir en ce sens, Ch. Euzet, «Internet, Numérique: Quel horizon pour la démocratie », in Ph. SÉGUR, S. PÉRIÉ-FrEY, L’internet \& la démocratie numérique, op. cit., §35.
} 
participation démocratique connectée n'a pas de frontière et même sur un projet national ou local, n'importe quel internaute peut interagir, influencer le débat voire la co-rédaction d'un texte. Ainsi, Ariane Vidal-Naquet de se demander, à propos de la Loi Lemaire, si « la loi connectée peut encore être considérée comme l'expression de la volonté générale ». Sans compter que des prodiges de l'informatique peuvent donner beaucoup de poids à un phénomène ou une action provenant d'une minorité, tout simplement parce qu'ils savent très bien exploiter toutes les opportunités que leur offre ce mode de communication. Ainsi, il existe un risque de manipulation par la création artificielle de mouvements de foule à des fins strictement subversives (astroturfing ${ }^{9}$, hacking) ou plus largement, par l'influence de mouvements ou de groupes dont les objectifs ne sont pas démocratiques.

En outre, la participation au débat politique, au sens large du terme, par le biais de l'internet, si elle permet à de nouveaux mouvements d'émerger (Mouvement Cinq Étoiles en Italie, Podemos en Espagne...), est aussi susceptible de favoriser une démocratie de défiance ${ }^{10}$, une démocratie contestataire et non constructive, certains mouvements n'ayant pas d'autre programme politique que la contestation comme l'indique Michaël Bardin.

Dans le même ordre d'idée, la démocratie «connectée » offre la possibilité de contrôler davantage les gouvernants et peut encourager ces derniers à être plus performants comme l'a rappelé Jean-Philippe Derosier. Elle s'est accompagnée d'une plus grande transparence des données publiques et de la vie politique à la demande des citoyens eux-mêmes. Toutefois, non seulement cette 《sous-veillance $»^{11}$ (ou « vigilance par la base ») permanente fait peser une forte pression sur les gouvernants mais risque également, comme l'a souligné Pascal Jan, de contribuer à la désacralisation de la fonction de représentation; ce qui, plus largement, pourrait affaiblir encore la volonté des citoyens de s'engager en politique. De même, comme l'a indiqué Priscilla Jensel-Monge, les réseaux sociaux semblent réduire la distance entre les gouvernants et les gouvernés par un accès facilité aux gouvernants et donnent une impression de proximité. Cependant, la pratique semble révéler que cela n'est qu'une apparence, une illusion de proximité qui risque de se traduire, dans quelque temps, par une recrudescence de la perte de confiance des citoyens envers la classe politique.

La connexion rapide et permanente produit également une forme de pression sur les gouvernants pour décider, pour réformer. Cependant, cette pression par la communication n'est pas favorable au temps de la réflexion, ni à la distance nécessaire par rapport à l'objet et au contenu de la réforme demandée. À l'inverse, une réaction trop rapide dès l'annonce d'une réflexion sur une éventuelle réforme, risque d'avoir un effet dissuasif par crainte d'une réaction trop forte de l'opinion publique, particulièrement à l'approche d'échéances électorales.

9 «Astroturfing »: «stratégie de communication dont la source réelle est occultée et qui prétend à tort être d'origine citoyenne », S. Boulay, «Exploration du phénomène d'astroturfing: une stratégie de communication usurpant l'identité citoyenne dans l'espace public », Communiquer [En ligne], 7 | 2012, mis en ligne le 21 avril 2015, consulté le 8 février 2018. URL: http://journals.openedition.org/ communiquer/487; DOI: 10.4000/communiquer.487.

10 Internet favorise ainsi la «Contre-démocratie » analysée par Pierre Rosanvallon (La contre-démocratie. La politique à l'âge de la défiance, Seuil, 2006, 345 p.)

11 Expression de Steve Mann, voir S. Mann, J. Nolan, B. Wellman, « Sousveillance: Inventing and Using Wearable Computing Devices for Data Collection in Surveillance Environments », Surveillance \& Society, 2003, www.surveillance-and-society.org, (consulté le 19 février 2018). 
Ce qu'il ressort des débats de cette journée, en définitive, est que pour l'instant la démocratie numérique n'a pas vocation à remplacer la démocratie représentative car même si la démocratie représentative n'est pas parfaite, même si elle est en crise, la démocratie numérique est très imparfaite, elle n'est pas suffisamment fiable. Elle est encore à un stade infantile, elle ne fait pas système, elle ne peut pas être un modèle de gouvernement. Ce constat n'est pas étonnant car même en Suisse, où des instruments démocratiques tels que l'initiative populaire et le référendum sont très présents, offrant à la « base » une possibilité bien plus accrue qu'en France de prendre part et de peser sur le débat politique, ces outils complètent mais ne se substituent pas à la démocratie représentative car la participation des citoyens, que ce soit de manière directe ou par voie numérique, ne peut être efficace sans une forme d'organisation, en termes de procédures et de contrôles notamment, que propose la démocratie représentative. Cette dernière constitue, jusqu'à présent, le meilleur moyen de relayer la volonté des citoyens et de prendre des décisions au plus près des préoccupations de ceux-ci. La démocratie numérique peut d'autant moins faire système qu'elle n'est pas définissable, on ne peut en cerner les contours. La démocratie, par définition, connaît le désordre, et le numérique amplifie ce désordre. Ce côté non maîtrisable, le risque de déstabilisation que génère la participation à la démocratie par le numérique est particulièrement dérangeant pour les gouvernants mais également pour les médias traditionnels et la manière dont fonctionnent habituellement les démocraties. La campagne électorale du Président des États-Unis, en 2016, a montré non seulement que les médias étaient complètement dépassés par les informations diffusées sur les réseaux sociaux mais également qu'ils ont pu être instrumentalisés par les candidats.

$\mathrm{Au}$ regard du fait que les démocraties ne peuvent échapper à la révolution numérique, une réflexion semble donc nécessaire sur le point de savoir comment, dans ce contexte, préserver les valeurs démocratiques et en particulier favoriser l'intégrité de la démocratie. Cette préservation, me semble-t-il, suppose un effort d'adaptation permanente de la démocratie à l'outil numérique. Cela passe par une forme d'éducation au numérique par un développement de l'esprit critique, une attention particulière portée à l'éducation des citoyens, des candidats, des élus et, plus largement, de l'ensemble des individus à la participation sur internet. Il reste cependant à espérer que cette phase transitoire dans laquelle les démocraties se situent, en ce moment, et qui doit permettre une adaptation au numérique, ne soit pas permanente tant l'évolution des nouvelles technologies et des changements qu'elles proposent est rapide. 

TABLE DES MATIÈ RES

\section{Propos introductifs}

Ambitions et limites de la démocratie connectée 9

Caterina SEverino

\section{Partie I}

Citoyens et NumériQue : DE LA COMMUNiCATION PAR INTERNET À L'IDÉE D'UNE RÉPUBLIQUE NUMÉRIQUE

Propos introductifs

Jean GicQUeL

LE CITOYEN CO-LÉgislateur : QUAND, COMMENT, POUR QUELS RÉSULTATS?

Ariane VIDAL-NAQUET

I. Quand ? Le foisonnement des expériences de co-législation citoyenne

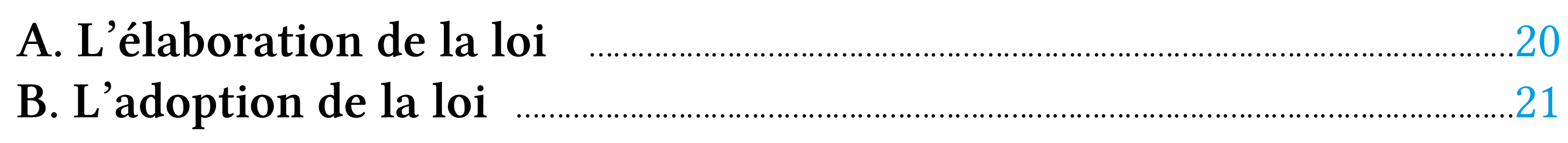

II. Comment ? Le fonctionnement de la co-législation citoyenne ....22

A. Les acteurs de la co-législation

B. Les modalités de la co-législation 
III. Pour quels résultats? Le renouvellement de la démocratie représentative?

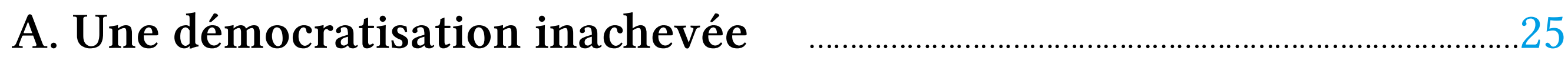

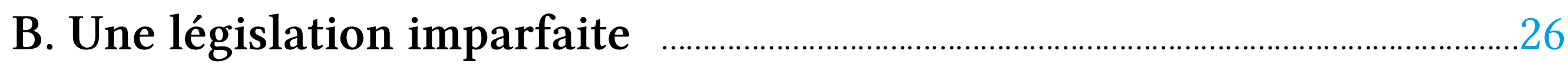

LE NUMÉ RIQUE COMME OUTIL DE CONTRÔLE DES GOUVERNANTS _.....29 Jean-Philippe Derosier

I. S'ouvrir modérément au contrôle

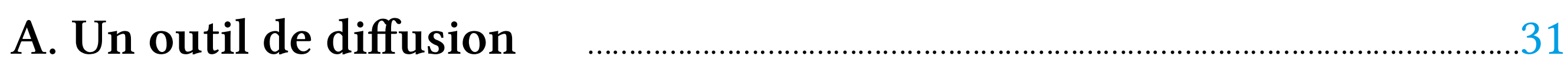

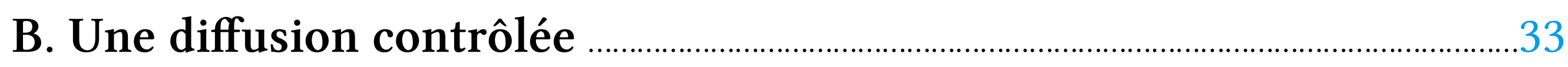

II. Subir assurément le contrôle …………............................................................................

A. Un outil d'enquête

B. Un contrôle relayé

Discussion -

GARANTIES DÉMOCRATIQUES ET NUMÉRIQUE _................................................49

Sophie LAMOUROUX

I. Droit des élections et numérique : une réglementation adaptée $\quad \ldots . . .52$

II. Contentieux électoral et numérique : une sincérité du scrutin à préserver

NumériQue : ACTIVISMe et influence PolitiQue

François-Bernard HuYghe

Discussion 


\section{Partie II}

GOUVERNANTS ET NUMÉRIQUE : ENTRE VÉRITABLE OPPORTUNITÉ ET RISQUE D'INSTRUMENTALISATION

\section{Propos introductifs}

Pascal JaN

LES PARTIS POLITIQUES FACE AU NUMÉ RIQUE : ENTRE ADAPTATION STRATÉGIQUE ET ÉMERGENCE DE NOUVEAUX MOUVEMENTS

I. L'émergence de nouveaux mouvements

A. L'antipartisme organisé... en parti politique

B. Le citoyen comme garant du renouveau de la classe politique

II. Les nouveaux partis et la difficile conciliation entre transparence et efficacité

A. Les risques d'un « centralisme cybercratique » .................................................85

B. Un impact politique d'une durée incertaine ...................................................87

1. Un apport à la démocratie encore incertain ......................................................87

2. Une capacité à perdurer difficile à évaluer .........................................................89

III. Numérique et campagnes électorales

A. Le numérique comme générateur de particularismes ?

B. Le numérique comme instrument de rationalisation

1. De la rationalisation de la campagne au profilage électoral

2. Du profilage électoral au fichage des électeurs et des adhérents 
L'UTILISATION DES RÉSEAUX SOCIAUX PAR LES PARLEMENTAIRES : INSTRUMENT DE REVITALISATION DE LA DÉMOCRATIE RE PRÉ SE NT ATIVE?

Priscilla Jensed-Monge

I. Un dépassement des limites de la démocratie représentative

A. La permanence de la démocratie

B. La poursuite de l'idéal délibératif

II. Une accentuation des défauts de la démocratie représentative $\quad . .106$

A. La fiction délibérative

B. Le risque de renforcement de la crise de confiance politique

LES EffETS DES RÉSEAUX SOCIAUX DANS LES CAMPAGNES ÉLECTORALES AMÉ RICAINES

Idris FASSASSI

I. Les réseaux sociaux en tant que catalyseur de la mobilisation et de l'action politiques

A. Un vecteur de l'engagement politique pour les citoyens

B. Une arme de campagne redoutable pour les candidats

II. L'usage des réseaux sociaux en tant qu'obstacle à la délibération politique

A. L'appauvrissement du débat

B. La négation du débat

Discussion

\section{Propos conclusifs}

QUEL AVENIR POUR LE CITOYEN DANS LA « DÉMOCRATIE NUMÉRIQUE »?

Marthe Fatin-Rouge Stefanini 
UMR Droits International, Comparé et Européen (DICE)

Espace René Cassin

3 , avenue Robert Schuman

13628 Aix-en-Provence

dice-editions@univ-amu.fr

Composition et mise en page :

Donia Landoulsi, UMR DICE, Aix-Marseille Université

Conception de la couverture :

Donia Landoulsi, UMR DICE, Aix-Marseille Université

Illustration de la couverture :

Creative Commons CCO

$3^{\text {e }}$ trimestre 2018 